\title{
Spacecraft Formation Guidance and Control on J2-Perturbed Eccentric Orbits
}

\author{
by \\ Bradley Kuiack

\section{Master of Applied Science in Aerospace Engineering} \\ Carleton University \\ Ottawa, Ontario \\ (C) 2018 \\ Bradley Kuiack
}

A thesis submitted to the Faculty of Graduate and Postdoctoral Affairs in partial fulfillment of the requirements for the degree of 
This work is dedicated to my late grandfather, Wasyl Wasko. 


\begin{abstract}
Spacecraft formation flying is currently an important and thriving area of research, with plenty of unique challenges to overcome in the field of spacecraft dynamics, and a wide variety of useful applications if these challenges may be overcome. The guidance of the relative motion of a spacecraft formation is an area of particular interest. Conventional space missions typically require a guidance system which outputs the desired orbit of a single spacecraft. However, in a formation, the position of one spacecraft relative to another is of greater interest, and thus the guidance system must handle the more difficult task of determining the desired relative motion of the formation.

Many models for spacecraft formation flying exist, but all have their limitations, either in accuracy due to assumptions made in their derivation like using circular orbits or neglecting important orbit perturbations, or in computational efficiency by requiring numerical integration. This thesis presents a novel set of analytical equations of motion describing the position of a follower spacecraft relative to a leader spacecraft in formation which is valid for eccentric orbits and which incorporates the so-called $J_{2}$ perturbation. This set of three equations, one for each priniciple direction in the local-vertical-local-horizontal reference frame, is then validated for accuracy by comparison with a numerical simulator. Finally, the equations are verified in a number of practical applications, focussing on their use as a guidance system in spacecraft formation guidance and control simulations.
\end{abstract}




\section{Acknowledgements}

First and foremost I would like to thank my supervisor Steve Ulrich for giving me the opportunity to pursue this work, for his support and encouragement along the way, and most of all for his patience, understanding and kindness throughout this project. Certainly none of this would have been possible without his help. I would also like to thank my family who are all always there for me, especially my parents, John and Lorna Kuiack for their love, and unending support. Finally I would like to thank Giuliana Velarde for helping me stay motivated and on track, and for her many hours spent editing my work.

This research was financially supported by the Natural Sciences and Engineering Research Council of Canada through both the Undergraduate Student Research Award program, and via the Canada Graduate Scholarship. Additionally, Carleton University and the Department of Mechanical and Aerospace Engineering helped fund this research through an entrance scholarship and Teaching Assistantships. 


\section{Table of Contents}

Abstract $\quad$ iii

Acknowledgements $\quad$ iv

List of Tables viii

List of Figures $\quad$ ix

List of Symbols $\quad$ xiii

List of Acronyms xvi

$\begin{array}{lll}\text { Chapter } 1 & \text { Introduction } & 1\end{array}$

1.1 Motivation ...................... 1

1.2 Problem Statement . . . . . . . . . . . . . . . . . . . 1

1.3 Previous Work . . . . . . . . . . . . . . . . . . . . 3

1.4 Thesis Objectives . . . . . . . . . . . . . . . 5

1.5 Contributions ........................ 5

1.6 Organization ......................... 6

$\begin{array}{lll}\text { Chapter } 2 & \text { Basic Concepts } & 8\end{array}$

2.1 Introduction . . . . . . . . . . . . . . . . . . . 8

2.2 Reference Frames . . . . . . . . . . . . . . . . . . . . 9

2.3 Spacecraft Motion on Keplerian Orbits . . . . . . . . . . . . . . . . . 10

2.4 Orbital Elements . . . . . . . . . . . . . . . . . . . 13

2.4.1 Conversion from Inertial Position and Velocity to Orbital Elements . . . . . . . . . . . . . . . . . 14

2.4.2 Conversion from Orbital Elements to Inertial Position and Velocity ........................... 18

2.4.3 Anomaly Values . . . . . . . . . . . . . . . . . . . . . . 18 
2.5 Rotation Matrices . . . . . . . . . . . . . . . . . . . . 20

2.6 Orbital Perturbations . . . . . . . . . . . . . . . . . . . . . . . . 21

2.7 Numerical Simulator . . . . . . . . . . . . . . . . . . . . 23

2.8 Hill-Clohessy-Wiltshire Model for Formation Flying . . . . . . . . . 24

$\begin{array}{lll}\text { Chapter } 3 & \text { Proposed Equations of Motion } & 27\end{array}$

3.1 Introduction . . . . . . . . . . . . . . . . . . . 27

3.2 Derivation of Equations of Motion . . . . . . . . . . . . . 27

3.2.1 Relative Motion on Keplerian Eccentric Orbits . . . . . . . . 27

3.2.2 Relative Motion on $J_{2}$-Perturbed Eccentric Orbits . . . . . 29

3.2.3 Simplification of Proposed Equations . . . . . . . . . . . 34

3.3 Verification of Proposed Equations _ . . . . . . . . . . . . 39

$\begin{array}{lll}\text { Chapter } 4 & \text { Terminal Point Guidance } & 56\end{array}$

4.1 Introduction . . . . . . . . . . . . . . . . . 56

4.2 Back-Propagation of Proposed Equations . . . . . . . . . . 57

4.2.1 Closed-Loop Implementation . . . . . . . . . . . . . . . 58

4.2 .2 Control Method Selection _ . . . . . . . . . . . . . 60

4.3 Back-Propagation Simulation Results . . . . . . . . . . . . . 61

$\begin{array}{lll}\text { Chapter } 5 & \text { Continuous Guidance and Control Applications } & 67\end{array}$

5.1 Introduction . . . . . . . . . . . . . . . . . . . . . 67

5.2 Closed-Loop G\&C Simulation . . . . . . . . . . . . . . . . 68

5.3 G\&C with Clohessy-Wiltshire Equations . . . . . . . . . . . 69

5.4 Proposed Analytical Solution Guidance System _ . . . . . . . . 73

5.5 Back-Propagation With Continuous Control . . . . . . . . . . 83

$\begin{array}{lll}\text { Chapter } 6 & \text { Conclusion } & 89\end{array}$

6.1 Summary of Thesis . . . . . . . . . . . . . . . . . . . . 89

6.2 Significance of Work . . . . . . . . . . . . . . . . . . . 91

6.3 Recommendations for Future Work . . . . . . . . . . . . . . . . 92 
Appendix A Step-By-Step Procedure for Proposed Relative Motion Solution 


\section{List of Tables}

$3.1 \quad$ Initial Osculating Orbital Elements . . . . . . . . . . . . . 40

4.1 Orbital Elements for Closed-Loop Guidance and Control Simulation ......................... 61

$4.2 \quad$ Comparison of required $\Delta v$ for the Two Simulation Cases . . . 62

5.1 Orbital Elements for Continuous G\&C System (Circular Case) 70

$5.2 \quad$ Orbital Elements for Continuous G\&C System (Elliptical Case) 72

5.3 Orbital Elements for Continuous G\&C System (Highly Elliptical

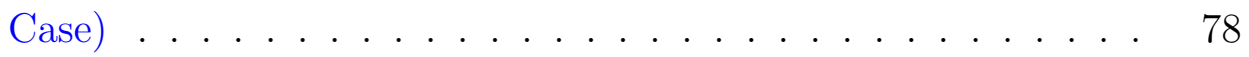

5.4 Orbital Elements for Continuous G\&C System (Back Propagation Case) . . . . . . . . . . . . . . . 85 


\section{List of Figures}

$2.1 \quad$ Diagram of reference frames. . . . . . . . . . . . 11

2.2 Diagram of semimajor axis, eccentricity vector, and true anomaly for an elliptical spacecraft orbit. . . . . . . . . . . . . . 15

2.3 Diagram showing inclination, right ascension of ascending node, and argument of perigee, the red ellipse represents the equatorial plane of the Earth, and the grey ellipse is the orbit plane.

2.4 Graphical representation of the oblateness of the Earth, which is responsible for the $J_{2}$ perturbation. . . . . . . . . . .

2.5 Block diagram for the numerical simulator used as the ground truth for spacecraft formation relative position. . . . . . . .

3.1 Figure showing the formation drifting effect caused by J2; inplane elliptical formation with $a^{\prime}=9000 \mathrm{~km}$ and $e^{\prime}=0.2$. .

3.2 Diagram showing the relative orbital elements, the green ellipse represents the orbit plane of the leader, and the grey ellipse is the orbit plane of the follower. . . . . . . . . . . . 37

3.3 Analytical solutions compared with numerical simulator. . .

3.4 Analytical solutions compared with numerical simulator for $e^{\prime}=$ 0.2 and $a^{\prime}=9,000 \mathrm{~km} \ldots \ldots \ldots \ldots \ldots \ldots$

3.5 Analytical solutions compared with numerical simulator for $e^{\prime}=$ 0.4 and $a^{\prime}=12,000 \mathrm{~km} . \ldots \ldots \ldots \ldots \ldots$

3.6 Analytical solutions compared with numerical simulator for $e^{\prime}=$ 0.8 and $a^{\prime}=35,000 \mathrm{~km} . \ldots \ldots \ldots \ldots \ldots$

3.7 Analytical solutions compared with numerical simulator for PROBA3 example. . . . . . . . . . . . . . . . . . . . . 47

3.8 Analytical solutions compared with numerical simulator for $e^{\prime}=$ 0.2 and $a^{\prime}=9,000 \mathrm{~km}$ and $i=i^{\prime}+0.05$ deg. . . . . . . 49 
3.9 Analytical solutions compared with numerical simulator for $e^{\prime}=$ 0.2 and $a^{\prime}=9,000 \mathrm{~km}$ and $i=i^{\prime}+0.1 \mathrm{deg} . \ldots . . . . .50$

3.10 Analytical solutions compared with numerical simulator for $e^{\prime}=$ 0.2 and $a^{\prime}=9,000 \mathrm{~km}$ and $i=i^{\prime}+0.15 \mathrm{deg}$. . . . . . . . 51

3.11 Comparison of error resulting from the Proposed equations of motion and the Gurfil and Kholshevnikov solution with different initial true anomaly values. . . . . . . . . . . . . .

3.12 Analytical solution compared with numerical simulator for $e^{\prime}=$

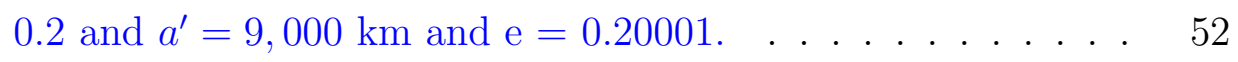

3.13 Short-periodic variation of each orbital element with time plotted with true anomaly for the $e^{\prime}=0.2$ and $a^{\prime}=9,000 \mathrm{~km}$

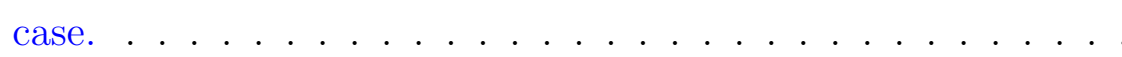

3.14 Short-periodic variation of each orbital element with time plotted with true anomaly for the $e^{\prime}=0.8$ and $a^{\prime}=35,000 \mathrm{~km}$ case. . . . . . . . . . . . . . . .

4.1 Comparison of relative position from numerical simulator and back-propagation (note that the formation is the same as defined in Fig. 3.8) . . . . . . . . . . . . . . . .

4.2 Relative position error of back-propagation compared with numerical simulator (note that the formation is the same as defined in Fig. 3.8) . . . . . . . . . . . . . . . .

4.3 Closed-loop back-propagation guidance and impulsive control performance validation for Case 1. . . . . . . . . . .

4.4 Closed-loop back-propagation guidance and impulsive control

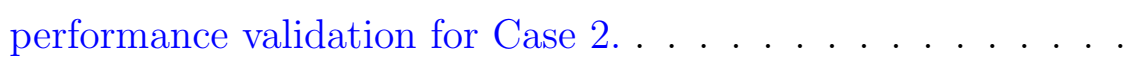

$5.1 \quad$ Schematic diagram of G\&C simulation. . . . . . . . . . 68

5.2 Validation of control effectiveness for G\&C system using HCW model in an unperturbed, circular orbit. . . . . . . . . . . 71 
5.3 Control inputs for G\&C system using HCW model in an unperturbed, circular orbit. . . . . . . . . . . . . . 72

5.4 Validation of control effectiveness for G\&C system using HCW model in a $J_{2}$-perturbed, elliptical orbit. . . . . . . . . 73

5.5 Control inputs for G\&C system using HCW model in a $J_{2^{-}}$ perturbed, elliptical orbit. . . . . . . . . . . . . . . . 74

5.6 Validation of control effectiveness for G\&C system using proposed analytical equations in a $J_{2}$-perturbed elliptical orbit. . 76

5.7 Control inputs for G\&C system using proposed analytical equations in a $J_{2}$-perturbed elliptical orbit. . . . . . . . . . 77

5.8 Comparison between HCW guidance, and proposed guidance for in-plane motion of formation case in Table 5.2 . . . . . . 78

5.9 Validation of control effectiveness for G\&C system using HCW model in a $J_{2}$-perturbed, highly elliptical orbit. . . . . . . 79

5.10 Control inputs for G\&C system using $\mathrm{HCW}$ model in a $J_{2^{-}}$ perturbed, highly elliptical orbit. . . . . . . . . . . . 79

5.11 Validation of control effectiveness for G\&C system using proposed analytical equations in a $J_{2}$-perturbed, highly elliptical orbit. . . . . . . . . . . . . . . . . .

5.12 Control inputs for G\&C system proposed analytical equations in a $J_{2}$-perturbed, highly elliptical orbit. . . . . . . . . 80

5.13 Validation of control effectiveness for G\&C system using proposed analytical equations with a $J_{2}$-invariant orbit in a $J_{2^{-}}$ perturbed, highly elliptical orbit. . . . . . . . . . . . 82

5.14 Control inputs for G\&C system using proposed analytical equations with a $J_{2}$-invariant orbit in a $J_{2}$-perturbed, highly elliptical orbit. . . . . . . . . . . . . . . . .

5.15 Comparison between HCW guidance, proposed guidance, and proposed guidance with a $J_{2}$ invariant orbit for in-plane motion of formation case in Table 5.3 . . . . . . . . . . . . . . . 
5.16 Validation of control effectiveness for G\&C system using proposed analytical equations for back-propagation guidance in Case 1. . . . . . . . . . . . . . . . . . . 86

5.17 Control inputs for G\&C system using proposed analytical equations for back-propagation guidance in Case 1. . . . . . . . . 87

5.18 Validation of control effectiveness for G\&C system using proposed analytical equations for back-propagation guidance in Case 2. . . . . . . . . . . . . . . . . . . .

5.19 Control inputs for G\&C system using proposed analytical equations for back-propagation guidance in Case 2. . . . . . . . . 88 


\section{List of Symbols}

$J_{2} \quad$ Second zonal coefficient of the Earth's gravitational potential

function, $J_{2}=1.08264 \times 10^{-3}$

$\vec{I}_{x} \quad$ Unit vector of the inertial reference frame in $\mathrm{x}$ direction

$\vec{P}_{x} \quad$ Unit vector of the perifocal reference frame in $\mathrm{x}$ direction

$\vec{L}_{x} \quad$ Unit vector of the LVLH reference frame in $\mathrm{x}$ direction

F $\quad$ Force, $\mathrm{N}$

$G \quad$ Universal gravitational constant, $6.6732 \times 10^{-11} \mathrm{Nm}^{2} / \mathrm{kg}^{2}$

m Mass, $\mathrm{kg}$

r Position, $\mathrm{km}$

$\mu \quad$ Gravitational constant, typically for Earth $\left(\mu_{\text {Earth }}=3.986 \times 10^{5} \mathrm{~km}^{3} / \mathrm{s}^{2}\right.$

a Semimajor axis, $\mathrm{km}$

$e \quad$ Eccentricity, unitless

$i \quad$ Inclination, rad

$\Omega \quad$ Right ascension of ascending node, rad

$\omega \quad$ Argument of perigee, rad

$\nu \quad$ True anomaly, rad

$\epsilon \quad$ Orbital energy

$h \quad$ Orbital angular momentum, $\mathrm{km}^{2} / \mathrm{s}$

$N \quad$ Orbit normal

$u \quad$ Argument of latitude, rad

$p \quad$ Semilatus rectum, $\mathrm{km}$

E Eccentric anomaly, rad

$M \quad$ Mean anomaly, rad

$n \quad$ Mean orbital motion, $\mathrm{rad} / \mathrm{s}$

$t \quad$ Time, s

$t_{p} \quad$ Time since perigee passage, $\mathrm{s}$ 


$\begin{array}{ll}C_{P I} & \text { Rotation matrix from inertial to perifocal frame } \\ \boldsymbol{C}_{L P} & \text { Rotation matrix from perifocal to LVLH frame } \\ \boldsymbol{C}_{L I} & \text { Rotation matrix from inertial to LVLH frame } \\ f & \text { Perturbing acceleration, } m / s^{2} \\ a & \text { Acceleration, } m / s^{2} \\ K_{p} & \text { Proportional controller constant, } 1 / \mathrm{s}^{2} \\ K_{d} & \text { Derivative controller constant, } 1 / \mathrm{s} \\ e & \text { Position error, } \mathrm{km} \\ \rho & \text { relative position, } \mathrm{km} \\ \Delta v & \text { Acceleration expressed as change in velocity } \\ \dot{x} & \text { Time derivative of } \mathrm{x} \\ \ddot{x} & \text { Second time derivative of } \mathrm{x} \\ \vec{x} & \text { Physical vector of } \mathrm{x} \\ \boldsymbol{x} & \text { Components of physical vector of } \mathrm{x} \\ \bar{x} & \text { Mean value of } \mathrm{x} \\ \delta x & \text { Difference in orbital element } \mathrm{x}\end{array}$

\section{Subscripts}

$\begin{array}{ll}I & \text { Inertial reference frame } \\ P & \text { Perifocal reference frame } \\ L & \text { LVLH reference frame } \\ x & \text { x direction } \\ y & \text { y direction } \\ z & \text { z direction } \\ 0 & \text { Initial value } \\ s p & \text { Short-periodic variation }\end{array}$




\section{Superscripts}

$\begin{array}{ll}\prime & \text { Leader Spacecraft (lack of this superscript implies follower spacecraft) } \\ * & \text { Relative orbital element } \\ \times & \text { Skew symmetric operation } \\ -1 & \text { Inverse }\end{array}$




\section{List of Acronyms}

$\begin{array}{ll}\text { ESA } & \text { European Space Agency } \\ \text { G\&C } & \text { Guidance and control } \\ \text { LVLH } & \text { Local-vertical-local-horizontal reference frame } \\ \text { HCW } & \text { Hill-Clohessy-Wiltshire, referencing the Hill-Clohessy-Wiltshire } \\ & \text { equations for spacecraft relative motion } \\ \text { LTI } & \text { Linear-time-invariant } \\ \text { LPV } & \text { Linear-parameter-varying } \\ \text { LTV } & \text { Linear-time-varying } \\ \text { PD } & \text { Proportional-derivative, in reference to a controller } \\ \text { ECI } & \text { Earth centered inertial reference frame } \\ \text { RAAN } & \text { Right ascension of ascending node }\end{array}$




\section{Chapter 1}

\section{Introduction}

\subsection{Motivation}

Multiple spacecraft formation flying is rapidly becoming a key space technology, which increases the efficiency, cost effectiveness and flexibility of an operational mission. Examples of spacecraft formation flying missions include GRACE,[1] TanDEM-X,[2] and PRISMA.[3] Despite the success of these past missions, future formation flying missions such as the ESA PROBA-3 mission will have to satisfy increasingly challenging functional and performance requirements in their guidance, navigation, and control systems. In particular, guidance and control (G\&C) algorithms and their implementation must comply with limited on-board processing resources and propellant. The guidance system is responsible for calculating the desired relative motion trajectory to be tracked by the control system during reconfiguration maneuvers between the initial (current) and the desired (future) formation defined by the user. It is also responsible for providing a reference signal for the desired motion for formation keeping maneuvers. The efficiency of formation flying maneuvers in terms of propellant usage is directly related to the accuracy of the guidance system, while the efficiency in terms of on-board computational resources is directly related to the simplicity of the equations used for guidance. Balancing accuracy and simplicity in spacecraft guidance algorithms is key to the future development of spacecraft formation flying missions, and is the motivation behind this work.

\subsection{Problem Statement}

The trade-off between accuracy and simplicity is found throughout the field of spacecraft dynamics. Methods for propagating highly-accurate spacecraft motion in time are well-known and easy to implement, but typically rely on numerical integration 
to solve the differential equations of motion. The amount of computing power on board a spacecraft is typically severely limited, making guidance equations relying on numerical integration undesirable. This is especially true for spacecraft formation flying, where the motion of one spacecraft relative to another is necessary for guidance purposes, and therefore the motion of two spacecraft must be considered.

Simple analytical solutions for relative motion are also found throughout the field. Though these solutions are computationally simple, they are often not accurate enough for efficient guidance. Specifically, these analytical solutions typical neglect orbit perturbations, make assumptions about the eccentricity of the orbit, or some combination of these and other simplifying assumptions. The resulting sets of equations of relative motion are useful for Keplerian orbits ${ }^{1}$, but are not particularly useful in real-world scenarios where greater accuracy is required.

In this context, this thesis is concerned with deriving a set of equations of relative motion for two spacecraft in formation which is analytical, yet still accounts for noncircular orbits and the largest orbital perturbation $\left(J_{2}\right)$, resulting in a computationally efficient, yet accurate relative motion solution for use in guidance applications.

In a recent survey of the guidance, navigation and control of spacecraft formations, Di Mauro et al. state that the relative position of spacecraft in formation should be controllable to an accuracy of two orders of magnitude less than the spacecraft separation distance.[4] This general desired formation accuracy given is validated by looking at some past formation flying missions and the relative position accuracy that they were able to achieve. For example, the TanDEM-X mission's required control accuracy was 20 meters in the radial and crosstrack directions and 200 meters in the alongtrack direction, with spacecraft separation ranging from 150 meters up to a few kilometers $[2,4]$, and the PRISMA mission required position control of 25 meter accuracy with separation distances ranging from 100 to 2000 meters. [3] The CanX-4 and CanX-5 spacecraft, two small satellites used to demonstrate precision spacecraft formation flying, were able to achieve control accuracy to within a meter, with relative separation distances of approximately 50 to 1000 meters. [4, 5] It should be noted that each of these spacecraft formations involves relatively low eccentricity orbits, and that

\footnotetext{
${ }^{1}$ A Keplerian orbit assumes a spacecraft of negligible mass orbiting a body of point mass (or equivalent to a point mass) with no external perturbations
} 
increasing the eccectricity of the orbits in the formation would tend to derease the accuracy of the relative position control.

Although accuracy requirements may vary greatly depending on the mission profile, these generalized accuracy requirements are a good baseline with which to compare the accuracy of a newly proposed set of relative motion equations. Thus, a suitable goal for the proposed analytical solution for spacecraft relative motion is that it maintain accuracy of at least two orders of magnitude less than the spacecraft separation distance even in high eccentricity, $J_{2}$-perturbed orbits. The computational efficiency of the proposed solution is a goal secondary to the accuracy, and therefore the focus of this thesis is not to compare the computational efficiency with any baseline. Instead, the computational efficiency is implicit in the requirement that the proposed relative motion solution is analytical.

\subsection{Previous Work}

Although there are analytical solutions for the exact non-linear differential equations of relative motion on the local-vertical-local-horizontal (LVLH) reference frame, these solutions have their limitations as a result of imposing certain restrictions in their deviations. The Hill-Clohessy-Wiltshire (HCW) model [6] is a linearized example where the derived time-explicit closed-form analytical solution is only valid for circular Keplerian orbits. Work by Sabol and McLaughlin [7] used this model to demonstrate that the in-plane and out-of-plane non-drifting relative motion about a circular Keplerian orbit always follows an ellipse centered on the reference orbit. To overcome the inherent limitations of the HCW model, some formulations also take into account orbit perturbations, such as the $J_{2}$ perturbation. The $J_{2}$ perturbation is particularly important in formation flying since its secular effects on an orbit, i.e., the rotation of the line of apsides and precession of the line of nodes, cause secular drift between two $J_{2}$-perturbed spacecraft. The introduction of $J_{2}$ in a linearized set of equations, similar to the HCW equations, has been proposed by Schweighart and Sedwick.[8] Specifically, the authors developed a set of linear, constant-coefficient, second-order

differential equations of motion by considering the orbit-averaged impact of $J_{2}$ on a circular reference orbit. An analytical solution to these differential equations is also 
presented.

However, assuming a circular reference orbit yields considerable errors when the eccentricity of the reference orbit increases. In fact, it has been shown that the errors introduced in the HCW equations by considering an elliptical reference orbit dominate the errors due to ignoring $J_{2} \cdot[9]$ For this reason, several formulations have been proposed to model the relative motion about unperturbed elliptical orbits.[10, 11, 12] In particular, the linear-time-invariant (LTI) HCW equations have been extended to arbitrary eccentricity by Inalhan et al.[9] by formulating the dynamics as a linearparameter-varying (LPV) system. Such a dynamics model is especially well suited for controller design purposes, by making use of LPV control techniques, such as model predictive control.[13] Making use of the fact that the orbit angular rate and the radius are functions of the true anomaly, these equations can also be expressed using true anomaly derivatives instead of time derivatives.[14] The resulting differential equations are also time-varying, but are not parameter-varying since the true anomaly has been used to formulate the derivatives. Solutions to these linear-timevarying (LTV) equations are available in the literature in various forms using different reference frames and variables. The first derivation with singularities in the closed-form solution was provided by Lawden.[15] Then, Carter [16] provided a set of solutions without singularities. These homogenous solutions are extremely useful for well-behaved numerical and analytical analysis on the shape, structure, and optimization of passive apertures in eccentric reference orbits. Interestingly, the same solutions can be obtained via incremental changes in orbital elements, as demonstrated by Marec.[17] Lane and Axelrad [18] developed a time-explicit closed-form solution and studied the relative motion for bounded elliptical orbits. Melton [19] also proposed an alternative solution for small eccentricity reference orbits. Recent work from Gufanti et al.[20] approaches the problem of analytical propagation of spacecraft relative motion in perturbed, eccentric orbits using a relative orbital element state representation augmented with force model parameters. The resulting formulations capture the secular and long-periodic effects of the perturbations, but average out the short-periodic effects.

Recently, a closed-form analytical solution that is valid for Keplerian eccentric 
orbits was developed by Gurfil and Kholshevnikov.[21, 22] This simple analytical solution explicitly parametrizes the relative motion using classical orbital elements as constants of the unperturbed Keplerian orbit, instead of Cartesian initial conditions. This concept, originally suggested by Hill, [23] has been widely used in the analysis of relative spacecraft dynamics.[24, 25]

\subsection{Thesis Objectives}

The objective of this thesis is to develop a novel analytical solution for the relative motion of spacecraft formations on $J_{2}$-perturbed, arbitrary eccentricity orbits, validate the proposed equations, and to apply them in examples of their applications.

This thesis first expands the work of Gurfil and Kholshevnikov [21, 22] by using $J_{2^{-}}$ perturbed orbital elements in their solution. A key advantage of this approach is that it facilitates the inclusion of the orbital perturbations in the relative motion equations. This can namely be achieved by using $J_{2}$-perturbed osculating orbital elements in their solutions instead of using constant orbital elements, where perturbed osculating orbital elements are calculated by adding the mean orbital elements to the $J_{2}$-induced short-periodic variations. [26, 27] The accuracy of the proposed analytical solution is then validated through comparison with a numerical simulator. Finally, this thesis investigates a number of practical applications of the proposed equations as guidance for reconfiguration and formation keeping maneuvers. The resulting guidance laws are then validated in a closed-loop G\&C simulations, both with impulsive and continuous controllers. These scenarios demonstrate the efficiency of the proposed guidance in terms of control acceleration, which is directly related to propellant usage.

\subsection{Contributions}

The contributions of this work to the field of spacecraft formation flying guidance and control are:

- The develoment of a novel set of non-linear analytical equations of motion describing the relative motion of spacecraft in formation in the LVLH reference 
frame on $J_{2}$-perturbed, eccentric orbits

- The validation of this set of equations against results obtained from a numerical simulator

- The development of a simple, back-propagation guidance methodology which makes use of the proposed equations of motion

- The demonstration of the practical application of this guidance algorithm in closed-loop control scenarios

\subsection{Organization}

This thesis is organized as follows:

A variety of concepts important to the thesis are first outlined in Chapter 2: Basic Concepts, culminating in the definition of the numerical simulator used as a reference signal for true spacecraft motion. This includes defining important reference frames which will be used throughout the thesis, defining the equations of motion for spacecraft, discussing orbital elements and perturbations, and defining an existing guidance system to compare with the novel guidance system which will be developed.

The derivation of the novel, non-linear analytical equations of motion for spacecraft formations in $J_{2}$-perturbed, eccentric orbits is then defined in Chapter 3: Proposed Equations of Motion. This Chapter forms the bulk of this thesis, outlining the procedure undergone to derive the proposed equations. The equations are also compared with the numerical simulator in this section to verify their accuracy.

Chapter 4: Terminal Point Guidance details one practical application of the proposed equations of motion in a closed-loop guidance and control scenario. The concept of a back-propagation guidance methodology is first detailed, and the equations of motion are proven to be capable of accurately propagating the motion of a spacecraft 
formation both backwards and forwards in time. A scenario and corresponding simulation is then set up to demonstrate the effectiveness of the newly-developed guidance system.

More practical applications of the proposed equations of motion are then discussed in Chapter 5: Continuous Guidance and Control Applications. This section includes the comparison of several G\&C simulations involving closed-loop control with a timecontinuous PD controller using the proposed equations of motion as the guidance system of a spacecraft.

This thesis ends with Chapter 6: Conclusion, which summarizes the contributions of this work and proposes future work which could be undertaken to extend this work. 


\section{Chapter 2}

\section{Basic Concepts}

The goal of this chapter is to introduce the reader to concepts and pertinent background information related to spacecraft formation flying, and orbital mechanics in general. A brief overview is given of a variety of topics, including important reference frames, spacecraft motion on Keplerian orbits, orbital elements, orbit perturbations, and the numerical simulation of spacecraft dynamics.

\section{$2.1 \quad$ Introduction}

This chapter of the thesis provides detailed background information for numerous concepts in spacecraft dynamics and spacecraft formation flying. These basic concepts will be used throughout this thesis.

First, Sec. 2.2: Reference Frames, presents a discussion of important reference frames which will be used throughout this thesis to describe spacecraft position and velocity.

This is followed by an overview of spacecraft motion in Keplerian (unperturbed) orbits, using the differential two-body equations of motion, in Sec. 2.3: Spacecraft Motion on Keplerian Orbits. The two-body equations of motion are a necessary step towards more sophisticated dynamics models and form the basis of much of the work of this thesis. Additionally, because the equations are an exact solution to the spacecraft motion in the unperturbed case, these equations of motion are a suitable baseline case against which to compare the accuracy of other results.

Section 2.4: Orbital Elements presents six classical orbital elements and their relation to the position and velocity of a spacecraft. These orbital elements are the most convenient method of initializing the orbits of spacecraft, even in formation. They are also central to the proposed analytical method of calculating spacecraft motion to be derived in the next chapter of the thesis. 
The reference frames used in this thesis and the concept of orbital elements can be combined to define rotations matrices. The important rotations are discussed in Sec. 2.5 Rotation Matrices.

The goal of this thesis is to find an analytical solution to spacecraft relative motion which accounts for the $J_{2}$ perturbation. Perturbations will be discussed briefly in Sec. 2.6: Perturbations, with special emphasis on $J_{2}$. Additionally, the equations used for calculating the perturbing acceleration of the $J_{2}$ perturbation will be presented.

In Sec. 2.7: Numerical Simulator, the equations defined in previous sections will be used to set up a simulator for numerically solving the two-body equations of motion, with the $J_{2}$ perturbing acceleration for spacecraft motion. This section will also discuss the implementation of rotations between reference frames to ultimately obtain the relative motion of a follower spacecraft in reference to a leader. This numerical simulator is used throughout the thesis as a baseline with which to compare new analytical solutions for the spacecraft formation motion.

Section 2.8: Hill-Clohessy-Wiltshire Model for Formation Flying presents one existing set of equations of spacecraft relative motion. This model for formation flying guidance will be used later in the thesis for comparison with the newly-developed equations of relative motion.

\subsection{Reference Frames}

For completeness, the reference frames that will be subsequently used in the derivations of the equations of motion are first introduced.

The first is the Earth-centered inertial (ECI) reference frame, denoted by $\mathcal{F}_{I}$ and

defined by its orthonormal unit vectors $\left\{\vec{I}_{x}, \vec{I}_{y}, \vec{I}_{z}\right\}$. This reference frame has its origin at the center of the Earth, its $\vec{I}_{z}$ is along the Earth's polar axis of rotation, and its $\vec{I}_{x}$ and $\vec{I}_{y}$ unit vectors in the Earth's equatorial plane. Specifically, the unit vector $\vec{I}_{x}$ of the ECI frame is along the vernal equinox line (the intersection of the Earth's equatorial plane and the ecliptic plane moving from south to north) in the direction of the vernal equinox (sometimes called the first point of Aries), and $\vec{I}_{y}$ completes the right handed triad $\left(\vec{I}_{y}=\vec{I}_{Z} \times \vec{I}_{x}\right)$.

The perifocal frame, denoted by $\mathcal{F}_{P}$ and defined by its orthonormal unit vectors 
$\left\{\vec{P}_{x}, \vec{P}_{y}, \vec{P}_{z}\right\}$, is also centered at the center of the Earth, but with its $\vec{P}_{z}$ aligned with a spacecraft's orbit normal vector, its $\vec{P}_{x}$ pointing in the direction of the perigee, and $\vec{P}_{y}=\vec{P}_{z} \times \vec{P}_{x}$. This frame is specific to the orbit of a given spacecraft. As a result, two seperate perifocal frames will be used in this paper, i.e., one for each spacecraft. These frames will be denoted $\mathcal{F}_{P}$ and $\mathcal{F}_{P^{\prime}}$ for the perifocal frame of the follower and the leader spacecraft, respectively. The corresponding unit vectors will be labelled $\left\{\vec{P}_{x}, \vec{P}_{y}, \vec{P}_{z}\right\}$ and $\left\{\vec{P}_{x}^{\prime}, \vec{P}_{y}^{\prime}, \vec{P}_{z}^{\prime}\right\}$ for the follower and leader spacecraft, respectively.

The last reference frame relevant to this work is the local-vertical-local-horizontal (LVLH) reference frame denoted by $\mathcal{F}_{L}$ and defined by its orthonormal unit vectors $\left\{\vec{L}_{x}, \vec{L}_{y}, \vec{L}_{z}\right\}$. This reference frame has its origin at the leader spacecraft, with its $\vec{L}_{x}$ unit vector aligned with the spacecraft position vector (defined as the vector from the center of the Earth to the spacecraft's current position and labelled $\vec{r}$ ) and its $\vec{L}_{z}$ unit vector aligned with the orbit normal. The $\vec{I}_{y}$ unit vector completes the right handed triad. Note that in the case of a perfectly circular orbit, $\vec{I}_{y}$ is in the direction of the velocity vector, and is sometimes referred to as the along-track direction. Similarly, $\vec{L}_{x}$ may be referred to as the radial direction, and $\vec{L}_{z}$ is sometimes called the out-of-plane direction. The solution to the equations of motion developed in this thesis provides an analytical expression of the position of the follower spacecraft with respect to the leader in $\mathcal{F}_{L}$.

A diagram showing these three important reference frames is shown in Fig. 2.1.

\subsection{Spacecraft Motion on Keplerian Orbits}

The motion of spacecraft in orbit around some central body is described using Newton's Law of Gravitation. This law states that the force of gravity which acts on one object as a result of another is related to the mass of those objects and the squared distance between them, and the direction of the force will be along the line connecting the two objects. Mathematically, Newton's law of gravitation is expressed in the following equation

$$
\vec{F}_{21}=-\frac{G m_{1} m_{2}}{\left|\vec{r}_{21}\right|^{3}} \vec{r}_{21}
$$




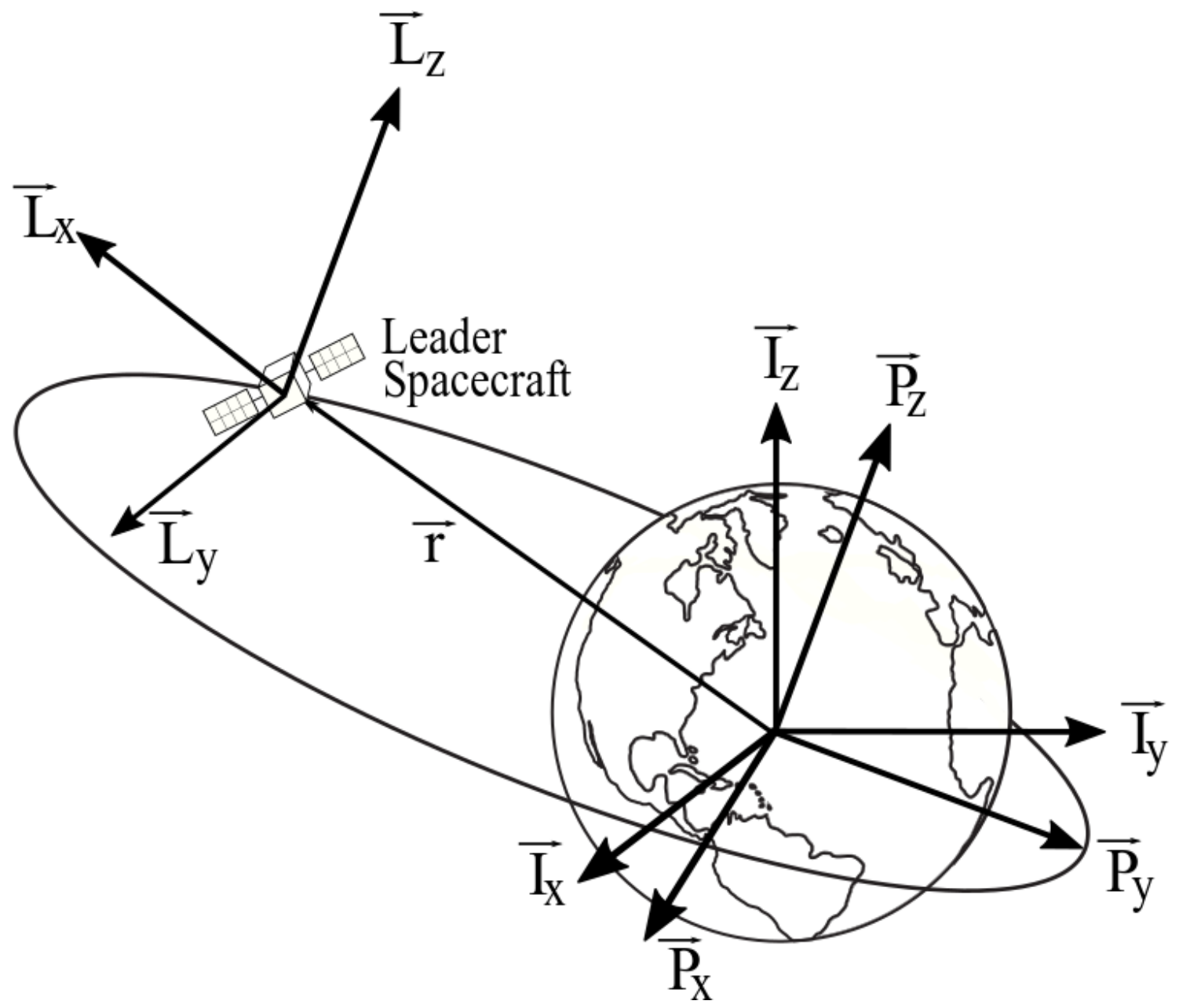

Figure 2.1: Diagram of reference frames. 
where $\vec{r}_{21}$ is the position vector between the two objects, $m_{1}$ and $m_{2}$ are their respective masses, and the universal gravitational constant, $G$ is equal to $6.6732 \times$ $10^{-11} \mathrm{Nm}^{2} / \mathrm{kg}^{2}$. Using Newton's second law, the gravitation force found using Eq. (2.1) can be related to the acceleration of the masses. Specifically, the following two equations can be written as

$$
\begin{gathered}
m_{1} \ddot{\overrightarrow{R_{1}}}=\vec{F}_{12}=\frac{G m_{1} m_{2}}{\left|\vec{r}_{21}\right|^{3}} \vec{r}_{21} \\
m_{2} \ddot{\overrightarrow{R_{2}}}=\vec{F}_{21}=-\frac{G m_{1} m_{2}}{\left|\vec{r}_{21}\right|^{3}} \vec{r}_{21}
\end{gathered}
$$

where the absolute acceleration of each body can be simplified as follows

$$
\begin{gathered}
\ddot{\overrightarrow{R_{1}}}=\frac{G m_{2}}{\left|\vec{r}_{21}\right|^{3}} \vec{r}_{21} \\
\ddot{\overrightarrow{R_{2}}}=-\frac{G m_{1}}{\left|\vec{r}_{21}\right|^{3}} \vec{r}_{21}
\end{gathered}
$$

where the relative acceleration between the two bodies, in this case a large central body (typically a planet) and a spacecraft, is of most interest. Taking the spacecraft as object 2 , the relative acceleration of the spacecraft with respect to the central body can be expressed as the following (in the ECI reference frame)

$$
\ddot{\vec{r}}=\ddot{\overrightarrow{R_{2}}}-\ddot{\overrightarrow{R_{1}}}=-\frac{\mu}{r^{3}} \vec{r}
$$

where $\mu$ is called the gravitational constant of the two-body system, and is defined as follows

$$
\mu=G\left(m_{1}+m_{2}\right) \approx G m_{1}
$$

The approximation is made based on the fact that spacecraft masses are many orders of magnitude lower than the mass of the central body which they orbit. For example, the mass of a large Earth-orbiting spacecraft may be somewhere in the order of several thousand kilograms, while the mass of the the Earth itself is $5.972 \times 10^{24}$ kilograms. The gravitational constant for any Earth orbit is evaluated to be $3.986 \times 10^{5} \mathrm{~km}^{3} / \mathrm{s}^{2}$. 
Equation (2.6) defines the orbit of a spacecraft in two-body, unperturbed motion around a central body, and forms the basis of the equations of motions used in this thesis. This equation can be integrated twice at any given time in the orbit of the spacecraft to find the position vector, $\vec{r}(t)$, and velocity vector, $\dot{\vec{r}}(t)$, defining the motion of the spacecraft. It should be noted that to perform this integration an initial position and velocity vector are required, or the three initial components of the position vector and the three initial components of the velocity vector in the ECI frame for an Earth-orbiting spacecraft.

\subsection{Orbital Elements}

As stated at the end of the previous section, six total position and velocity components are required to integrate the equation for orbital motion and obtain the position and velocity of a spacecraft. In other words, given some initial position and velocity vectors, an orbit is uniquely defined, with each combination of initial conditions resulting in a different size, shape and orientation of orbit. However, the initial position and velocity vectors do not provide an intuitive set of initial conditions if particular orbit shapes, sizes and orientations are sought. Instead of using initial position and velocity, a set of six scalar values which define the size, shape and orientation of an orbit called classical orbital elements are often used as initial conditions. The orbital elements are represented graphically in Figs. 2.2 and 2.3 and may be defined as follows, in the context of Earth-orbiting spacecraft:

- $a$ - Semimajor axis $(\mathrm{km})$ : defined as the distance between the centre point of an elliptical orbit and the perigee, point (closest approach to the Earth). The semimajor axis defines the size of the orbit.

- $e$ - Eccentricity (unitless): a measure of how elliptical the orbit is, with 0 indicating a circular orbit, and anything greater or equal to 1 indicating an orbit which is not closed. Note that $e=|\vec{e}|$. The eccentricity vector, $\vec{e}$ will be defined in Sec. 2.4.1.

- $i$ - Inclination (rad): the angle between the orbital plane and the equatorial plane of the Earth. 
- $\Omega$ - Right ascension of ascending node (RAAN) (rad): The angle between the $\vec{I}_{x}$ unit vector in the ECI frame and the ascending node, defined as the point on the line of nodes (the line of intersection between the equatorial and orbit planes) where the orbit crosses from the southern to northern hemisphere.

- $\omega$ - Argument of perigee (rad): Defined as the angle between the ascending node and the perigee of the orbit. The inclination, right ascension of ascending node, and argument of perigee describe the orientation of the orbit.

- $\nu$ - True anomaly (rad): Describes the current position of the spacecraft in its orbit based on time. The true anomaly is the angle between the position vector of the spacecraft at perigee and the current position vector. The true anomaly is one of many possible measures describing the current position of the spacecraft in the orbit. It can be directly related to time through the time of perigee passage. It is also related to eccentric anomaly, and mean anomaly, with mean anomaly being especially important in this thesis. Mean anomaly is the angle between the position vector at perigee, and the current position vector of the spacecraft if it is assumed to move with a constant angular rate with time, called the mean orbital motion $(n)$.

Orbital elements will play an important role in the proposed equations of relative motion to be defined in the next chapter of this thesis. As a result of this, it is often necessary to be able to change between orbital elements and initial position and velocity vectors, such that the position and velocity vectors can be used to initialize numerical simulations which integrate the equation of motion given in Eq. (2.6).

\subsubsection{Conversion from Inertial Position and Velocity to Orbital Elements}

The orbital elements of a given orbit can be determined from the inertial position and velocity of a spacecraft through the following process.

First, the semimajor axis can be calculated based on the orbital energy and the gravitational parameter, using the following equation

$$
a=-\frac{\mu}{2 \varepsilon}
$$




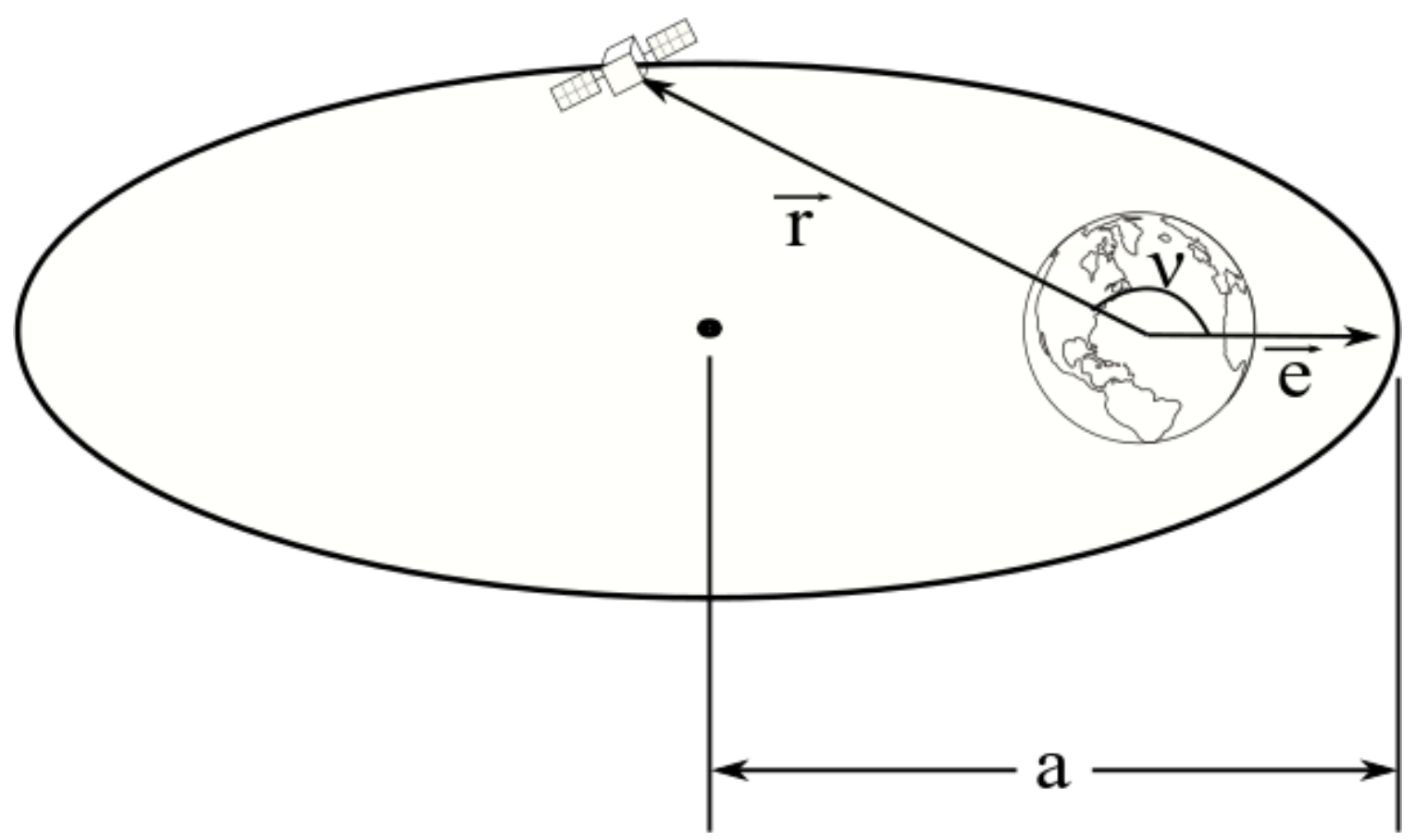

Figure 2.2: Diagram of semimajor axis, eccentricity vector, and true anomaly for an elliptical spacecraft orbit.

where $\varepsilon$ is the specific orbital energy for the orbit, which can be calculated as follows

$$
\varepsilon=\frac{v^{2}}{2}-\frac{\mu}{r}
$$

where $r$ and $v$ are the position and speed of the spacecraft respectively, that is, $r=|\vec{r}|$ and $v=|\vec{v}|$.

The eccentricity, $e$, is the magnitude of the eccentricity vector $(e=|\vec{e}|)$, which is calculated from the position and velocity vectors as follows

$$
\vec{e}=\frac{\vec{v} \times \vec{h}}{\mu}-\frac{\vec{r}}{r}
$$

with the specific orbital angular momentum, $\vec{h}$, defined by

$$
\vec{h}=\vec{r} \times \vec{v}
$$

The inclination angle is defined as the angle between the orbital angular momentum vector and $\vec{I}_{z}$, the direction of the $\mathrm{z}$ unit vector in the inertial frame. Using the 


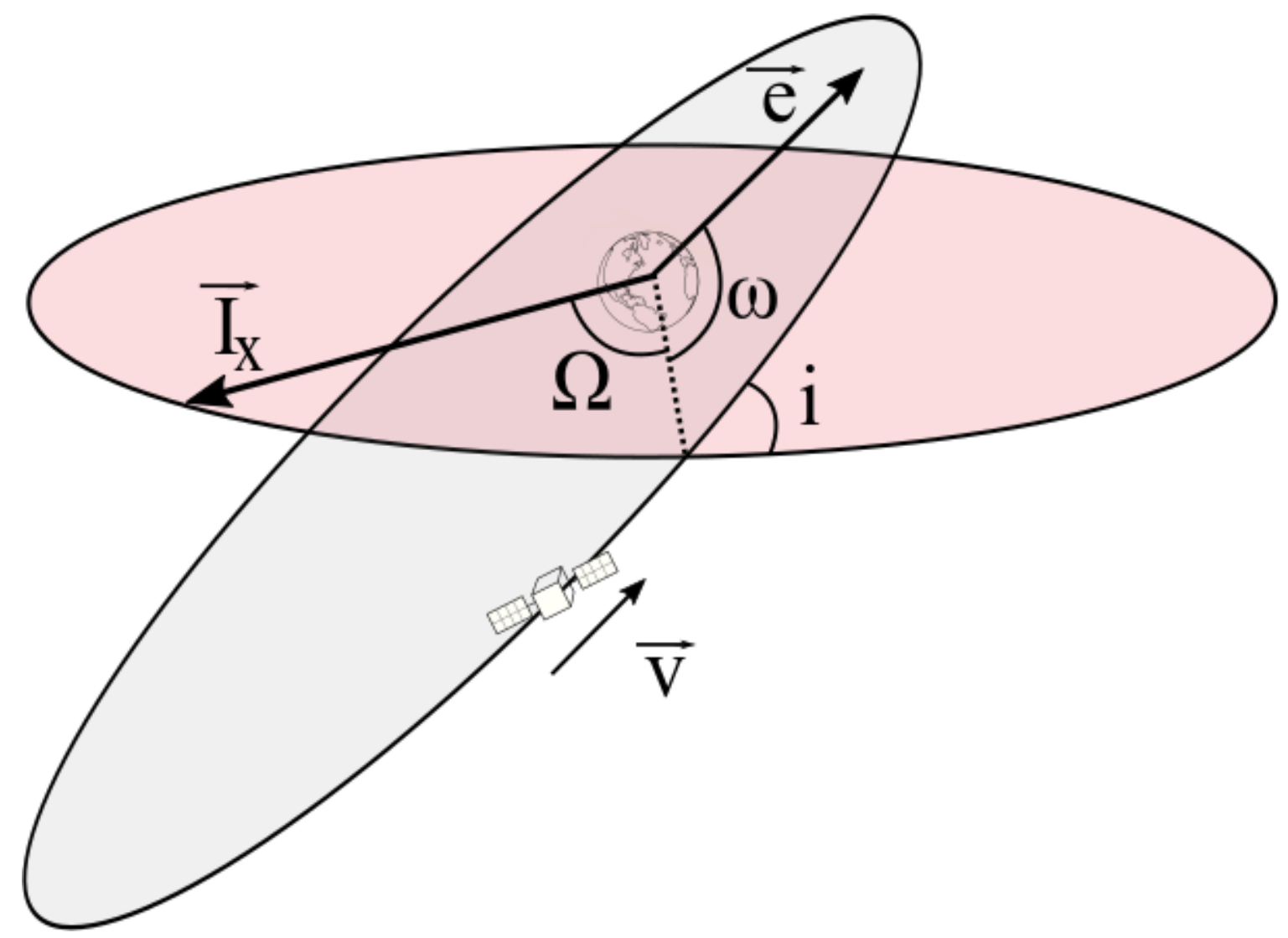

Figure 2.3: Diagram showing inclination, right ascension of ascending node, and argument of perigee, the red ellipse represents the equatorial plane of the Earth, and the grey ellipse is the orbit plane.

dot product, the angle $i$ can be expressed as follows

$$
i=\cos ^{-1}\left\{\frac{\vec{h} \cdot \vec{I}_{z}}{h}\right\}
$$

where $h$ is the magnitude of the orbital angular momentum vector.

The right ascension of ascending node is the the angle between the ascending node and $\vec{I}_{x}$, the unit vector of the ECI frame in the x direction. The ascending node is defined by the cross product of the $\vec{I}_{z}$ vector and the orbital angular momentum vector, as follows

$$
\vec{N}=\vec{I}_{z} \times \vec{h}
$$

The right ascension of the ascending node is then defined using the dot product, 
similarly to the inclination, as

$$
\Omega=\cos ^{-1}\left\{\frac{\vec{N} \cdot \vec{I}_{x}}{N}\right\}
$$

where $N=|\vec{N}|$. The correct quadrant for the right ascension of ascending node can be determined as follows

$$
\Omega= \begin{cases}0 \leq \Omega \leq 2 \pi \mathrm{rad}, & \text { if } \vec{N} \cdot \vec{I}_{y} \geq 0 \\ 2 \pi<\Omega<4 \pi \mathrm{rad}, & \text { if } \vec{N} \cdot \vec{I}_{y}<0\end{cases}
$$

The argument of perigee is defined as the angle between the direction of the ascending node and the direction of perigee, which is aligned with the eccentricity vector. Once again the dot product is used to find the following equation

$$
\omega=\cos ^{-1}\left\{\frac{\vec{N} \cdot \vec{e}}{N e}\right\}
$$

with the correct quadrant for the resulting angle being defined as follows.

$$
\omega= \begin{cases}0 \leq \omega \leq 2 \pi \mathrm{rad}, & \text { if } \vec{e} \cdot \vec{I}_{z} \geq 0 \\ 2 \pi<\omega<4 \pi \mathrm{rad}, & \text { if } \vec{e} \cdot \vec{I}_{z}<0\end{cases}
$$

Finally, to find the true anomaly, the argument of latitude, $u$, is defined as the angle between the direction of the ascending node and the position vector. The dot product is used to solve for this angle as follows

$$
u=\cos ^{-1}\left\{\frac{\vec{N} \cdot \vec{r}}{N r}\right\}
$$

with the correct quadrant for the angle being defined as follows.

$$
u= \begin{cases}0 \leq u \leq 2 \pi \mathrm{rad}, & \text { if } \vec{r} \cdot \vec{I}_{z} \geq 0 \\ 2 \pi<u<4 \pi \mathrm{rad}, & \text { if } \vec{r} \cdot \vec{I}_{z}<0\end{cases}
$$

The true anomaly is then defined as the difference between the argument of latitude and the argument of perigee, as follows 


$$
\nu=u-\omega
$$

\subsubsection{Conversion from Orbital Elements to Inertial Position and Velocity}

The inverse of this process, calculating inertial position and velocity vectors from orbital elements, can also be defined here. Typically, this is done by first finding the magnitude of the position vector as follows

$$
r=\frac{p}{1+e \cos (\nu)}
$$

where $p=a\left(1-e^{2}\right)$ is the semilatus rectum. Then the components of the position and velocity vectors can be defined in the perifocal reference frame as follows

$$
\begin{gathered}
\boldsymbol{r}_{P}=\left[\begin{array}{c}
r \cos (\nu) \\
r \sin (\nu) \\
0
\end{array}\right] \\
\boldsymbol{v}_{P}=\left[\begin{array}{c}
-\sqrt{\frac{\mu}{p}} \sin (\nu) \\
\sqrt{\frac{\mu}{p}}(e+\cos (\nu)) \\
0
\end{array}\right]
\end{gathered}
$$

Finally the inverse of the rotation matrix given in Eq. (2.30) (defined in Sec. 2.5) can be used to rotate these components into the inertial frame

$$
\begin{aligned}
& \boldsymbol{r}_{I}=\boldsymbol{C}_{I P}(\omega, i, \Omega) \boldsymbol{r}_{P} \\
& \boldsymbol{v}_{I}=\boldsymbol{C}_{I P}(\omega, i, \Omega) \boldsymbol{v}_{P}
\end{aligned}
$$

\subsubsection{Anomaly Values}

As previously mentioned, there are mutiple ways to express the sixth orbital element, with each expressing the position vector of the spacecraft relative to the perigee in a slightly different way. This section aims to further define the relationships between 
those different "anomaly" values. The eccentric anomaly $E$ can be defined as the angle between perigee and a vector from the center of the elliptical orbit to the position of the spacecraft. This angle can be related to the true anomaly through the following

$$
\tan \left(\frac{\nu}{2}\right)=\sqrt{\frac{1+e}{1-e}} \tan \left(\frac{E}{2}\right)
$$

It can easily be seen, when looking at Eq. (2.26), that true anomaly is equal to eccentric anomaly when eccentricty is zero.

The mean anomaly represents the angle between perigee and the spacecraft position vector if the spacecraft is assumed to move at a constant angular rate. It can be defined as a function of eccentric anomaly as follows

$$
M=E-e \sin (E)
$$

Mean anomaly can also be defined based on the time since perigee passage through the following equation

$$
M=n\left(t-t_{p}\right)
$$

where mean orbital motion is defined as follows

$$
n=\sqrt{\frac{\mu}{a^{3}}}
$$

and where $t$ is the current time while $t_{p}$ is the time of perigee passage. In other words, $t-t_{p}$ is the time since perigee passage.

These three anomaly values and the time since perigee passage are closely related, and using each of these values as the sixth orbital element has its own potential benefits and drawbacks. In this thesis, true anomaly will most often be used as an intuitive measure of the current angular position of a spacecraft. However, mean anomaly will be used when attempting to account for perturbation effects, with the eccentric anomaly being used to convert between mean and true anomaly. 


\subsection{Rotation Matrices}

It is necessary to be able to describe vectors, like the spacecraft position or velocity, in terms of the components in the reference frames described in Sec. 2.2. The reference frames can be related to one another using sets of rotations about the principal axes through angles which correspond to the orbital elements described in the previous section. The ECI frame can be related to the perifocal frame with a 3-1-3 rotation (three subsequent rotations through the $\mathrm{z}, \mathrm{x}$ and $\mathrm{z}$ axis) through three of the orbital elements. The resulting rotation matrix is given by

$$
\boldsymbol{C}_{P I}(\omega, i, \Omega)=\left[\begin{array}{ccc}
c_{\Omega} c_{\omega}-s_{\Omega} s_{\omega} c_{i} & s_{\Omega} c_{\omega}+c_{\Omega} s_{\omega} c_{i} & s_{\omega} s_{i} \\
-c_{\Omega} s_{\omega}-s_{\Omega} c_{\omega} c_{i} & -s_{\Omega} s_{\omega}-c_{\Omega} c_{\omega} c_{i} & c_{\omega} s_{i} \\
s_{\Omega} s_{i} & -c_{\Omega} s_{i} & c_{i}
\end{array}\right]
$$

Note that Equation 2.30 can be used to convert to either the leader or the follower's specific perifocal frame depending on whether the leader or follower orbital elements are used. It should also be noted that a shortened notation is used to keep the matrices to a convenient size, with $s_{x}$ denoting the sine of the given orbital element ' $x$ ' and $c_{x}$ denoting the cosine of that element.

Although the perifocal frame and LVLH frame are centered on the Earth and a given spacecraft respectively, the orientation of the principal axes is quite similar.

The only difference is that $\vec{P}_{x}$ is oriented with the orbit's eccentricity vector, while $\vec{L}_{x}$ points to the current position of the spacecraft. As a result, the perifocal frame can be rotated into the LVLH frame by rotating about the z-axis of the perifocal frame through the true anomaly of the spacecraft. Here it should be noted that this can only be used to rotate from perifocal frame for a specific spacecraft to the LVLH frame for that same spacecraft. This rotation can not be used to, for example, rotate between the follower's perifocal frame to the leader's LVLH frame. The rotation matrix that transforms components from the perifocal to the LVLH reference frame is given by

$$
\boldsymbol{C}_{L P}(\nu)=\left[\begin{array}{ccc}
c_{\nu} & s_{\nu} & 0 \\
-s_{\nu} & c_{\nu} & 0 \\
0 & 0 & 1
\end{array}\right]
$$


Rotations may also be performed directly between the ECI and LVLH reference frames by using the two previously defined rotations in series. However, this would constitute a 3-1-3-3 rotation. The two subsequent z-axis rotations, one about the argument of perigee, and the other about the true anomaly, can be combined into a single z-axis rotation about the argument of latitude, defined as the sum of the argument of perigee and the true anomaly. The resulting rotation matrix is

$$
\boldsymbol{C}_{L I}(u, i, \Omega)=\left[\begin{array}{ccc}
c_{\Omega} c_{u}-s_{\Omega} s_{u} c_{i} & s_{\Omega} c_{u}+c_{\Omega} s_{u} c_{i} & s_{u} s_{i} \\
-c_{\Omega} s_{u}-s_{\Omega} c_{u} c_{i} & -s_{\Omega} s_{u}-c_{\Omega} c_{u} c_{i} & c_{u} s_{i} \\
s_{\Omega} s_{i} & -c_{\Omega} s_{i} & c_{i}
\end{array}\right]
$$

It should be noted that all of the previously defined rotations can be inversed to get the rotation in the opposite direction. For example, the inverse of Eq. (2.30) is the rotation from the perifocal to the ECI frame. Therefore, using Eqs. (2.30-2.32), the three important reference frames seen throughout this thesis can be used as needed.

\subsection{Orbital Perturbations}

Thus far, only Keplerian orbits have been discussed. However, numerous forces act on spacecraft in orbit causing significant changes to the actual orbit with time. These forces or accelerations are referred to as orbital perturbations, and the modelling of those perturbations is key to accurately propagating spacecraft motion. The accuracy of the equations for spacecraft formation motion will directly affect the efficiency of using these equations as a guidance system, and therefore the study of orbit perturbations is closely tied with spacecraft formation flying guidance.

Perturbations may be caused by various forces, as any force that acts on the spacecraft other than the gravitational force of the spherical central body about which it orbits constitutes a perturbation. In the case of a spacecraft in orbit around the Earth there are a number of significant perturbations. If a spacecraft is in a low enough orbit the Earth's atmosphere will still be sufficiently thick to cause an appreciable amount of drag. This perturbation is one of the largest for orbits with altitudes less than $1000 \mathrm{~km}$, and the effects continue to some extent beyond this altitude. There are also third-body perturbations, which result from the assumption 
that orbital motion is about a single primary body. In the case of Earth orbits, both the sun and the moon exert an appreciable force on a spacecraft. Another, smaller, perturbation is the force exerted on a spacecraft by solar radiation pressure.

However, arguably the most significant perturbation for Earth orbits results from the fact that the Earth is not a perfectly spherical object. Although the Keplerian orbit motion makes this asssumption, in reality the surface of the Earth varies in height and in mass. This variation causes the Keplerian model of motion to be inaccurate. The non-sphericial gravitational field of the Earth can be modelled by a series of Legendre polynomials and the effects of the non-sphericity can be isolated from the spherical Earth gravity potential to get an equation for the perturbing gravitational force.

By far the most significant component of the non-sphericity results from the fact that the Earth is wider at the equator than at the poles. This oblate spheroid shape, exagerrated for visualization purposes in Fig. 2.4, means that a spacecraft generally experiences higher gravitational forces in its orbit when it passes the equator compared to when it passes the poles. This perturbation, which only depends on latitude, is often given the label $J_{2}$, as it is the second zonal coefficient of the gravitational potential function of the Legendre polynomial describing the Earth's mass distribution. This $J_{2}$ coefficient is approximately three orders of magnitude larger than the next largest coefficients in the gravitational potential function, and has a value of approximately $1.08264 \times 10^{-3}$.

The $J_{2}$ perturbation is of primary interest to this thesis, as accounting for its effects alone significantly improves the accuracy of orbit propagation compared with the unperturbed Keplerian orbit. The perturbing acceleration of $J_{2}$ can be added to the equation for spacecraft acceleration given by Eq. (2.6) as follows

$$
\ddot{\vec{r}}=-\frac{\mu}{r^{3}} \vec{r}+\vec{f}_{J_{2}}
$$

where $\vec{f}_{J_{2}}$ is the perturbing acceleration caused by $J_{2}$. This acceleration can be expressed in terms of components in the ECI reference frame to facilitate its use in the numerical simulator to be defined in the next section. The $\mathrm{x}, \mathrm{y}$ and $\mathrm{z}$ components are as follows [28] 


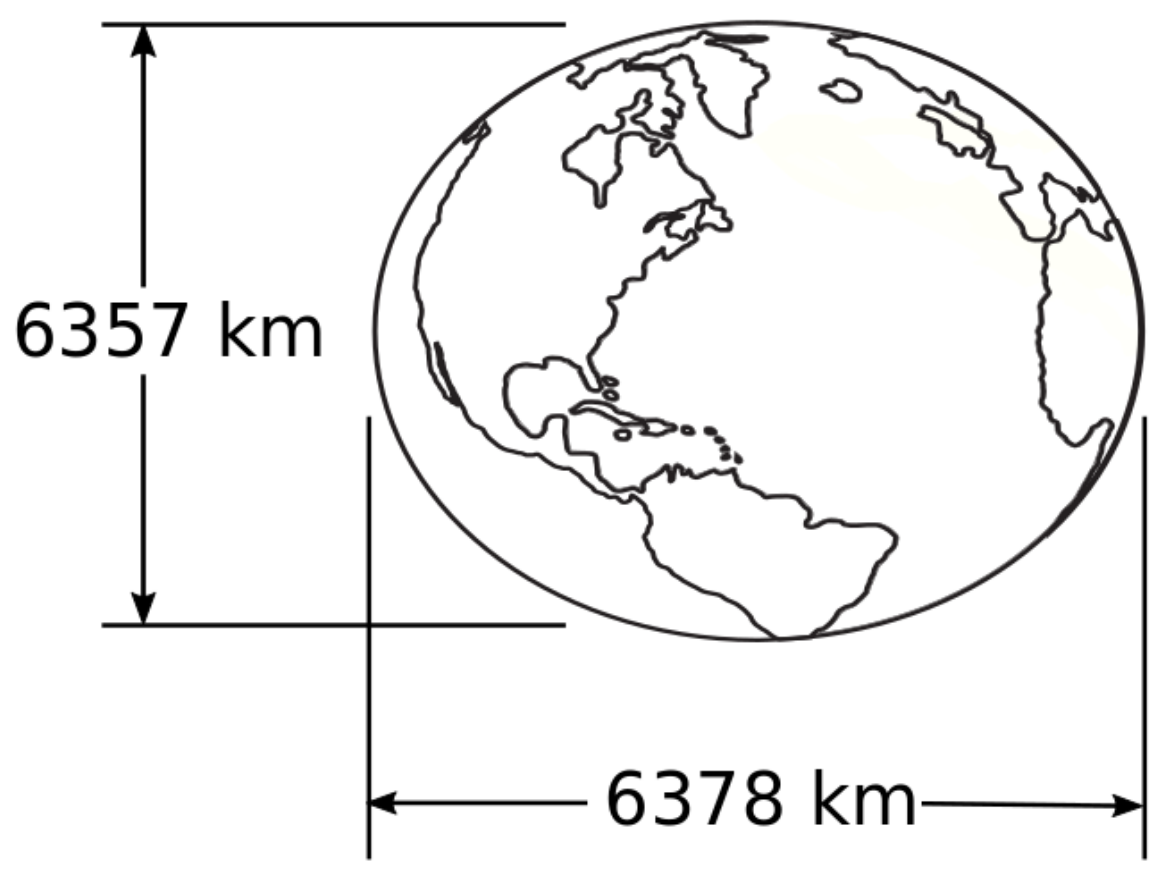

Figure 2.4: Graphical representation of the oblateness of the Earth, which is responsible for the $J_{2}$ perturbation.

$$
\begin{aligned}
& f_{x}=-\frac{\mu x}{r^{3}} J_{2} \frac{3}{2}\left(\frac{R_{E}}{r}\right)^{2}\left(5 \frac{z^{2}}{r^{2}}-1\right) \\
& f_{y}=-\frac{\mu y}{r^{3}} J_{2} \frac{3}{2}\left(\frac{R_{E}}{r}\right)^{2}\left(5 \frac{z^{2}}{r^{2}}-1\right) \\
& f_{z}=-\frac{\mu z}{r^{3}} J_{2} \frac{3}{2}\left(\frac{R_{E}}{r}\right)^{2}\left(3-5 \frac{z^{2}}{r^{2}}\right)
\end{aligned}
$$

where $R_{E}$ is the mean equatorial radius of the Earth, $6378 \mathrm{~km}$, and $x, y$, and $z$ are the magnitude of the components of the position vector in the $\mathrm{x}, \mathrm{y}$ and $\mathrm{z}$ directions.

\subsection{Numerical Simulator}

As mentioned previously, the primary objective of this thesis is to present analytical equations of spacecraft relative motion which are accurate yet computationally simple. To assess the accuracy of the proposed equations, they will be compared with a 
numerical simulator. The simulator created for this purpose evaluates the two-body equation of motion of spacecraft acceleration, Eq. (2.6) and adds the perturbing acceleration resulting from $J_{2}$, calculated using Eqs. 2.34-2.36. This acceleration term is then numerically integrated once to obtain the spacecraft velocity, and a second time to calculate spacecraft postion, which is then fed back into the equations for the accelerations. The numerical integrator is initialized using the initial position and velocity of the spacecraft in the ECI frame. This numerical propagator uses MATLAB with the ODE 45 variable time step solver (with absolute and relative error tolerance set to $\left.1 \times 10^{-} 9\right)$ to propagate accurate spacecraft position and velocity components forward in time in the ECI frame.

Setting up two of these propagators simultaneously, one for the leader spacecraft in formation and one for the follower spacecraft, allows for spacecraft relative motion to be calculated. The leader's position is subtracted from the follower's position to obtain the relative position vector in the ECI frame. Then the leader's position and velocity are converted to a set of orbital elements which are used to calculate the rotation matrix. This rotation matrix is used to rotate this relative position vector into the leader-centered LVLH reference frame. This relative position in the leader's LVLH frame $(\rho)$ can then be compared with the results from the proposed guidance equations to be developed in Chap. 3 of this thesis.

The numerical simulator used throughout the thesis is visualized in Fig. 2.5 below. References are made to the appropriate equations defined previously in this chapter throughout the diagram.

\subsection{Hill-Clohessy-Wiltshire Model for Formation Flying}

Since the objective of this thesis is to develop analytical equations of motion for a spacecraft formation in $J_{2}$-perturbed, eccentric orbits, and to use them for spacecraft formation flying guidance, it is important to not only verify the accuracy of the proposed equations by comparing the calculated relative position with an accurate model of the true relative motion (the numerical simulator), but also to compare their use as a guidance system with another guidance system. It would be impossible to compare 


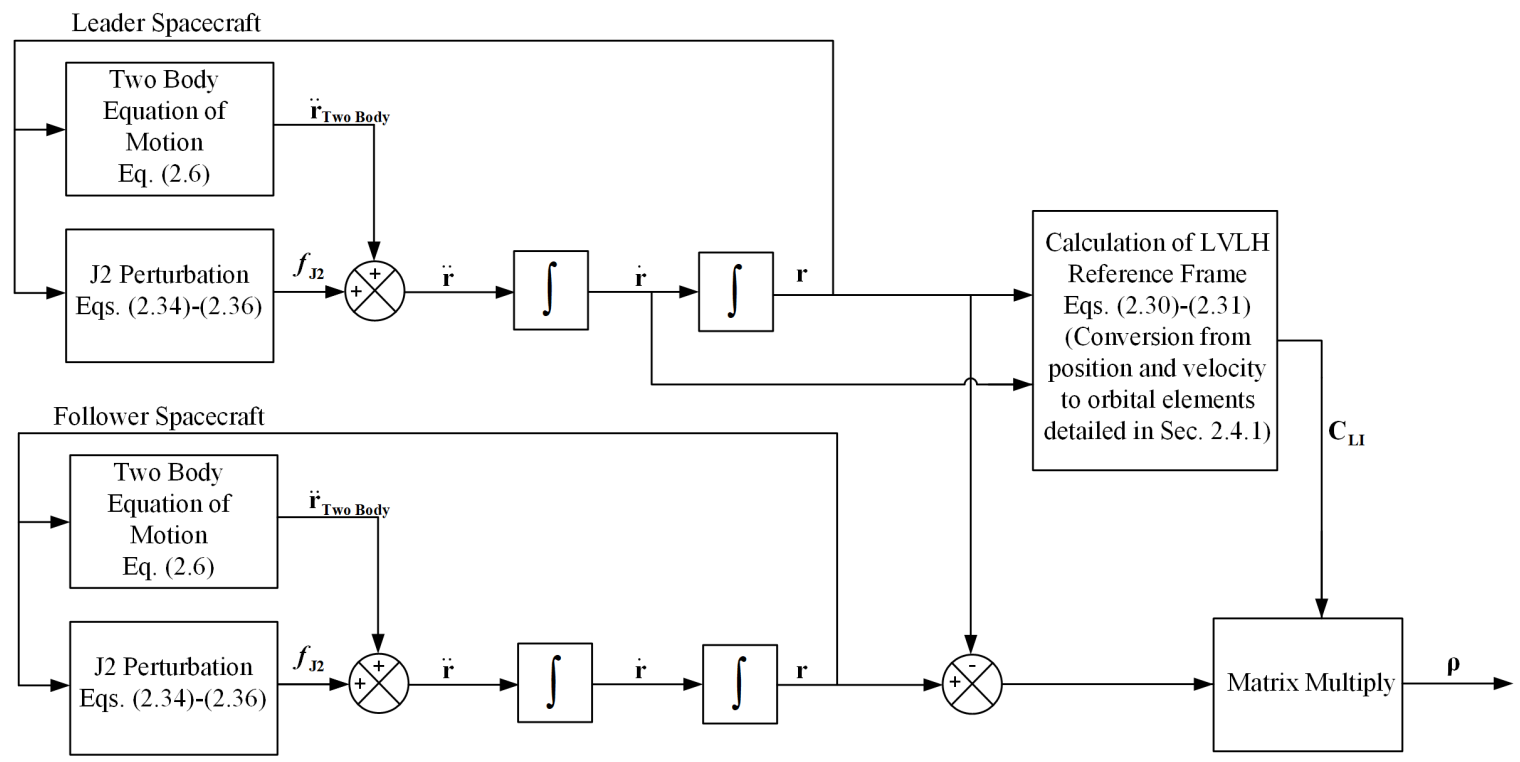

Figure 2.5: Block diagram for the numerical simulator used as the ground truth for spacecraft formation relative position.

with all of the guidance systems in the works outlined in Sec. 1.3. Instead, comparison will be made throughout this work to arguably the most important spacecraft formation flying guidance system used in the literature.

The Hill-Clohessy-Wiltshire (HCW) model for spacecraft formation flying was developed by Clohessy and Witshire [6] based on Hill's work [23] on spacecraft dynamics. The HCW model uses three equations, one in the $\mathrm{x}, \mathrm{y}$ and $\mathrm{z}$ directions in the LVLH reference frame, to describe the position of the follower spacecraft to the leader. This set of three analytical equations is as follows

$$
\begin{gathered}
x(t)=\frac{\dot{x_{0}}}{n} \sin n t-\left(3 x_{0}+\frac{2 \dot{y_{0}}}{n}\right) \cos (n t)+\left(4 x_{0}+\frac{2 \dot{y_{0}}}{n}\right) \\
y(t)=\left(6 x_{0}+\frac{4 \dot{y_{0}}}{n}\right) \sin (n t)+\frac{2 \dot{x_{0}}}{n} \cos n t-\left(6 n x_{0}+3 \dot{y_{0}}\right) t+\left(y_{0}-\frac{2 \dot{x_{0}}}{n}\right) \\
z(t)=z_{0} \cos (n t)+\frac{\dot{z_{0}}}{n} \sin (n t)
\end{gathered}
$$

where $x_{0}, y_{0}$ and $z_{0}$ are the initial relative position components of the formation, $\dot{x}_{0}$, 
$\dot{y}_{0}$ and $\dot{z_{0}}$ are the initial relative velocity components, $n$ is the mean orbital motion of the leader spacecraft, and $t$ is the time elapsed. Thus as long as the semimajor axis and a set of six initial position and velocity components of the relative motion are known, the motion of the formation can be propagated. Through careful choice of the initial conditions, several desirable formations like the along-track or projected circular formation to be set up with ease.

The only disadvantage from using the HCW model for guidance is that a number of simplifying assumptions are made in the derivation of these analytical equations. Specifically, the derivation assumes that the orbits of the spacecraft are circular and Keplerian. As a result, the equations do not accurately predict spacecraft motion in realistic orbits. For more information on the derivation and use of these equations the reader is directed to Clohessy and Wiltshire.[6] 


\section{Chapter 3}

\section{Proposed Equations of Motion}

This chapter discusses the proposed non-linear analytical equations for spacecraft relative motion on $J_{2}$-perturbed eccentric orbits. First, the equations for relative position on Keplerian, eccentric orbits previously derived by Gurfil and Kholshevnikov $[21,22]$ are reviewed. This is followed by the novel work of this thesis extending these equations to work with $J_{2}$-perturbed orbits. Finally, the newly-derived equations are validated in a number of scenarios, proving their accuracy by comparison with the numerical simulator.

\subsection{Introduction}

This chapter describes the derivation and validation of a novel set of analytical equations for spaceraft formation relative motion. Section 3.2: Derivation of Equations of Motion focusses on deriving the equations. In Sec. 3.3: Validation of Proposed Equations, a number of figures comparing the numerical simulator and the proposed equations of motion are presented in an effort to verify their accuracy.

\subsection{Derivation of Equations of Motion}

This section details the methods used to derive a set of nonlinear analytical equations of relative motion which are applicable to $J_{2}$ perturbed eccentric orbits.

\subsubsection{Relative Motion on Keplerian Eccentric Orbits}

Previous work by Gurfil and Kholshevnikov [21, 22] forms a basis for the analytical solution derived herein. In their work, Gurfil and Kholshevnikov proposed a simple method of calculating the relative position of a follower spacecraft with respect to a leader using orbital elements. This allows the equations of relative motion to remain 
accurate for arbitrarily large values of eccentricity. To do so, consecutive rotations and a translation are applied to obtain an analytical expression for $\boldsymbol{\rho}$, defined as the components of the relative position vector in $\mathcal{F}_{L}$, that is

$$
\boldsymbol{\rho}=\boldsymbol{r}_{L}-\boldsymbol{r}_{L}^{\prime}
$$

where $\boldsymbol{r}_{L}$ and $\boldsymbol{r}_{L}^{\prime}$ respectively denote the components of the follower and leader spacecraft position vector expressed in $\mathcal{F}_{L}$. The first step in calculating $\boldsymbol{\rho}$ is to transform the components of the follower position vector from $\mathcal{F}_{P}$ to $\mathcal{F}_{I}$ using $\boldsymbol{C}_{I P}(\omega, i, \Omega)$, the 3-1-3 rotation sequence given by

$$
\boldsymbol{C}_{I P}(\omega, i, \Omega)=\left[\begin{array}{ccc}
c_{\Omega} c_{\omega}-s_{\Omega} s_{\omega} c_{i} & -c_{\Omega} s_{\omega}-s_{\Omega} c_{\omega} c_{i} & s_{\Omega} s_{i} \\
s_{\Omega} c_{\omega}+c_{\Omega} s_{\omega} c_{i} & -s_{\Omega} s_{\omega}-c_{\Omega} c_{\omega} c_{i} & -c_{\Omega} s_{i} \\
s_{\omega} s_{i} & c_{\omega} s_{i} & c_{i}
\end{array}\right]
$$

where $\Omega, i$, and $\omega$ respectively denote the right ascension of the ascending node (RAAN), inclination, and argument of perigee of the follower spacecraft. The next step is to transform the components of the follower position vector from $\mathcal{F}_{I}$ to $\mathcal{F}_{P^{\prime}}$ using $\boldsymbol{C}_{P^{\prime} I}\left(\omega^{\prime}, i^{\prime}, \Omega^{\prime}\right)$ given by

$$
C_{P^{\prime} I}\left(\omega^{\prime}, i^{\prime}, \Omega^{\prime}\right)=\left[\begin{array}{ccc}
c_{\Omega^{\prime}} c_{\omega^{\prime}}-s_{\Omega^{\prime}} s_{\omega^{\prime}} c_{i^{\prime}} & s_{\Omega^{\prime}} c_{\omega^{\prime}}+c_{\Omega^{\prime}} s_{\omega^{\prime}} c_{i^{\prime}} & s_{\omega^{\prime}} s_{i^{\prime}} \\
-c_{\Omega^{\prime}} s_{\omega^{\prime}}-s_{\Omega^{\prime}} c_{\omega^{\prime}} c_{i^{\prime}} & -s_{\Omega^{\prime}} s_{\omega^{\prime}}-c_{\Omega^{\prime}} c_{\omega^{\prime}} c_{i^{\prime}} & c_{\omega^{\prime}} s_{i^{\prime}} \\
s_{\Omega^{\prime}} s_{i^{\prime}} & -c_{\Omega^{\prime}} s_{i^{\prime}} & c_{i^{\prime}}
\end{array}\right]
$$

where $\Omega^{\prime}, i^{\prime}$, and $\omega^{\prime}$ denote the leader's RAAN, inclination, and argument of perigee, respectively. Then, the components of the follower position vector are rotated from $\mathcal{F}_{P^{\prime}}$ to $\mathcal{F}_{L}$ with $\boldsymbol{C}_{L P^{\prime}}\left(\nu^{\prime}\right)$

$$
\boldsymbol{C}_{L P^{\prime}}\left(\nu^{\prime}\right)=\left[\begin{array}{ccc}
c_{\nu^{\prime}} & s_{\nu^{\prime}} & 0 \\
-s_{\nu^{\prime}} & c_{\nu^{\prime}} & 0 \\
0 & 0 & 1
\end{array}\right]
$$

where $\nu^{\prime}$ denotes the true anomaly of the leader spaceraft. Finally, a translation is applied, such that 


$$
\boldsymbol{\rho}=\boldsymbol{C}_{L P^{\prime}}\left(\nu^{\prime}\right) \boldsymbol{C}_{P^{\prime} I}\left(\omega^{\prime}, i^{\prime}, \Omega^{\prime}\right) \boldsymbol{C}_{I P}(\omega, i, \Omega) \boldsymbol{r}_{P}-\boldsymbol{r}_{L}^{\prime}
$$

where $\boldsymbol{r}_{P}$ and $\boldsymbol{r}_{L}^{\prime}$ represents the components of the follower's position vector in $\mathcal{F}_{P}$, and the components of the leader's position vector in $\mathcal{F}_{L}$, respectively given by

$$
\begin{gathered}
\boldsymbol{r}_{P}=\left[\begin{array}{lll}
r \cos \nu & r \sin \nu & 0
\end{array}\right]^{T} \\
\boldsymbol{r}_{L}^{\prime}=\left[\begin{array}{lll}
r^{\prime} & 0 & 0
\end{array}\right]^{T}
\end{gathered}
$$

where $\nu$ denotes the true anomaly of the follower spaceraft, and where the orbit radii, $r$ and $r^{\prime}$, are obtained from the orbit equation

$$
\begin{gathered}
r=\frac{a\left(1-e^{2}\right)}{1+e \cos \nu} \\
r^{\prime}=\frac{a^{\prime}\left(1-e^{\prime 2}\right)}{1+e^{\prime} \cos \nu^{\prime}}
\end{gathered}
$$

with $a$ and $e$ denoting the semimajor axis and eccentricity of the follower, and $a^{\prime}$ and $e^{\prime}$ denoting the semimajor axis and eccentricity of the leader spacecraft. Note that Eq. (3.5) can be written component-wise and further simplified through the use of relative orbital elements. For more details, the readers are referred to the work of Gurfil and Kholshevnikov.[21, 22]

\subsubsection{Relative Motion on $J_{2}$-Perturbed Eccentric Orbits}

The previous equations use constant orbital elements to calculate the position of a follower spacecraft in $\mathcal{F}_{L}$. Using constant orbital elements is a good approximation for the solution, and may be desirable as it greatly simplifies the computations for predicting the relative motion in the LVLH frame. The effectivenes of these existing equations was validated at a variety of eccentricity values, ranging from 0 to 0.8. The results are provided in Fig. 8.1 (a). This figure clearly demonstrates that the equations are effective at predicting relative motion in eccentric Keplerian orbits. However, perturbing accelerations will have a significant effect on the relative 
motion of the spacecraft. The most dominant perturbation, and therefore the most important perturbation to consider, is the $J_{2}$ gravitational perturbation. One notable consequence of $J_{2}$ on the relative motion is a drift between both spacecraft along the $\vec{L}_{y}$ direction. Figure 3.1 (b) shows an example of this drifting motion for a typical spacecraft formation. This figure also shows, in part (a), that the accuracy of the Gurfil and Kholshevnikov analytical solution drops significantly once the orbit is $J_{2}$-perturbed.
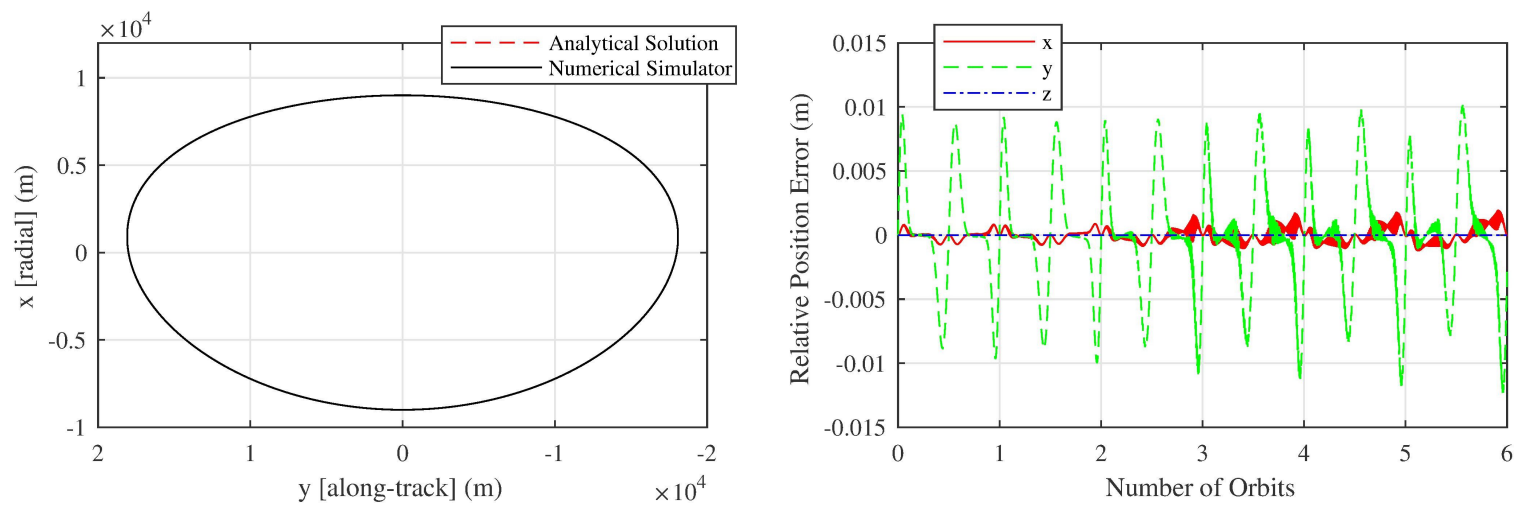

(a) Without J2 Perturbation
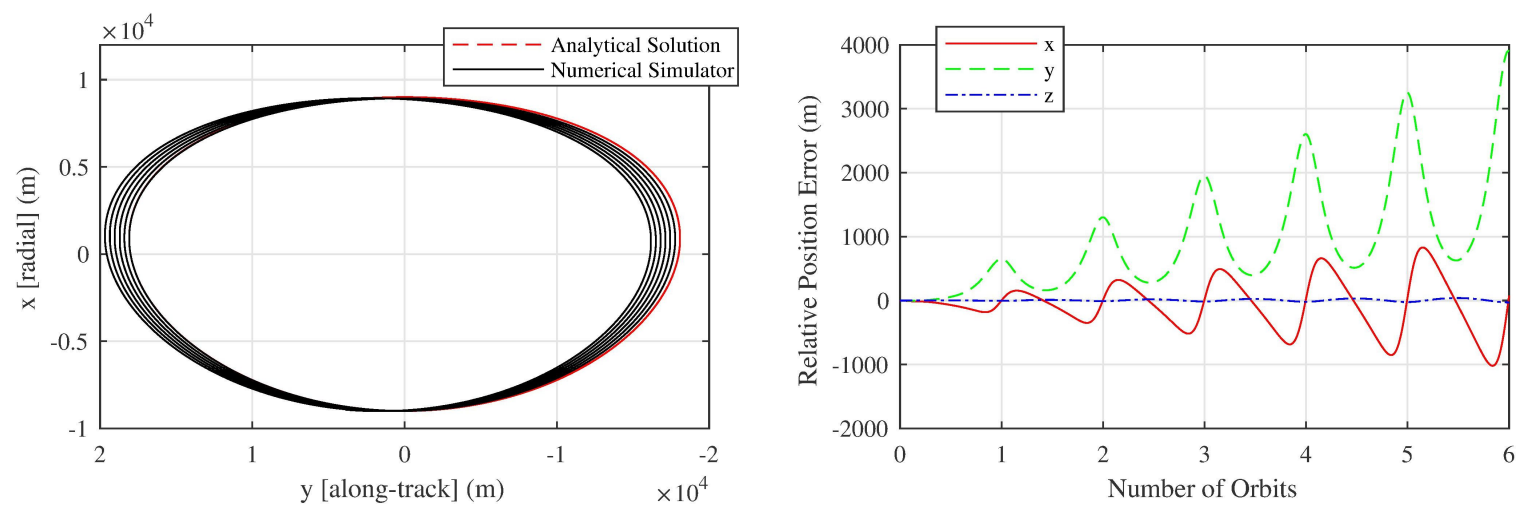

(b) With J2 Perturbation

Figure 3.1: Figure showing the formation drifting effect caused by J2; in-plane elliptical formation with $a^{\prime}=9000 \mathrm{~km}$ and $e^{\prime}=0.2$.

It should be noted, however, that Eq. (3.5) represents an exact solution for the relative position of the follower spacecraft at any given time, assuming that the orbital elements for each spacecraft used in the equation are accurate. Therefore, if accurate $J_{2}$-perturbed osculating orbital elements are used instead of constant ones, Eq. (3.5) would then result in an accurate solution for the relative position between 
both spacecraft under the influence of $J_{2}$. In other words, $J_{2}$-perturbed osculating orbital elements for each spacecraft can be used with Eq. (3.5) to accurately predict the components of the relative position vector in LVLH, i.e., $\boldsymbol{\rho}$.

The $J_{2}$-perturbed osculating orbital elements, $[a, e, i, \omega, \Omega, M]^{T}$, can be approximated by summing mean orbital elements with the $J_{2}$-induced short-periodic variations, as follows

$$
\begin{aligned}
& a=\bar{a}+\Delta a_{s p} \\
& e=\bar{e}+\Delta e_{s p} \\
& i=\bar{i}+\Delta i_{s p} \\
& \omega=\bar{\omega}+\Delta \omega_{s p} \\
& \Omega=\bar{\Omega}+\Delta \Omega_{s p} \\
& M=\bar{M}+\Delta M_{s p}
\end{aligned}
$$

where $\left[\Delta a_{s p}, \Delta e_{s p}, \Delta i_{s p}, \Delta \omega_{s p}, \Delta \Omega_{s p}, \Delta M_{s p}\right]^{T}$, denote the short-periodic variations of the orbital elements, and where the mean orbital elements, $[\bar{a}, \bar{e}, \bar{i}, \bar{\omega}, \bar{\Omega}, \bar{M}]^{T}$, can be described as the initial mean orbital elements, denoted by $\left[\bar{a}_{0}, \bar{e}_{0}, \bar{i}_{0}, \bar{\omega}_{0}, \bar{\Omega}_{0}, \bar{M}_{0}\right]^{T}$, summed with the secular variations due to $J_{2}$

$$
\begin{aligned}
& \bar{a}=\bar{a}_{0} \\
& \bar{e}=\bar{e}_{0} \\
& \bar{i}=\bar{i}_{0} \\
& \bar{\omega}=\bar{\omega}_{0}+\dot{\bar{\omega}} t \\
& \bar{\Omega}=\bar{\Omega}_{0}+\dot{\bar{\Omega}} t \\
& \bar{M}=\bar{M}_{0}+\dot{\bar{M}} t
\end{aligned}
$$

where $t$ is the elasped time since $t_{0}$. It has been shown that, as a result of the $J_{2}$ perturbation, the orbital elements $a, e$, and $i$ have zero secular change, while the remaining elements do have some nonzero secular rate of change, denoted by $[\dot{\bar{\omega}}, \dot{\bar{\Omega}}, \dot{\bar{M}}]^{T}[27]$ 


$$
\begin{gathered}
\dot{\bar{\omega}}=\frac{1}{2} \bar{n} J_{2}\left(\frac{R_{e}}{\bar{p}}\right)^{2}\left(4-5 \sin ^{2} \bar{i}\right) \\
\dot{\bar{\Omega}}=-\frac{3}{2} \bar{n} J_{2}\left(\frac{R_{e}}{\bar{p}}\right)^{2} \cos \bar{i} \\
\dot{\bar{M}}=\bar{n}+\frac{3}{2} \bar{n} J_{2}\left(\frac{R_{e}}{\bar{a}}\right)^{2} \frac{1}{\left(1-\bar{e}^{2}\right)^{3 / 2}}\left(1-\frac{3}{2} \sin ^{2} \bar{i}\right)
\end{gathered}
$$

where the mean orbital motion and semilatus rectum calculated with the mean semimajor axis and eccentricity, denoted as $\bar{n}$ and $\bar{p}$, respectively, are given by

$$
\begin{gathered}
\bar{n}=\sqrt{\frac{\mu}{\bar{a}^{3}}} \\
\bar{p}=\bar{a}\left(1-\bar{e}^{2}\right)
\end{gathered}
$$

Now that the mean orbital elements are fully defined for each spacecraft, the short periodic variations of each element must be calculated, as follows [27]

$$
\begin{aligned}
\Delta a_{s p}= & \frac{J_{2} R_{e}^{2}}{a}\left[\left(\frac{a}{r}\right)^{3}-\frac{1}{\left(1-e^{2}\right)^{3 / 2}}+\left\{-\left(\frac{a}{r}\right)^{3}+\frac{1}{\left(1-e^{2}\right)^{3 / 2}}+\left(\frac{a}{r}\right)^{3} \cos (2 \omega+2 \nu)\right\} \frac{3 \sin ^{2} i}{2}\right] \\
\Delta e_{s p}= & \frac{J_{2} R_{e}^{2}}{4}\left[\frac{-2}{a^{2} e \sqrt{1-e^{2}}}+\frac{2 a\left(1-e^{2}\right)}{e r^{3}}+\left\{\frac{3}{a^{2} e \sqrt{1-e^{2}}}-\frac{3 a\left(1-e^{2}\right)}{e r^{3}}\right.\right. \\
& -\frac{3\left(1-e^{2}\right) \cos (\nu+2 \omega)}{p^{2}}-\frac{3 \cos (2 \nu+2 \omega)}{a^{2} e\left(1-e^{2}\right)}+\frac{3 a\left(1-e^{2}\right) \cos (2 \nu+2 \omega)}{e r^{3}} \\
& \left.\left.-\frac{\left(1-e^{2}\right) \cos (3 \nu+2 \omega)}{p^{2}}\right\} \sin ^{2} i\right]
\end{aligned}
$$

$$
\Delta i_{s p}=\frac{J_{2} R_{e}^{2} \sin (2 i)}{8 p^{2}}[3 \cos (2 \omega+2 \nu)+3 e \cos (2 \omega+\nu)+e \cos (2 \omega+3 \nu)]
$$


$\Delta \Omega_{s p}=\frac{J_{2} R_{e}^{2} \cos (i)}{4 p^{2}}[6(\nu-M+e \sin \nu)-3 \sin (2 \omega+2 \nu)-3 e \sin (2 \omega+\nu)-e \sin (2 \omega+3 \nu)]$

$$
\begin{aligned}
\Delta \omega_{s p}= & \frac{3 J_{2} R_{e}^{2}}{2 p^{2}}\left[\left(2-\frac{5}{2} \sin ^{2} i\right)(\nu-M+e \sin \nu)\right. \\
& +\left(1-\frac{3}{2} \sin ^{2} i\right)\left\{\frac{1}{e}\left(1-\frac{1}{4} e^{2}\right) \sin \nu+\frac{1}{2} \sin (2 \nu)+\frac{e}{12} \sin (3 \nu)\right\} \\
& -\frac{1}{e}\left\{\frac{1}{4} \sin ^{2} i+\left(\frac{1}{2}-\frac{15}{16} \sin ^{2} i\right) e^{2}\right\} \sin (\nu+2 \omega)+\frac{e}{16} \sin ^{2} i \sin (\nu-2 \omega) \\
& -\frac{1}{2}\left(1-\frac{5}{2} \sin ^{2} i\right) \sin (2 \nu+2 \omega) \\
& +\frac{1}{e}\left\{\frac{7}{12} \sin ^{2} i-\frac{1}{6}\left(1-\frac{19}{8} \sin ^{2} i\right) e^{2}\right\} \sin (3 \nu+2 \omega) \\
& \left.+\frac{3}{8} \sin ^{2} i \sin (4 \nu+2 \omega)+\frac{e}{16} \sin ^{2} i \sin (5 \nu+2 \omega)\right]
\end{aligned}
$$

$$
\begin{aligned}
\Delta M_{s p}= & \frac{3 J_{2} R_{e}^{2} \sqrt{1-e^{2}}}{2 e p^{2}}\left[-\left(1-\frac{3}{2} \sin ^{2}(i)\right)\left\{\left(1-\frac{e^{2}}{4}\right) \sin \nu+\frac{e}{2} \sin (2 \nu)+\frac{e^{2}}{12} \sin (3 \nu)\right\}\right. \\
& +\sin ^{2} i\left\{\frac{1}{4}\left(1+\frac{5}{4} e^{2}\right) \sin (\nu+2 \omega)-\frac{e^{2}}{16} \sin (\nu-2 \omega)\right. \\
& \left.\left.-\frac{7}{12}\left(1-\frac{e^{2}}{28}\right) \sin (3 \nu+2 \omega)-\frac{3 e}{8} \sin (4 \nu+2 \omega)-\frac{e^{2}}{16} \sin (5 \nu+2 \omega)\right\}\right]
\end{aligned}
$$

The true anomaly, $\nu$, is also required to make use of the previously-defined equations. This calculation must obviously account for the $J_{2}$ perturbation, as the perturbation will have an effect on the true anomaly. In their paper, Gurfil and Kholshevnikov $[21,22]$ proposed to numerically integrate an expression for the time derivative of the true anomaly. However, doing so is undesirable in terms of computational efficiency. Fortunately, $\nu$ can be obtained iteratively from the mean anomaly and the orbit eccentricity through the relations

$$
E=M+e \sin (M+e \sin (M+e \sin (M+\cdots+e \sin (M))))
$$




$$
\begin{gathered}
\cos \nu=\frac{\cos E-e}{1-e \cos E} \\
\sin \nu=\frac{\sqrt{1-e^{2}} \sin E}{1-e \cos E} \\
\nu=\tan ^{-1} \frac{\sin \nu}{\cos \nu}
\end{gathered}
$$

where $E$ denotes the eccentric anomaly. The number of terms in Eq. (3.23) is selected in accordance with the desired accuracy and is strictly a function of the magnitude of the eccentricity. For higher eccentricity values more terms will be needed to get good accuracy from the relation.

\subsubsection{Simplification of Proposed Equations}

For ease of use as a guidance law, the relative motion solution should be described in a set of three analytical equations; one for each direction of the local-vertical-localhorizontal reference frame $\mathcal{F}_{L}$. This is accomplished by defining constants based on the rotation matrices previously defined. Equation (3.27) shows this set of three analytical equations, one for the $\vec{L}_{x}, \vec{L}_{y}$, and $\vec{L}_{z}$ directions

$$
\begin{aligned}
& x(t)=r \cos (\nu)\left(k_{1}^{\prime} k_{1}+k_{2}^{\prime} k_{4}+k_{3}^{\prime} k_{7}\right)+r \sin (\nu)\left(k_{1}^{\prime} k_{2}+k_{2}^{\prime} k_{5}+k_{3}^{\prime} k_{8}\right)-r^{\prime} \\
& y(t)=r \cos (\nu)\left(k_{4}^{\prime} k_{1}+k_{5}^{\prime} k_{4}+k_{6}^{\prime} k_{7}\right)+r \sin (\nu)\left(k_{4}^{\prime} k_{2}+k_{5}^{\prime} k_{5}+k_{6}^{\prime} k_{8}\right) \\
& z(t)=r \cos (\nu)\left(k_{7}^{\prime} k_{1}+k_{8}^{\prime} k_{4}+k_{9}^{\prime} k_{7}\right)+r \sin (\nu)\left(k_{7}^{\prime} k_{2}+k_{8}^{\prime} k_{5}+k_{9}^{\prime} k_{8}\right)
\end{aligned}
$$

where $x, y$ and $z$ are the components of the relative position vector, that is

$$
\boldsymbol{\rho}=\left[\begin{array}{l}
x \\
y \\
z
\end{array}\right]
$$

The constants in Eq. 3.27, denoted by k's, are herein defined as 


$$
\begin{aligned}
k_{1} & =\cos (\Omega) \cos (\omega)-\sin (\Omega) \sin (\omega) \cos (i) \\
k_{2} & =-\cos (\Omega) \sin (\omega)-\sin (\Omega) \cos (\omega) \cos (i) \\
k_{3} & =\sin (\Omega) \sin (i) \\
k_{4} & =\sin (\Omega) \cos (\omega)+\cos (\Omega) \sin (\omega) \cos (i) \\
k_{5} & =-\sin (\Omega) \sin (\omega)-\cos (\Omega) \cos (\omega) \cos (i) \\
k_{6} & =-\cos (\Omega) \sin (i) \\
k_{7} & =\sin (\omega) \sin (i) \\
k_{8} & =\cos (\omega) \cos (i) \\
k_{9} & =\cos (i) \\
k_{1}^{\prime} & =\cos \left(\Omega^{\prime}\right) \cos \left(u^{\prime}\right)-\sin \left(\Omega^{\prime}\right) \sin \left(u^{\prime}\right) \cos \left(i^{\prime}\right) \\
k_{2}^{\prime} & =\sin \left(\Omega^{\prime}\right) \cos \left(u^{\prime}\right)+\cos \left(\Omega^{\prime}\right) \sin \left(u^{\prime}\right) \cos \left(i^{\prime}\right) \\
k_{3}^{\prime} & =\sin \left(u^{\prime}\right) \sin \left(i^{\prime}\right) \\
k_{4}^{\prime} & =-\cos \left(\Omega^{\prime}\right) \sin \left(u^{\prime}\right)-\sin \left(\Omega^{\prime}\right) \cos \left(u^{\prime}\right) \cos \left(i^{\prime}\right) \\
k_{5}^{\prime} & =-\sin \left(\Omega^{\prime}\right) \sin \left(u^{\prime}\right)-\cos \left(\Omega^{\prime}\right) \cos \left(u^{\prime}\right) \cos \left(i^{\prime}\right) \\
k_{6}^{\prime} & =\cos \left(u^{\prime}\right) \sin \left(i^{\prime}\right) \\
k_{7}^{\prime} & =\sin \left(\Omega^{\prime}\right) \sin \left(i^{\prime}\right) \\
k_{8}^{\prime} & =-\cos \left(\Omega^{\prime}\right) \sin \left(i^{\prime}\right) \\
k_{9}^{\prime} & =\cos \left(i^{\prime}\right)
\end{aligned}
$$

Note that $u$ is the argument of latitude, defined as the sum of $\nu$ and $\omega$, which allows the matrices from Eqs. (3.3) and (3.4) to be combined as follows

$$
\boldsymbol{C}_{L I}\left(u^{\prime}, i^{\prime}, \Omega^{\prime}\right)=\left[\begin{array}{ccc}
c_{\Omega^{\prime}} c_{u^{\prime}}-s_{\Omega^{\prime}} s_{u^{\prime}} c_{i^{\prime}} & s_{\Omega^{\prime}} c_{u^{\prime}}+c_{\Omega^{\prime}} s_{u^{\prime}} c_{i^{\prime}} & s_{u^{\prime}} s_{i^{\prime}} \\
-c_{\Omega^{\prime}} s_{u^{\prime}}-s_{\Omega^{\prime}} c_{u^{\prime}} c_{i^{\prime}} & -s_{\Omega^{\prime}} s_{u^{\prime}}-c_{\Omega^{\prime}} c_{u^{\prime}} c_{i^{\prime}} & c_{u^{\prime}} s_{i^{\prime}} \\
s_{\Omega^{\prime}} s_{i^{\prime}} & -c_{\Omega^{\prime}} s_{i^{\prime}} & c_{i^{\prime}}
\end{array}\right]
$$

Equation (3.27), along with definitions of constants previously defined, are a more 
compact way of expressing the Gurfil and Kholshevnikov solution presented previously. This is an exact solution for the relative position of a follower spacecraft in $\mathcal{F}_{L}$ as long as the orbital elements used in the calculation of this relative position are accurate. Assuming that the orbital elements are constant leads to an exact solution for relative position on Keplerian orbits. The same equations can be applied with the $J_{2}$-perturbed osculating orbital elements defined in the previous subsection to obtain a relative position solution which accounts for the $J_{2}$ perturbation.

It should be noted that using Eq. (3.27) in this way implies that the expressions for $k$ 's in Eqs. (3.29) and (3.30) are not truly constants, but functions of time. This means that they must be calculated at a given time to find the relative position, but they still help to simplify the equations of relative position. The use of $J_{2}$-perturbed osculating orbital elements implies that the orbital elements used to calculate each constant in Eqs. (3.29) and (3.30) are also functions of time, but the $(t)$ notation is omitted here to aid in the readability of the expressions.

Another method for presenting the equations of motion is to substitute the rotations from the follower's perifocal to the inertial frame, and the inertial to the leader's perifocal frame into a single rotation. These two rotations can be accomplished through the use of a single 3-1-3 rotation sequence analagous to the rotation from a perifocal to the inertial reference frame. In other words, the following matrix may replace the multiplication of the matrices given in Eqs. (3.2) and (3.3)

$$
\boldsymbol{C}_{L P}(\omega, i, \Omega)=\left[\begin{array}{ccc}
c_{\Omega^{*}} c_{\omega^{*}}-s_{\Omega^{*}} s_{\omega^{*}} c_{i^{*}} & -c_{\Omega^{*}} s_{\omega^{*}}-s_{\Omega^{*}} c_{\omega^{*}} c_{i^{*}} & s_{\Omega^{*}} s_{i^{*}} \\
s_{\Omega^{*}} c_{\omega^{*}}+c_{\Omega^{*}} s_{\omega^{*}} c_{i^{*}} & -s_{\Omega^{*}} s_{\omega^{*}}-c_{\Omega^{*}} c_{\omega^{*}} c_{i^{*}} & -c_{\Omega^{*}} s_{i^{*}} \\
s_{\omega^{*}} s_{i^{*}} & c_{\omega^{*}} s_{i^{*}} & c_{i^{*}}
\end{array}\right]
$$

with $\Omega^{*}, \omega^{*}$ and $i^{*}$ being defined as the relative right ascension of ascending node, relative argument perigee and relative inclination respectively. The relative RAAN is the angle between the eccentricity vector of the leader spacecraft and the relative line of nodes (RLON), defined as the line of intersection of the orbital planes of the two spacecraft. The relative argument of perigee is defined as the angle between the relative line of nodes and the follower spacecrafts eccentricity vector. Finally, the 
relative inclination is the angle between the orbit normal vector of each spacecraft. These relative orbital elements are represented graphically in Fig. 3.2.

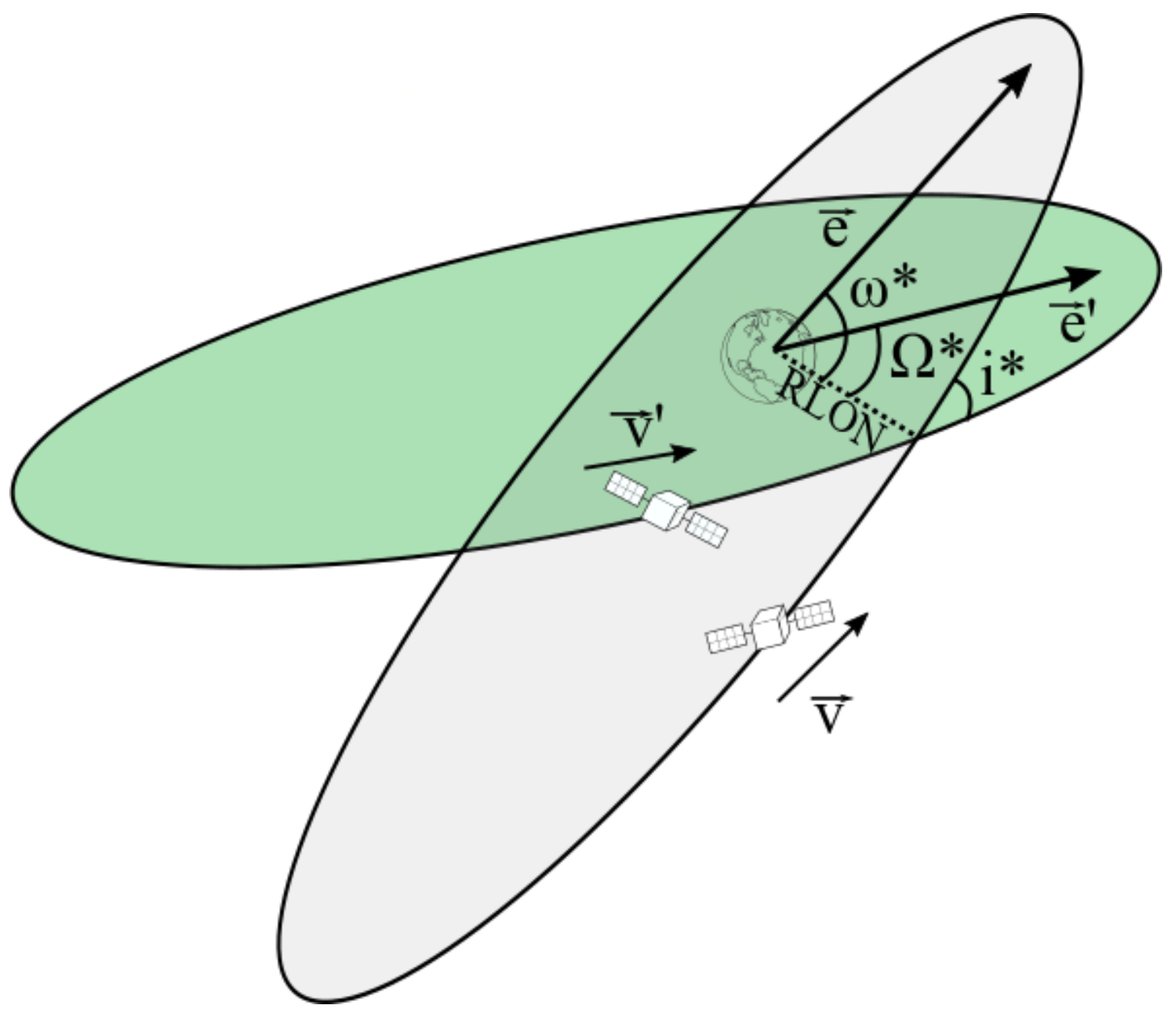

Figure 3.2: Diagram showing the relative orbital elements, the green ellipse represents the orbit plane of the leader, and the grey ellipse is the orbit plane of the follower.

Using the relative orbital elements Eq. (3.5) can then be rewritten as follows.

$$
\boldsymbol{\rho}=\boldsymbol{C}_{L P^{\prime}}\left(\nu^{\prime}\right) \boldsymbol{C}_{I P}\left(\omega^{*}, i^{*}, \Omega^{*}\right) \boldsymbol{r}_{P}-\boldsymbol{r}_{L}^{\prime}
$$

Unit vectors expressing the direction of the eccentricty vector and orbit normal vector can be defined using Eqs. (3.2) and (3.3) resulting in the following definitions 


$$
\begin{gathered}
\boldsymbol{e}_{I}^{\prime}=\left[\begin{array}{c}
\cos \left(\Omega^{\prime}\right) \cos \left(\omega^{\prime}\right)-\sin \left(\Omega^{\prime}\right) \sin \left(\omega^{\prime}\right) \cos \left(i^{\prime}\right) \\
\sin \left(\Omega^{\prime}\right) \cos \left(\omega^{\prime}\right)+\cos \left(\Omega^{\prime}\right) \sin \left(\omega^{\prime}\right) \cos \left(i^{\prime}\right) \\
\sin \left(i^{\prime}\right) \sin \left(\omega^{\prime}\right)
\end{array}\right] \\
\boldsymbol{n}_{I}^{\prime}=\left[\begin{array}{c}
\sin \left(\Omega^{\prime}\right) \sin \left(i^{\prime}\right) \\
-\cos \left(\Omega^{\prime}\right) \sin \left(i^{\prime}\right) \\
\cos \left(i^{\prime}\right)
\end{array}\right]
\end{gathered}
$$

and similarly for the follower spacecraft. The relative line of nodes is then given by the following cross product

$$
\boldsymbol{R L O N} \boldsymbol{N}_{I}=\boldsymbol{n}_{I}^{\prime \times} \boldsymbol{n}_{I}
$$

where \{\}$^{\times}$denotes the skew-symmetric operation. The relative line of nodes is not necessarily a unit vector, but should be made so by dividing the components by the magnitude. Finally the relative orbital elements are given by the following three equations

$$
\begin{gathered}
\Omega^{*}=\cos ^{-1}\left(\boldsymbol{e}_{I}^{\prime} \cdot \boldsymbol{R L O N} \boldsymbol{N}_{I}\right) \\
\omega^{*}=\cos ^{-1}\left(\boldsymbol{e}_{I} \cdot \boldsymbol{R L} \boldsymbol{O} \boldsymbol{N}_{I}\right) \\
i^{*}=\cos ^{-1}\left(\boldsymbol{n}_{I} \cdot \boldsymbol{n}_{I}^{\prime}\right)
\end{gathered}
$$

Separating Eq. (3.33) into $x, y$, and $z$ components in the $\vec{L}_{x}, \vec{L}_{y}$, and $\vec{L}_{z}$ directions allows the three analytical equations to be written as

$$
\begin{aligned}
& x(t)=r \cos (\nu)\left(\cos \left(\nu^{\prime}\right) k_{1}^{*}+\sin \left(\nu^{\prime}\right) k_{3}^{*}\right)+r \sin (\nu)\left(-\cos \left(\nu^{\prime}\right) k_{2}^{*}-\sin \left(\nu^{\prime}\right) k_{4}^{*}\right)-r^{\prime} \\
& y(t)=r \cos (\nu)\left(-\sin \left(\nu^{\prime}\right) k_{1}^{*}+\cos \left(\nu^{\prime}\right) k_{3}^{*}\right)+r \sin (\nu)\left(\sin \left(\nu^{\prime}\right) k_{2}^{*}-\cos \left(\nu^{\prime}\right) k_{4}^{*}\right) \\
& z(t)=r \cos (\nu) k_{5}^{*}+r \sin (\nu) k_{6}^{*}
\end{aligned}
$$

where the $k^{*}$ values are defined as follows 


$$
\begin{aligned}
& k_{1}^{*}(t)=\left(\cos \left(\Omega^{*}\right) \cos \left(\omega^{*}\right)-\sin \left(\Omega^{*}\right) \sin \left(\omega^{*}\right) \cos \left(i^{*}\right)\right) \\
& k_{2}^{*}(t)=\left(-\cos \left(\Omega^{*}\right) \sin \left(\omega^{*}\right)-\sin \left(\Omega^{*}\right) \cos \left(\omega^{*}\right) \cos \left(i^{*}\right)\right) \\
& k_{3}^{*}(t)=\left(\sin \left(\Omega^{*}\right) \cos \left(\omega^{*}\right)+\cos \left(\Omega^{*}\right) \sin \left(\omega^{*}\right) \cos \left({ }^{*} i\right)\right) \\
& k_{4}^{*}(t)=\left(-\sin \left(\Omega^{*}\right) \sin \left(\omega^{*}\right)+\cos \left(\Omega^{*}\right) \cos \left(\omega^{*}\right) \cos \left(i^{*}\right)\right) \\
& k_{5}^{*}(t)=\sin \left(\omega^{*}\right) \sin \left(i^{*}\right) \\
& k_{6}^{*}(t)=\cos \left(\omega^{*}\right) \sin \left(i^{*}\right)
\end{aligned}
$$

Equation 3.40 has the advantage of relying less heavily on the definition of constants by making use of relative orbital elements, which are properties of the orbits of the two spacecraft in formation. It may therefore provide more insight into the motion of the spacecraft (when compared to Eq. (3.27)) by being based on these physical properties. It should be noted that these two simplified versions of the proposed equations of motion are equivalent, and will give the same accuracy when used. This was verified through MATLAB situations. Moving forward, any results and practical application scenarios of the proposed equations described in this thesis use Eq. (3.27), as this set of equations requires fewer computations compared to Eq. (3.40).

\subsection{Verification of Proposed Equations}

To verify the accuracy of the newly-developed method of calculating the relative position between two spacecraft, numerical simulations were performed in MATLAB. The results obtained with the analytical solution provided in this thesis were compared to a numerical simulator that integrates the exact, nonlinear differential equations of motion in $\mathcal{F}_{I}$. Specifically, the simulator integrates, for each spacecraft, the inertial two-body equations of motion to which the $J_{2}$ inertial perturbing acceleration was added. The position vectors of both spacecraft are then transformed into $\mathcal{F}_{L}$. Finally, the leader position vector is subtracted from the follower position vector to obtain $\boldsymbol{\rho}$, the components of the exact relative position vector in $\mathcal{F}_{L}$. The simulator was initialized for both spacecraft using the initial osculating orbital elements which were converted into components of the initial position and velocity vectors in $\mathcal{F}_{I}$. The same initial osculating orbital elements were also used to initialize the proposed 
method of calculating relative position outlined in the previous section. Unless otherwise specified in the captions of the subsequent figures, Table 3.1 presents the initial osculating orbital elements used for both models (numerical simulator and analytical equations). The orbital elements given in this table correspond to a simple in-plane elliptical formation.

Table 3.1: Initial Osculating Orbital Elements

\begin{tabular}{|l|c|c|}
\hline Orbital Element & Leader & Follower \\
\hline Semimajor Axis (km) & 7106.14 & 7106.14 \\
Eccentricity & 0.05 & $e^{\prime}+0.001$ \\
Inclination (deg) & 98.3 & 98.3 \\
Argument of Perigee (deg) & 0 & 0 \\
Right Ascension of Ascending Node (deg) & 270 & 270 \\
True Anomaly (deg) & 0 & 0 \\
\hline
\end{tabular}

Each of the figures in this section presents three analytical solutions for comparison with the numerical simulator. First, the numerical simulator was compared with the original Gurfil and Kholshevnikov equations without any modification, and therefore with no attempt to account for the $J_{2}$ perturbation. Secondly, the numerical simulator is compared to the Gurfil and Kholshevnikov solution using the initial mean orbital elements in the analytical solution instead of initial osculating orbital elements. This represents a case in between using the original Gurfil and Kholshevnikov equations and propagating the $J_{2}$-perturbed osculating orbital elements. It still accounts for the $J_{2}$ perturbation to some extent, namely by evaluating the effect of $J_{2}$ on the initial orbital elements, but does not continue to follow the osculating orbital elements. It is therefore much less computational intensive than the solution proposed in this thesis, but still offers improvement over the original solution. Finally, the numerical simulator is also compared to the proposed equations derived in the previous section. The in-plane motion (on the left side of each figure) and the position error (on the right side of each figure), defined as the difference between the analytical solution and the numerical simulator, are given for each of the three cases, labelled (a), (b) and (c) respectively.

Figure 3.3 reports the results obtained by propagating the relative motion at a relatively low eccentricity over six orbits with the analytical equations and the numerical simulator. The use of the proposed equations greatly reduces error compared 
with the Gurfil and Kholshevnikov solution when the orbit is $J_{2}$-perturbed. This error remains bounded (for all practical purposes) with an accuracy better than 5 meters along each direction. This error is about three orders of magnitude better than the approximately $20 \mathrm{~km}$ spacecraft separation distance. While there is a slight increase in the error in the along-track direction, this minimal drift is negligible over the six orbital periods shown. It is interesting to note that the error when using initial mean orbital elements is significantly improved compared with using initial osculating orbital elements. In other words, a more accurate solution can be obtained from the Gurfil and Kholshevnikov solution by converting the initial conditions to mean orbital elements, which requires little computational effort. If an even more accurate solution is desired, the proposed equations accounting for the short-periodic variations throughout the orbits can be used at the expense of a heavier computational load.

Figures 3.4 and 3.5 show the results obtained when the leader reference orbit is significantly more elliptical. Compared to the previous near-circular orbit case, the errors start to become unbounded, especially in the along-track direction. This is a direct result of the increasing separation distance between the spacecraft in the alongtrack direction as they drift apart with time. It can still be seen that the analytical solution is representative of the exact spacecraft motion. However, it is clear that as the eccentricity increases the ability of the analytical solution to track the relative motion accurately decreases. Once again, this is especially true in the along-track direction, where the error increases significantly with increase in eccentricity. Comparing the proposed equations with both of the Gurfil and Kholshevnikov equations the same trends seen in Fig. 3.3 are observed. Using initial mean orbital elements with the Gurfil and Kholshevnikov solution is a major improvement over using initial osculating orbital elements, but the proposed analytical solution is significantly more accurate than both of these solutions.

Demonstrating that the proposed analytical solution can predict relative postion in highly eccentric orbits is particularly important, as this is where many other formation flying position models fail. To this end, a simulation was performed with $e^{\prime}=0.8$. Figure 3.6 shows that the proposed analytical equations begin to show unbounded error at such high eccentricity values, with large error spikes reaching several 

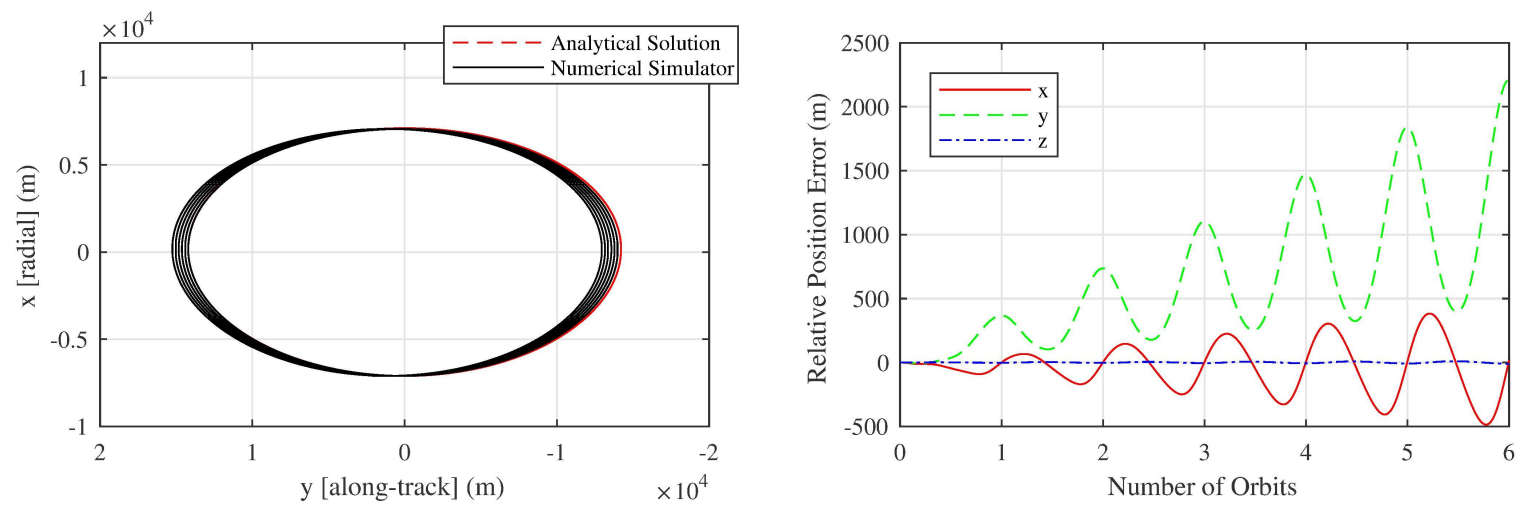

(a) Keplerian Analytical
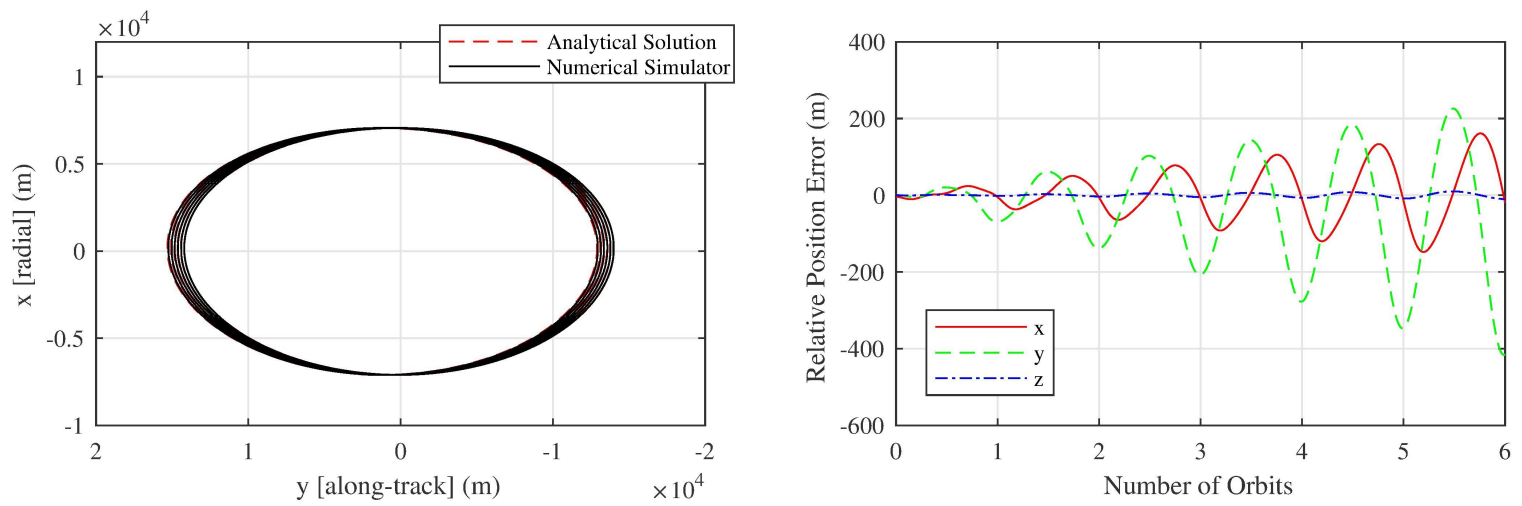

(b) Mean Elements Analytical
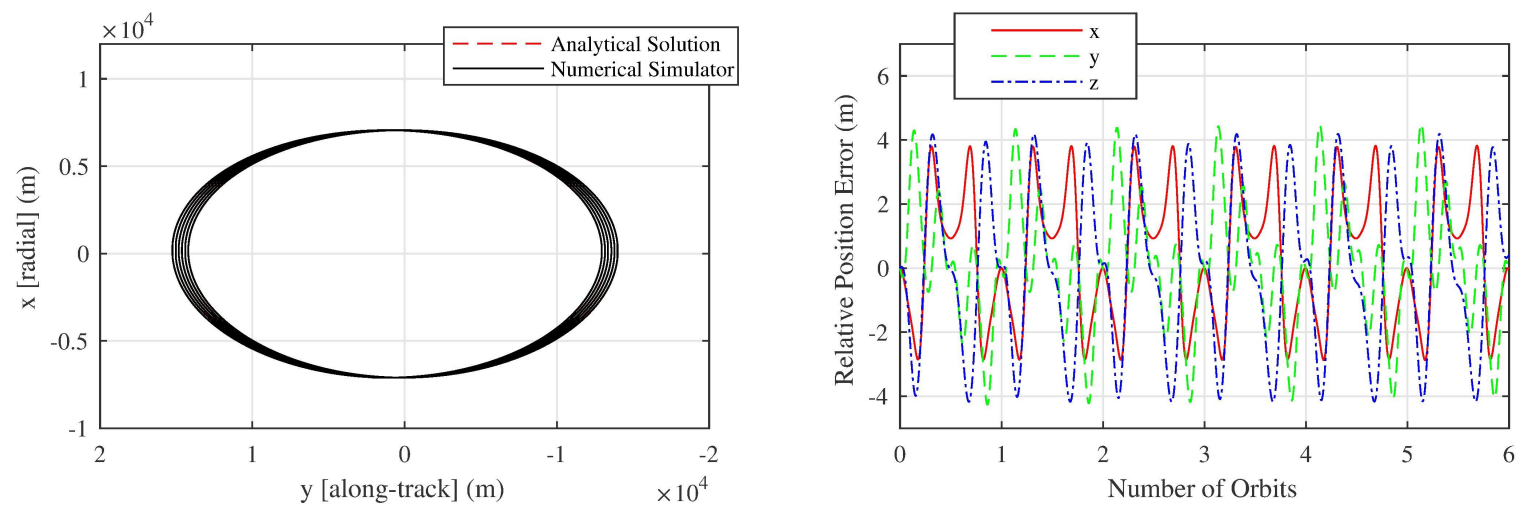

(c) Proposed Analytical Solution

Figure 3.3: Analytical solutions compared with numerical simulator. 

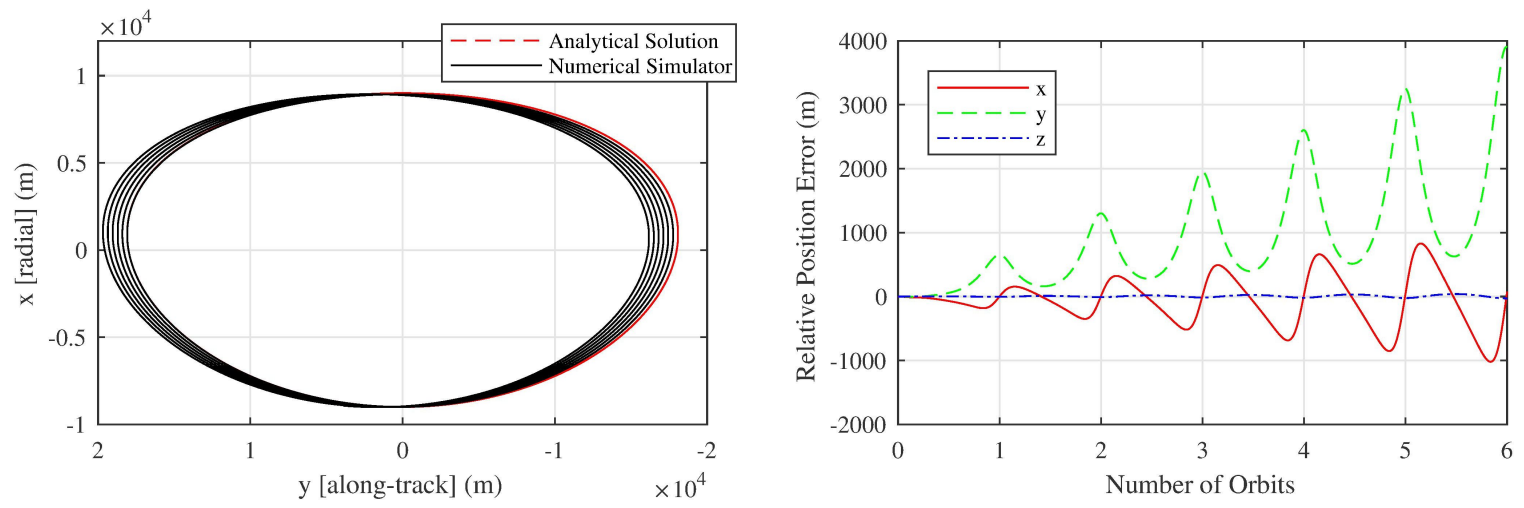

(a) Keplerian Analytical
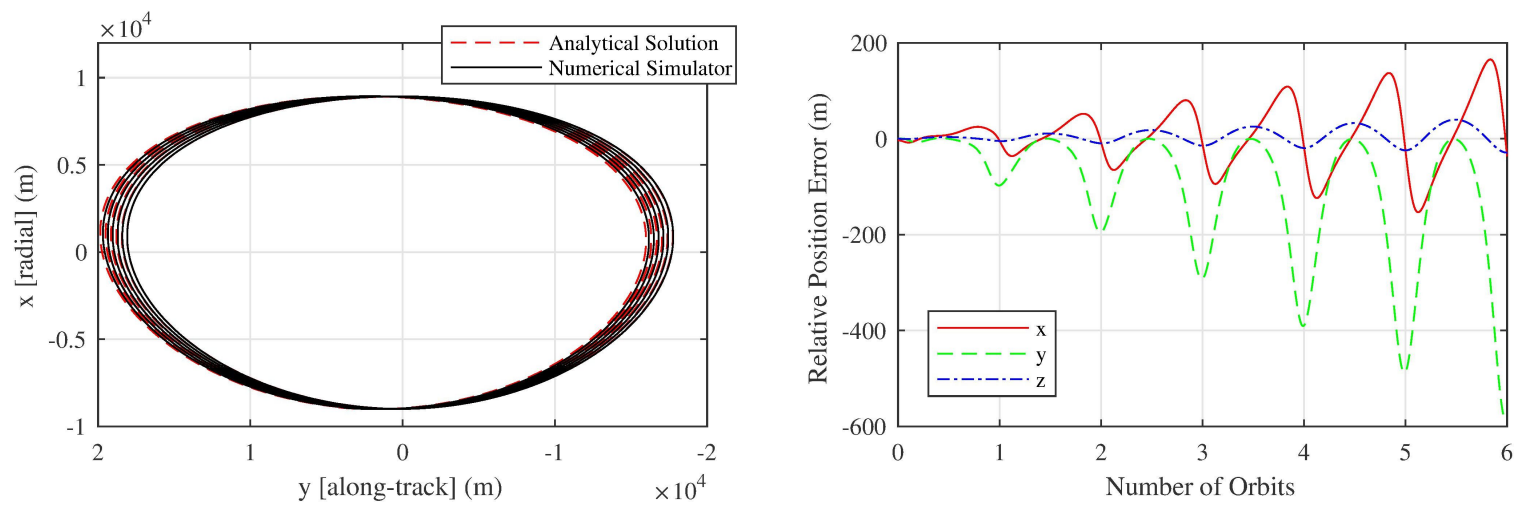

(b) Mean Elements Analytical
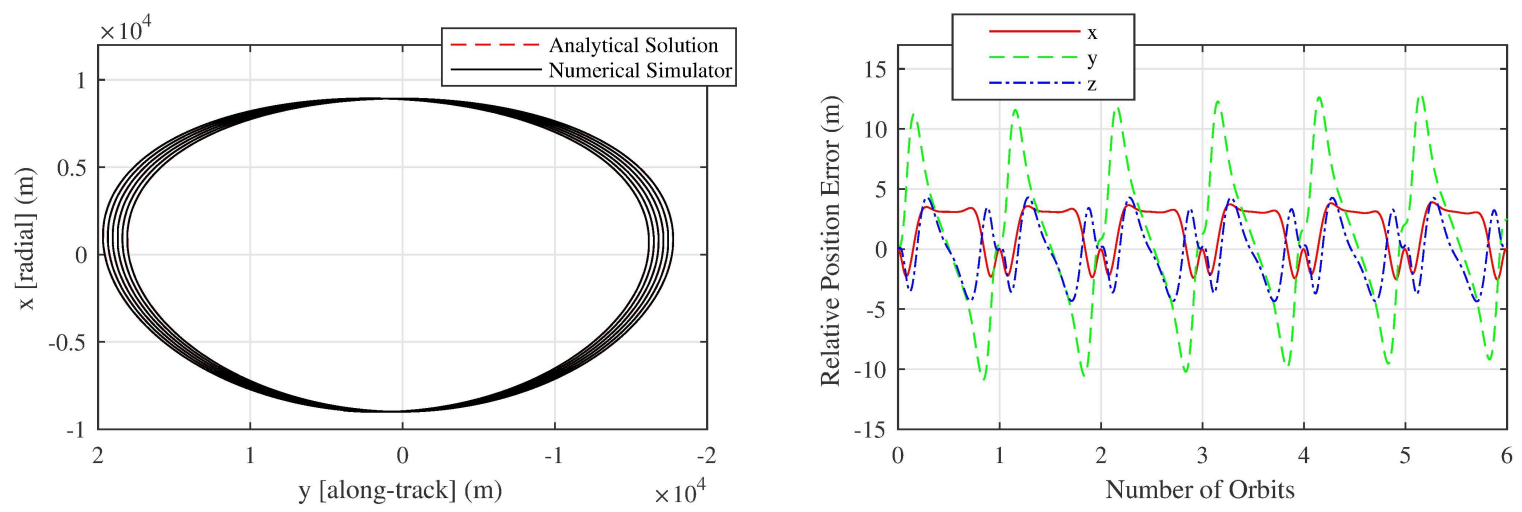

(c) Proposed Analytical Solution

Figure 3.4: Analytical solutions compared with numerical simulator for $e^{\prime}=0.2$ and $a^{\prime}=9,000 \mathrm{~km}$. 

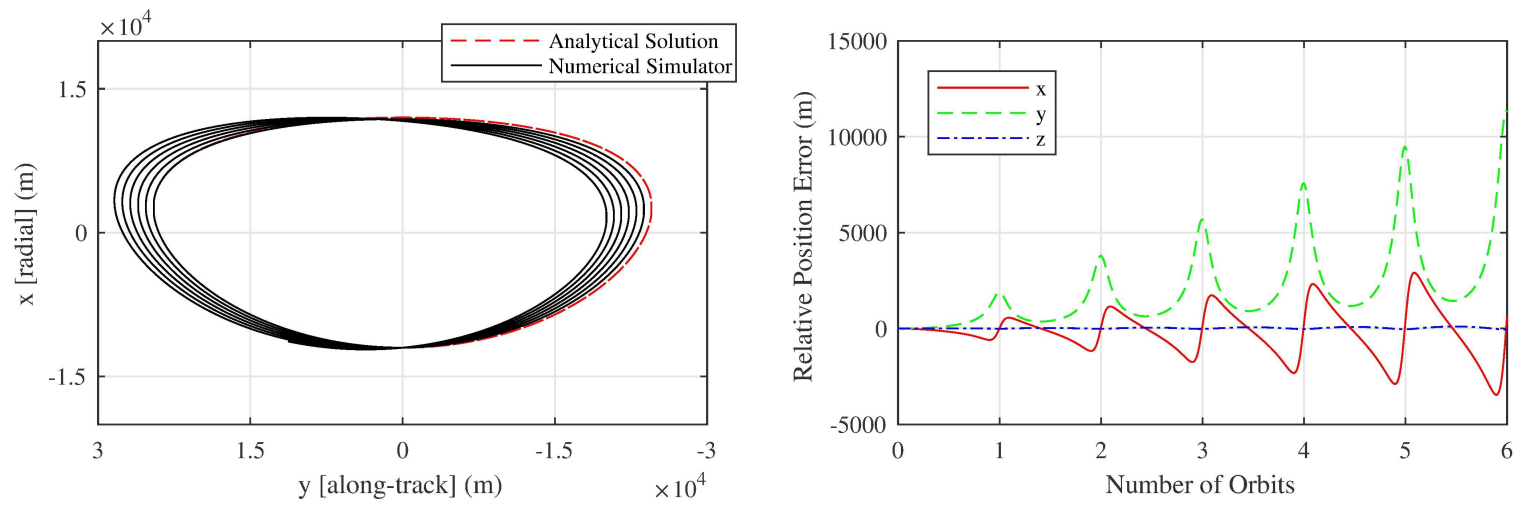

(a) Keplerian Analytical
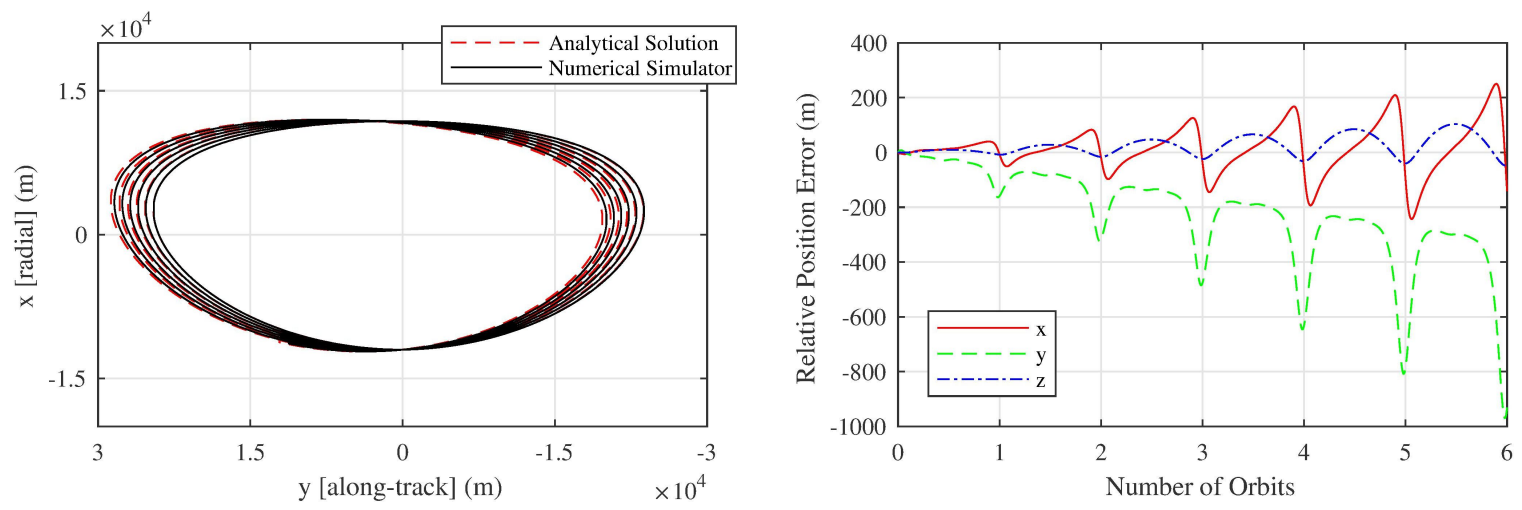

(b) Mean Elements Analytical
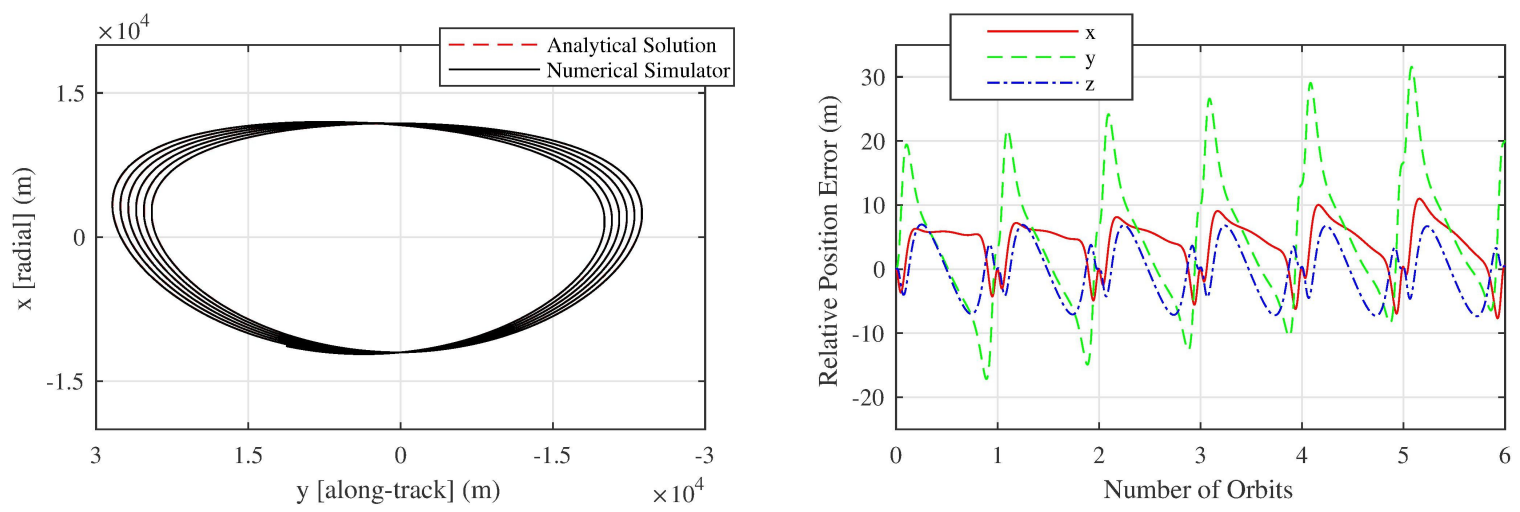

(c) Proposed Analytical Solution

Figure 3.5: Analytical solutions compared with numerical simulator for $e^{\prime}=0.4$ and $a^{\prime}=12,000 \mathrm{~km}$. 
kilometers after six orbits. Looking at the left side of Fig. 3.6 (c) it can be seen that the analytical solution still models the simulated motion despite the increasing error. It can also be seen that over time, as the spacecraft drift apart due to $J_{2}$, the errors get worse. Parts (a) and (b) of the figure, as expected, do continue to show that the proposed equations are still a significant improvement over the Gurfil and Kholshevnikov solution. It should be noted that the accuracy of Eq. (3.23) is a function of the number of iterations performed in the equation. When the eccentricity was increased to 0.8, the resulting errors were initially significantly larger. By increasing the iterations in Eq. (3.23) this error was reduced to the results shown in Fig. 3.6.

To further study the application of the proposed analytical solution for formations on highly-eccentric orbits, the relative equations of motion were applied to the European Space Agency's PROBA-3 mission. This Sun observation mission involves two spacecraft in precise formation in highly-eccentric orbits, with the formation forming a solar coronagraph. The PROBA-3 leader's orbit is defined with $a_{0}^{\prime}=37040 \mathrm{~km}$, $e_{0}^{\prime}=0.806, i_{0}^{\prime}=59 \mathrm{deg}, \Omega_{0}^{\prime}=84 \mathrm{deg}$, and $\omega_{0}^{\prime}=188 \mathrm{deg}$, as well as a spacecraft separation on the order of a few hundred meters.[29] Setting up a simulation with two spacecraft in this orbit, with the follower eccentricity given by $e=e^{\prime}+0.000005$ is a gross simplification of the ultimate relative motion for the mission, but it may still provide some insight regarding the accuracy of the proposed analytical solution in a realistic mission scenario. Figure 3.7 shows the results of the simulation. Although the resulting errors are unbounded, the error is kept relatively low using the proposed analytical equations, staying below 30 meters over six orbital periods. Although this might seem to be a large amount of error, it should be stressed that after the six orbits the spacecraft are now separated by about 2 kilometers when the error spike of approximately 30 meters occurs, indicating that the the accuracy is still about two orders of magnitude of the separation distance.

In each of the previous cases, the formation was defined such that there is no out-of-plane motion. As it is important to assess the ability of the analytical solution to handle out-of-plane motion effectively, three more cases were simulated at varying inclination differences between the two spacecraft. The results, in order of increasing inclination difference are reported in Figs. 3.8, 3.9, and 3.10. Each of these figures 

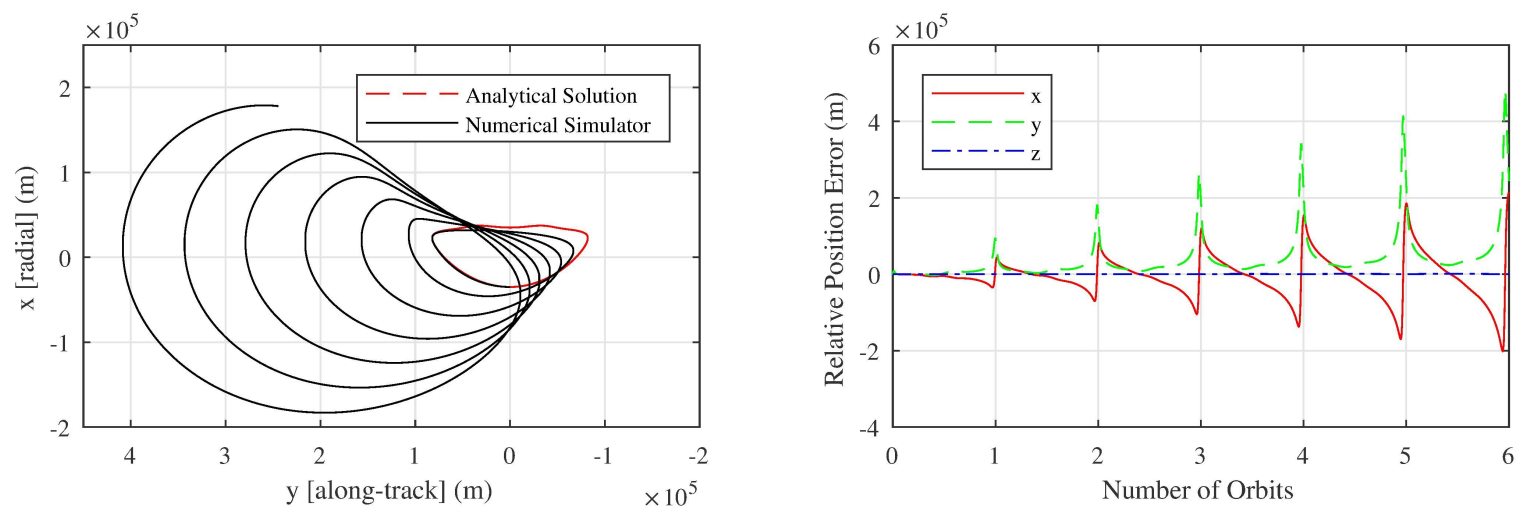

(a) Keplerian Analytical
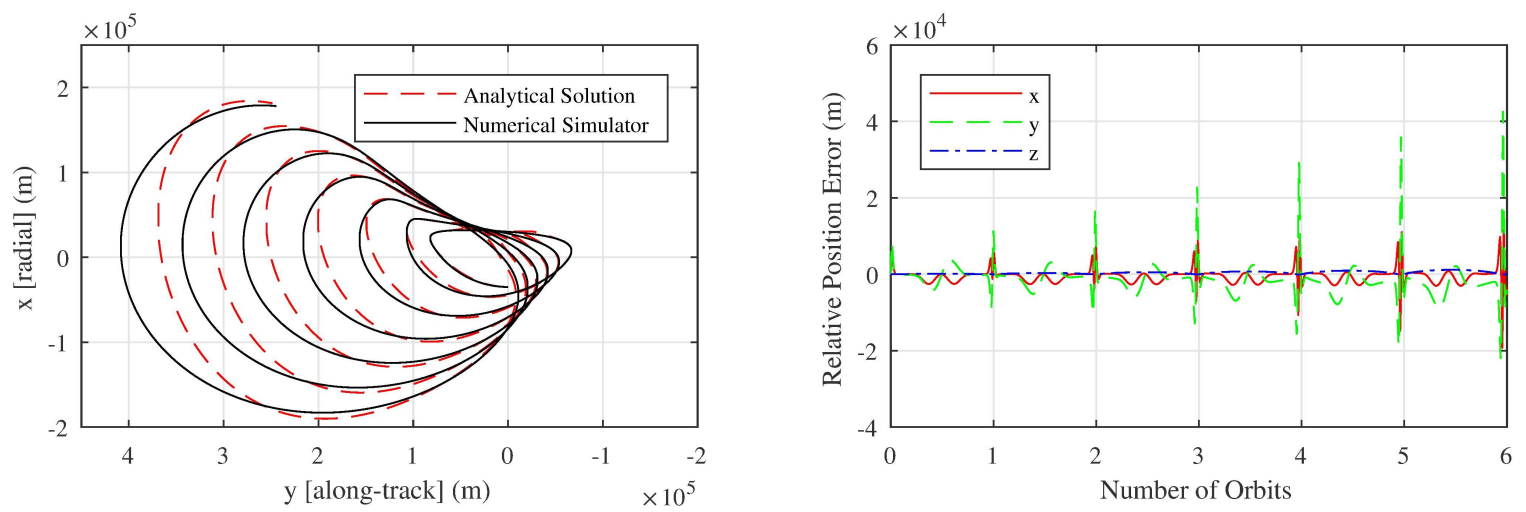

(b) Mean Elements Analytical
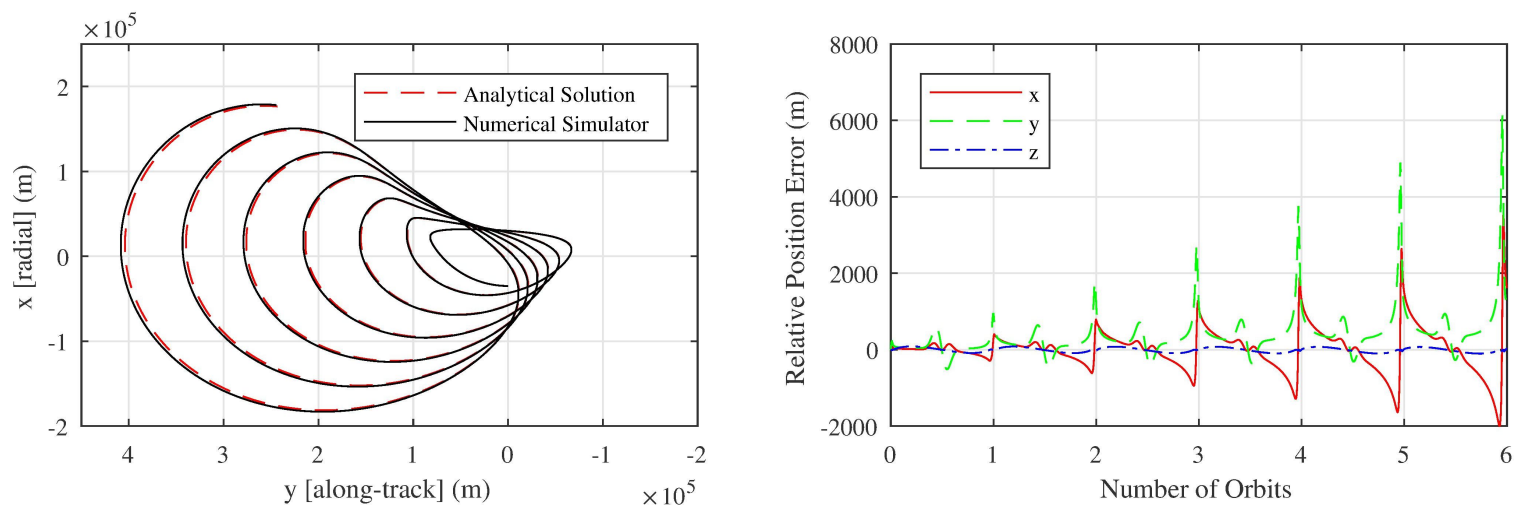

(c) Proposed Analytical Solution

Figure 3.6: Analytical solutions compared with numerical simulator for $e^{\prime}=0.8$ and $a^{\prime}=35,000 \mathrm{~km}$. 

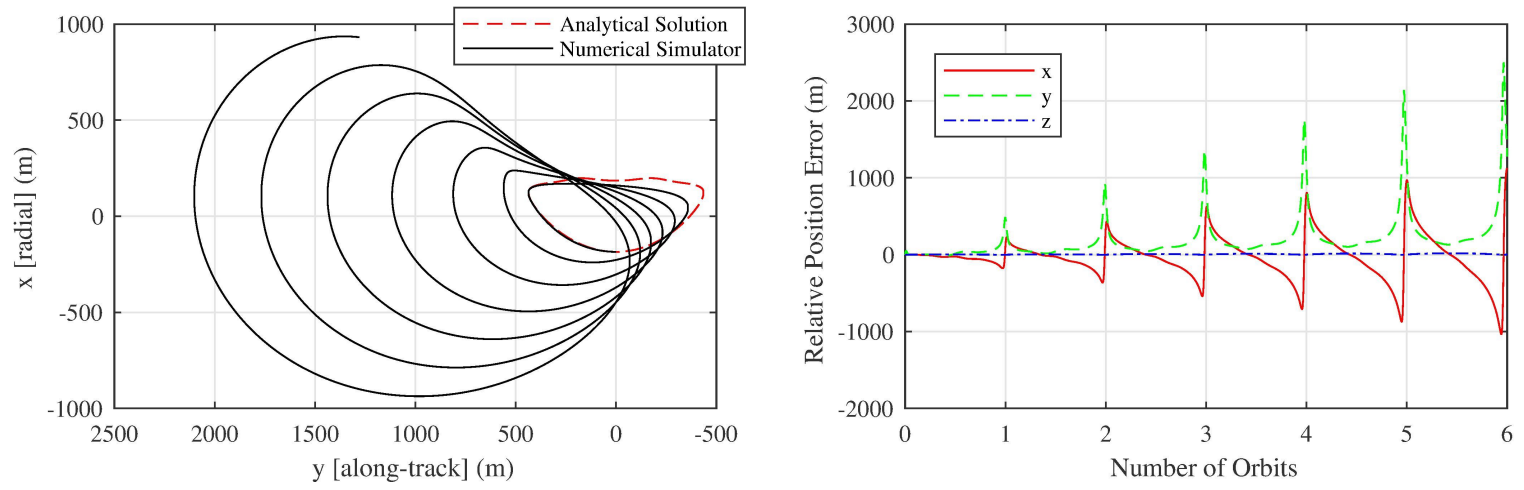

(a) Keplerian Analytical
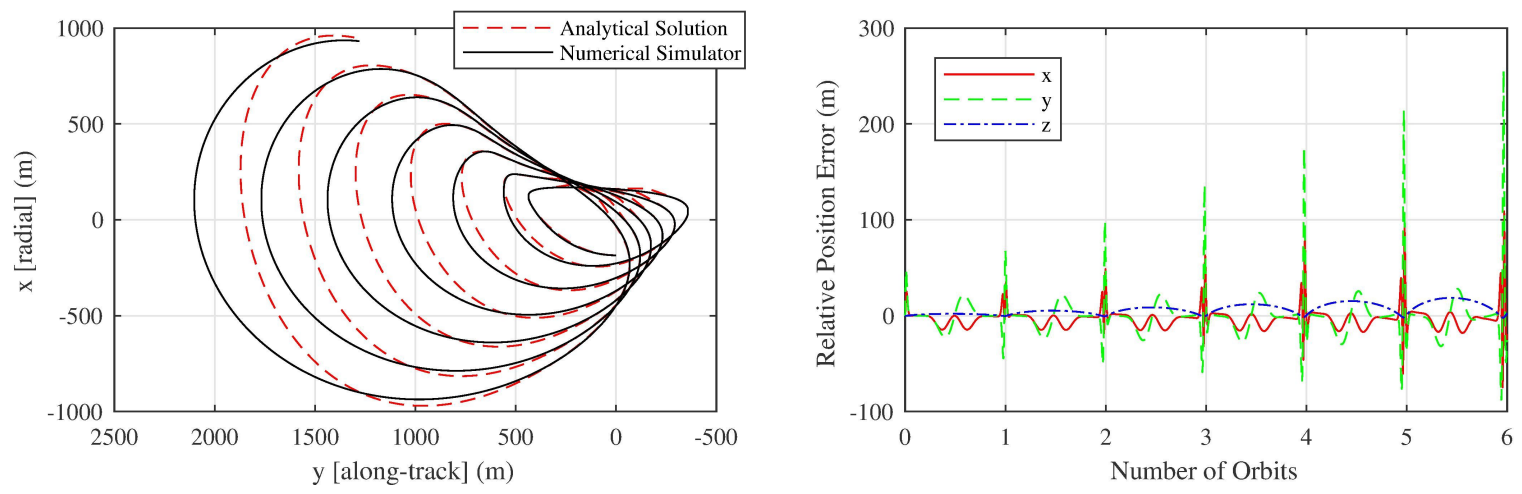

(b) Mean Elements Analytical
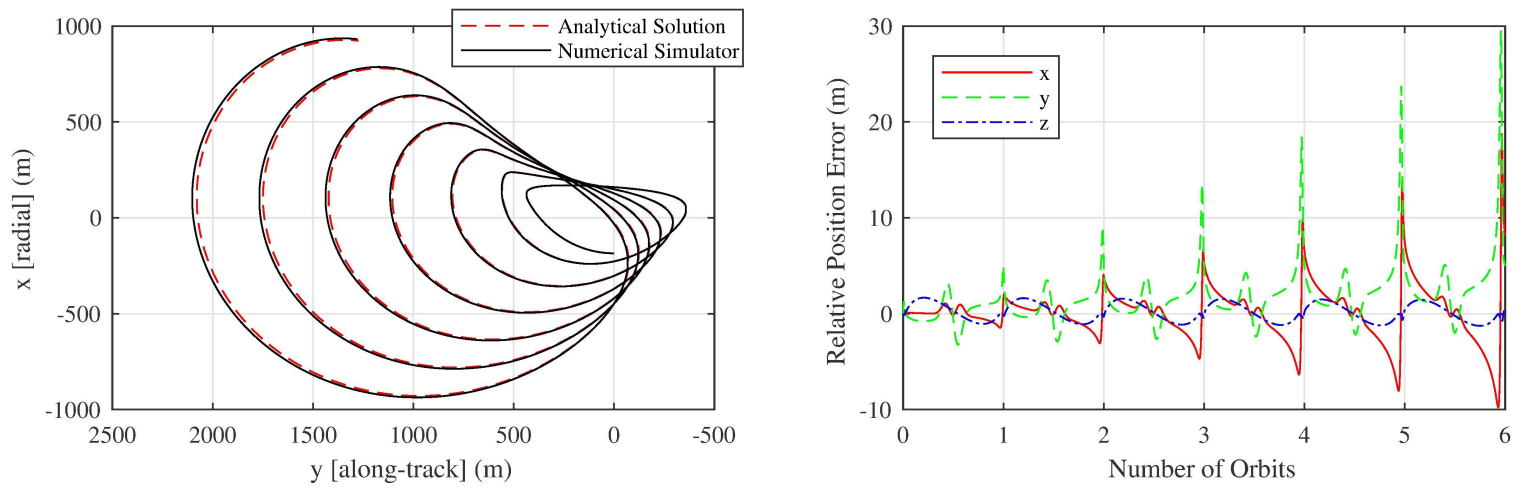

(c) Proposed Analytical Solution

Figure 3.7: Analytical solutions compared with numerical simulator for PROBA-3 example. 
demonstrates that the proposed analytical solution is once again an improvement over the Gurfil and Kholshevnikov solutions, as well as validating that the proposed solution can efficiently handle out-of-plane motion as well as in-plane motion. In these cases the out-of-plane accuracy is within approximately three orders of magnitude of the out-of-plane separation distance.

Another interesting note that should be made is that the error differences between using initial mean and initial osculating orbital elements is greatest if the initial conditions are near perigee. The reason for this is that the short-periodic variations affecting most of the orbital elements tend to reach their highest magnitudes near these points. Intuitively, at orbit points where the short-periodic variations are smallest, the difference between mean and $J_{2}$-perturbed osculating orbital elements is small, and the errors resulting from initializing the Gurfil and Kholshevnikov solution with either of them become similar. Figure 3.11 demonstrates this by showing error results for the three different cases when the orbits are initialized at perigee and apogee.

The far right case shows the results from using the proposed equations defined in this thesis. The other two cases both use the Gurfil and Kholshevnikov equations, but the plot in the middle uses initial mean orbital elements while the plot on the right uses initial osculating orbital elements to initialize the propagation of the motion. To get the initial mean orbital elements, the initial short periodic variations were substracted from the defined initial orbital elements (note that this plot used the same initial orbital elements as Fig. 3.4).

Finally, it is important to discuss the dependence of the errors on a few additional factors. The first is that, intuitively, the magnitude of the error is directly correlated with the separation distance between the spacecraft. In most of the previous examples relatively large separation distances were used, typically on the order of 10 to 20 kilometers. Reducing that separation distance to a few hundred meters reduces the resulting error between the proposed equations and numerical simulator to well under a meter in many cases, although the trends tend to stay the same. Fig. 3.12 shows the error resulting from using the same initial conditions as Fig. 3.4, but with an eccentricity difference between leader and follower of 0.00001 instead of 0.001 . 

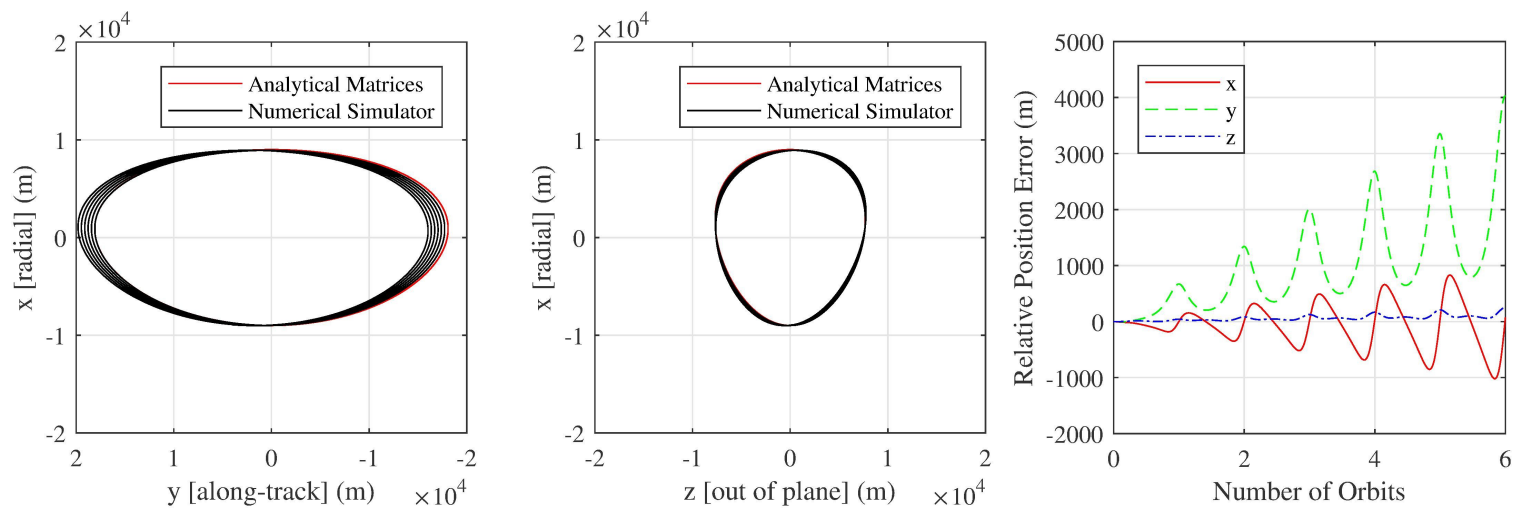

(a) Keplerian Analytical
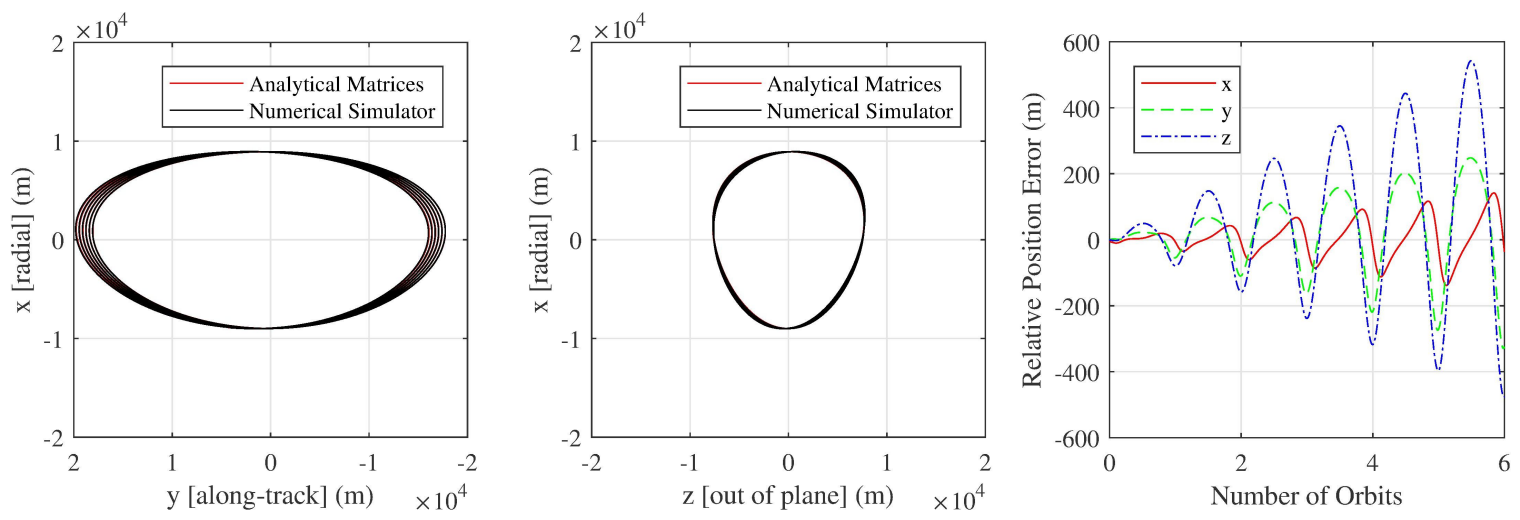

(b) Mean Elements Analytical
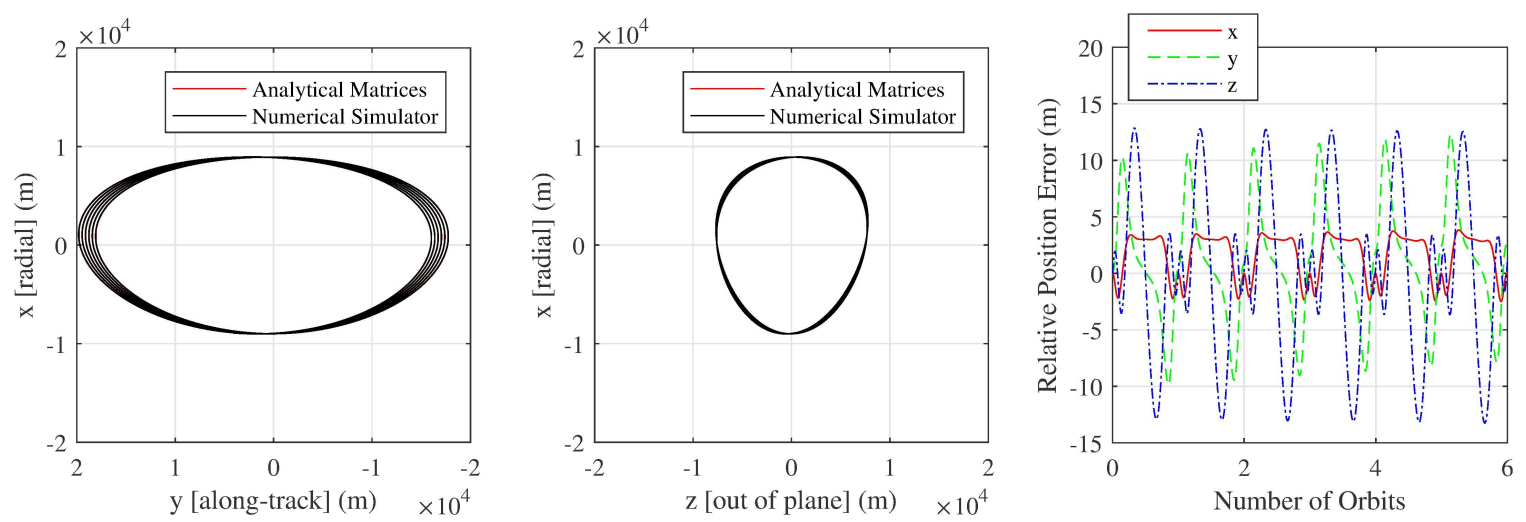

(c) Proposed Analytical Solution

Figure 3.8: Analytical solutions compared with numerical simulator for $e^{\prime}=0.2$ and $a^{\prime}=9,000 \mathrm{~km}$ and $i=i^{\prime}+0.05 \mathrm{deg}$. 

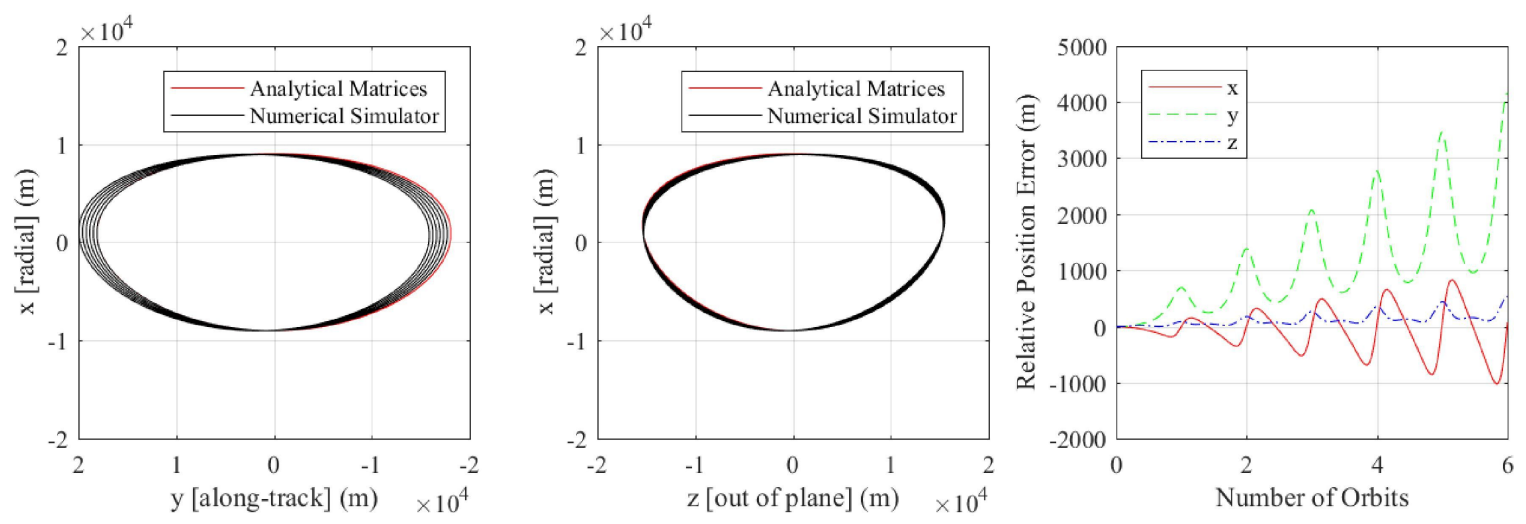

(a) Keplerian Analytical
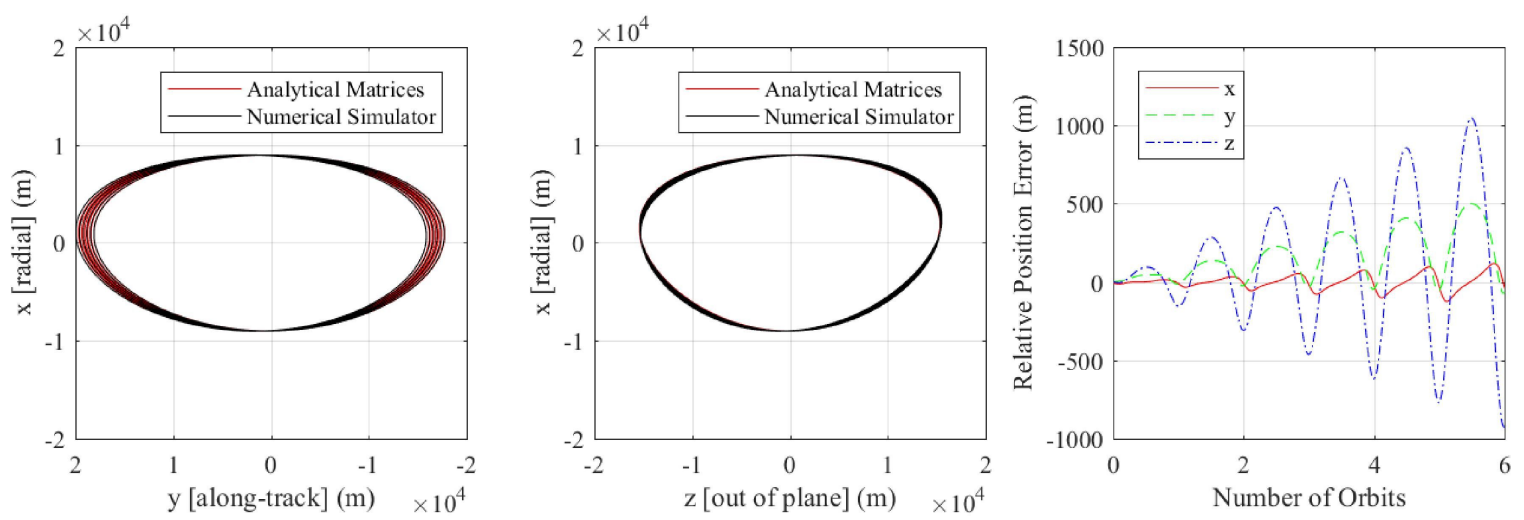

(b) Mean Elements Analytical
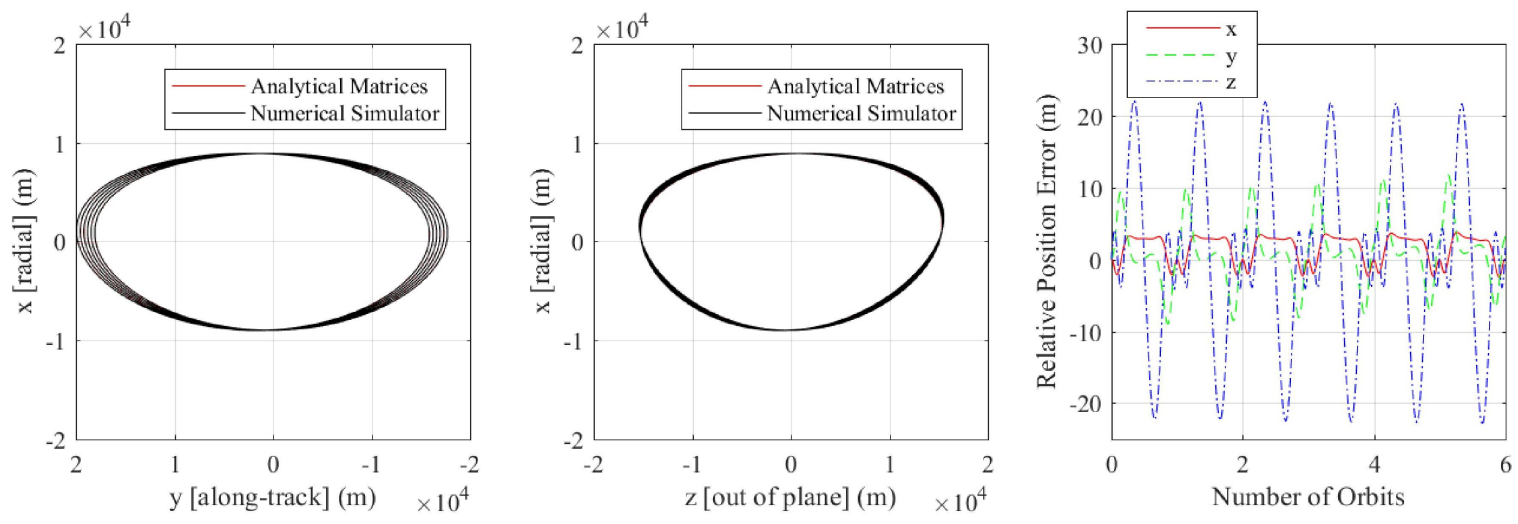

(c) Proposed Analytical Solution

Figure 3.9: Analytical solutions compared with numerical simulator for $e^{\prime}=0.2$ and $a^{\prime}=9,000 \mathrm{~km}$ and $i=i^{\prime}+0.1 \mathrm{deg}$. 

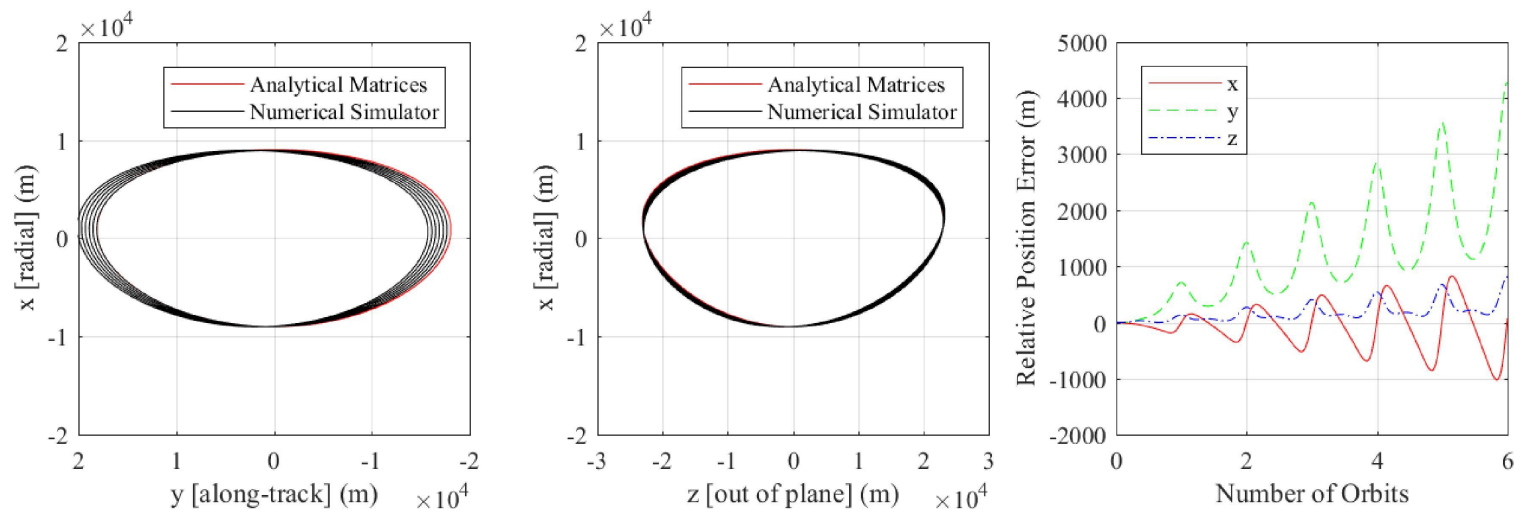

(a) Keplerian Analytical
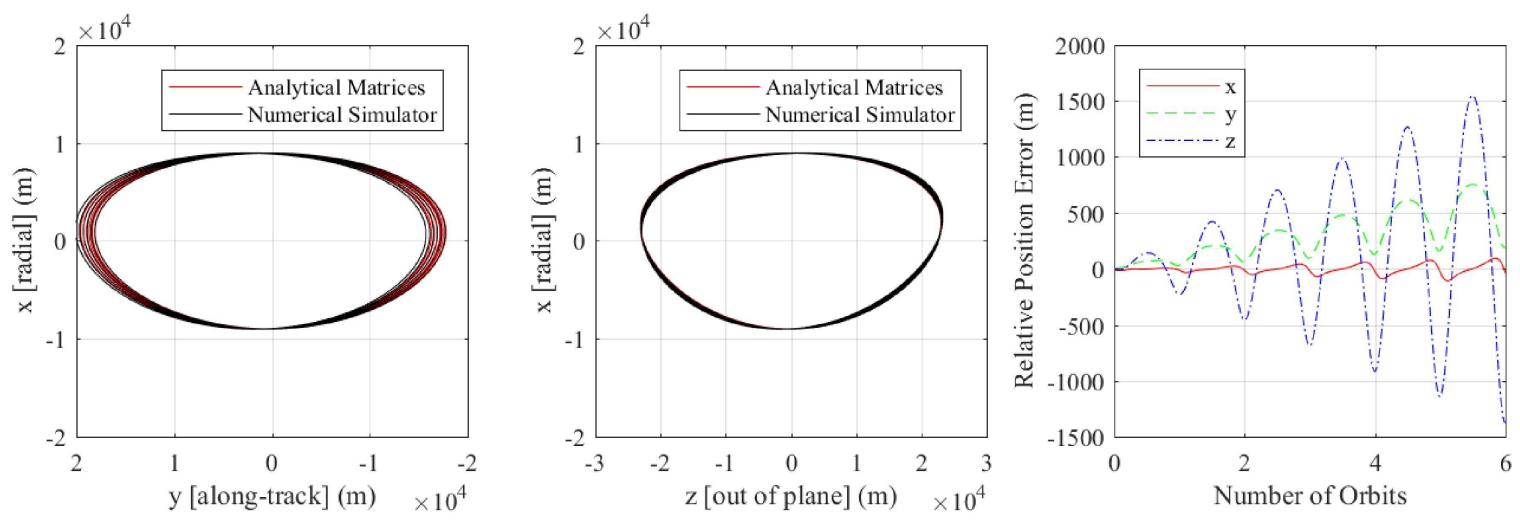

(b) Mean Elements Analytical
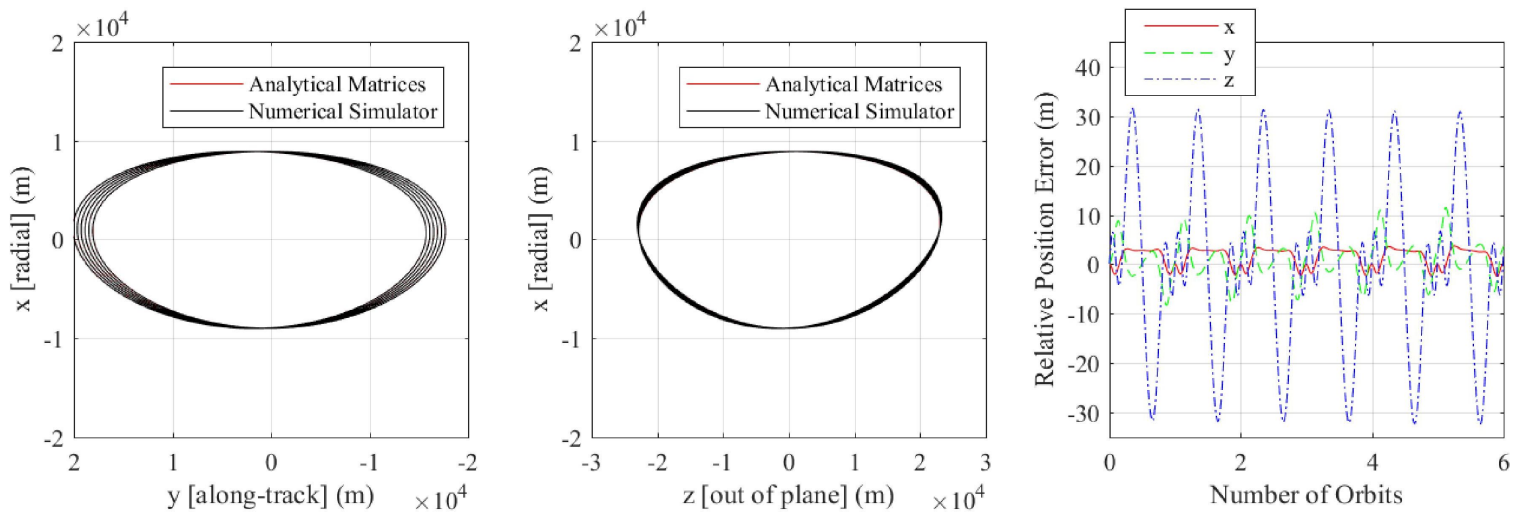

(c) Proposed Analytical Solution

Figure 3.10: Analytical solutions compared with numerical simulator for $e^{\prime}=0.2$ and $a^{\prime}=9,000 \mathrm{~km}$ and $i=i^{\prime}+0.15 \mathrm{deg}$. 

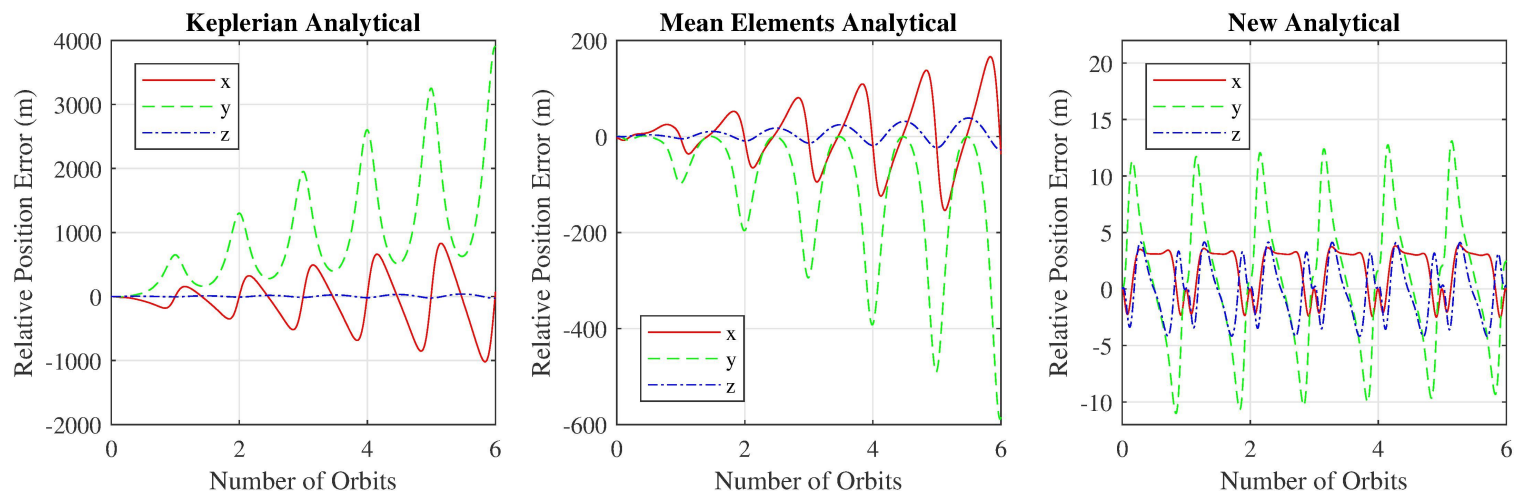

(a) Initial true anomaly of 0 degrees
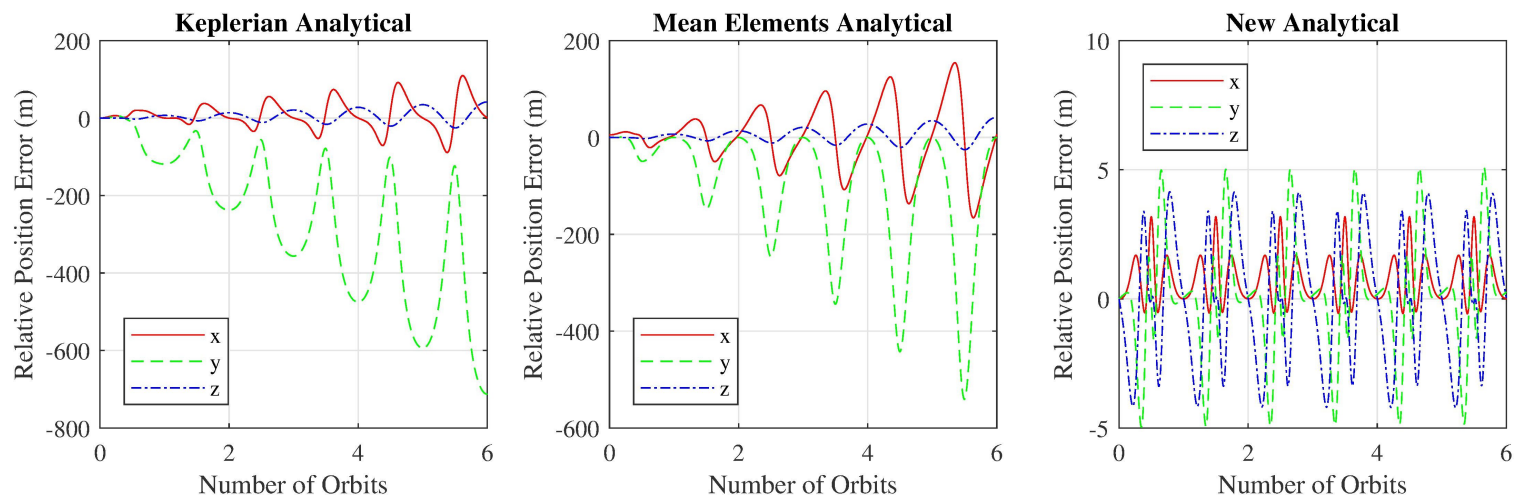

(b) Initial true anomaly of 180 degrees

Figure 3.11: Comparison of error resulting from the Proposed equations of motion and the Gurfil and Kholshevnikov solution with different initial true anomaly values.
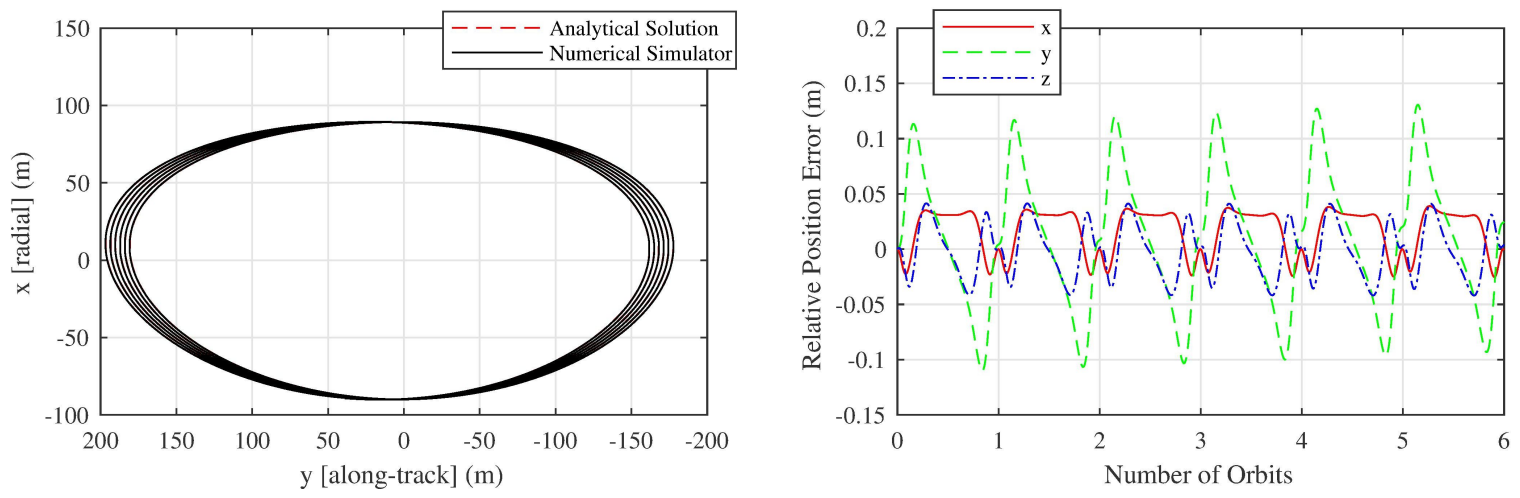

Figure 3.12: Analytical solution compared with numerical simulator for $e^{\prime}=0.2$ and $a^{\prime}=9,000 \mathrm{~km}$ and $\mathrm{e}=0.20001$. 
Looking through the figures in this section it can be seen that the error in each direction often sees spikes in magnitude at particular times. Often, these spikes appear to show up near the perigee of the orbits of the spacecraft in formation. This interesting result may be evidence that the short-periodic variations in the orbital elements are less accurate at this point, or perhaps that other variations in the $J_{2}$-perturbed osculating orbital elements which are not accounted for (long-periodic variations) peak at perigee. Looking at Figs. 3.13 and 3.14, it is clear that peaks in short-periodic variation magnitude tend to occur near perigee. Perhaps a more interesting result is the fact that the peak short-periodic variation in each orbital element is much larger in the higher eccentricity case, especially near perigee. This gives some insight into why the proposed equations, although they manage to follow the trend of the simulated motion, show larger errors at higher eccentricity values. 

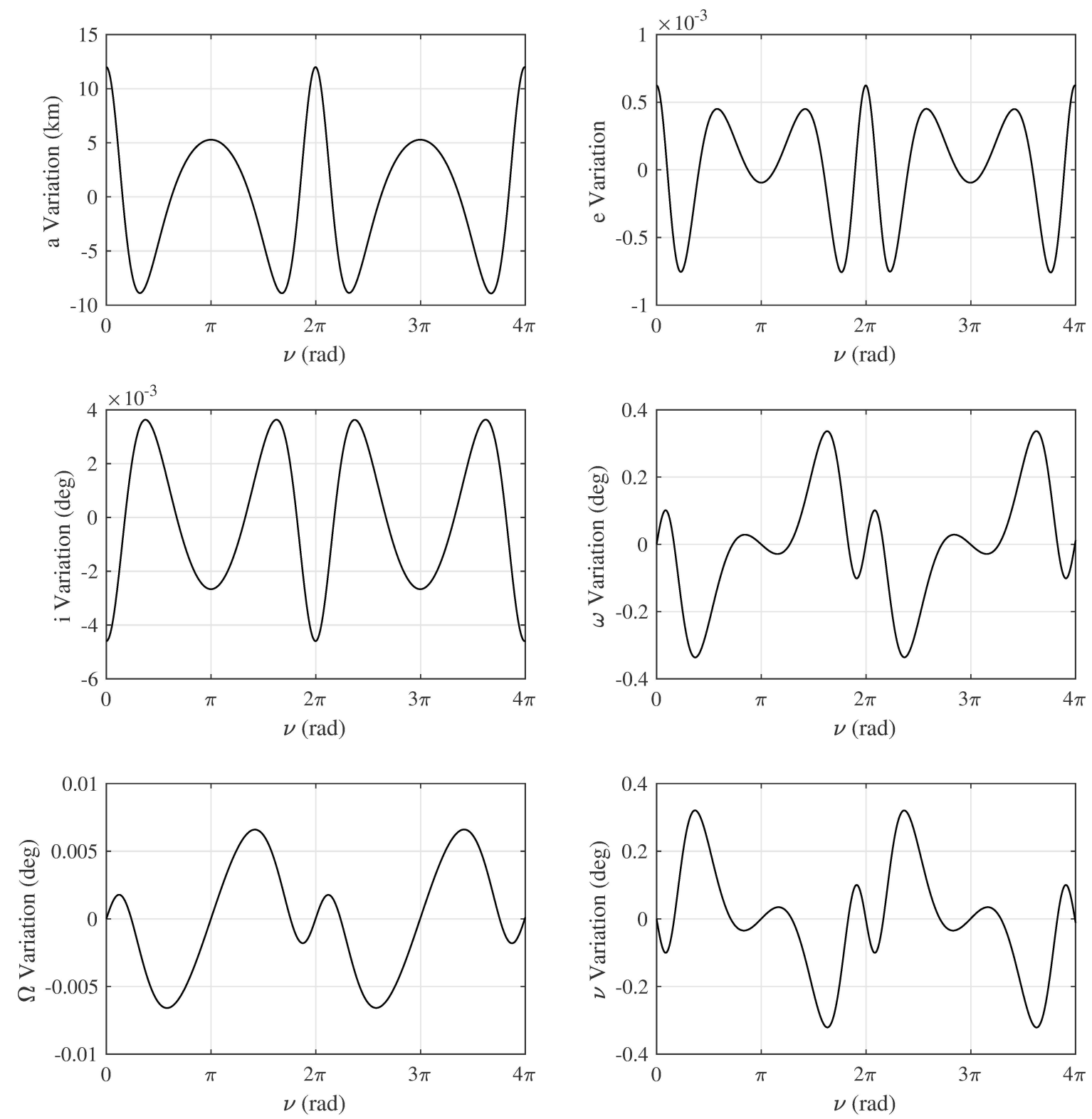

Figure 3.13: Short-periodic variation of each orbital element with time plotted with true anomaly for the $e^{\prime}=0.2$ and $a^{\prime}=9,000 \mathrm{~km}$ case. 

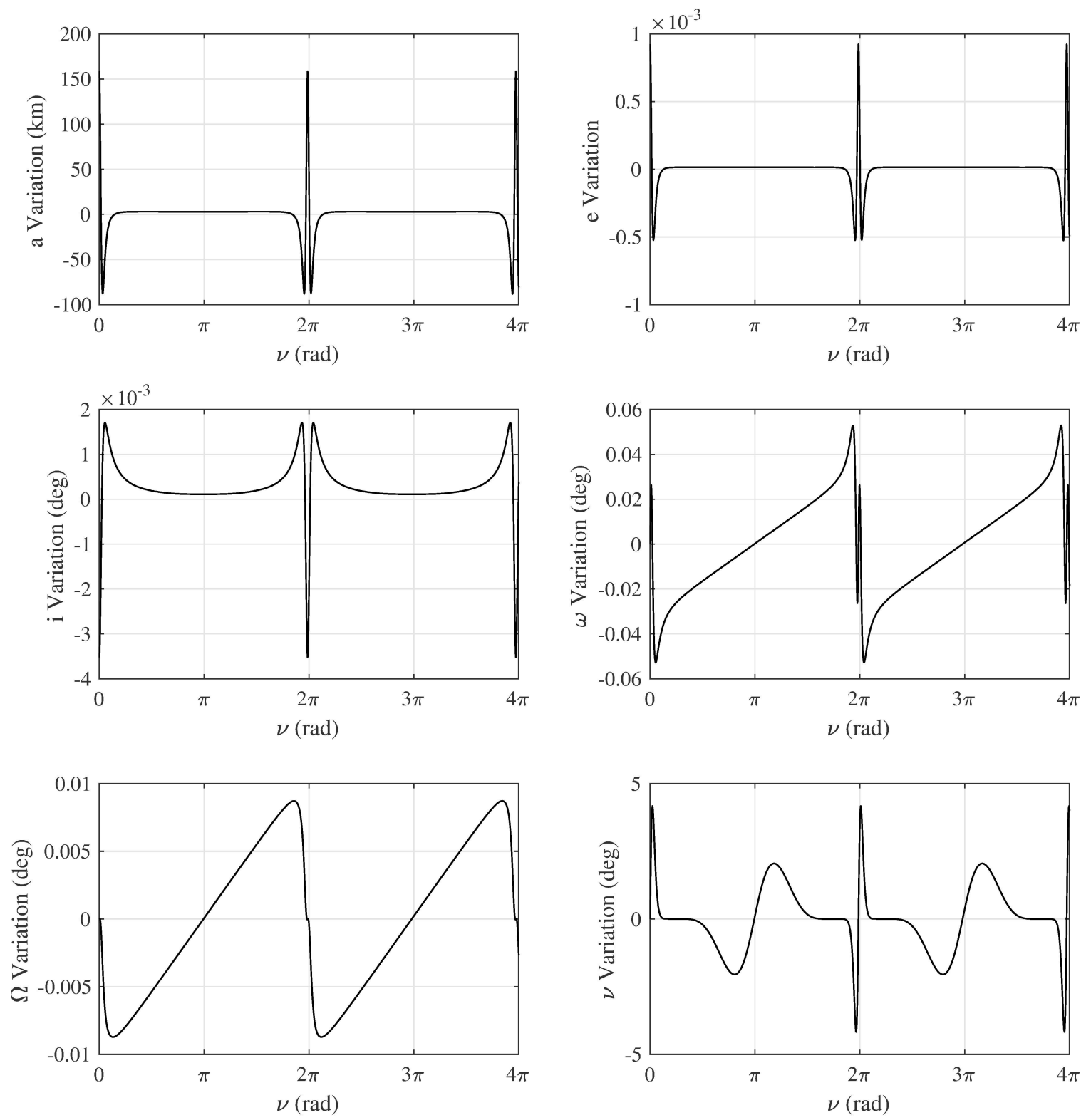

Figure 3.14: Short-periodic variation of each orbital element with time plotted with true anomaly for the $e^{\prime}=0.8$ and $a^{\prime}=35,000 \mathrm{~km}$ case. 


\section{Chapter 4}

\section{Terminal Point Guidance}

This Chapter presents the first use of the newly-developed equations of relative motion in a practical application. This involves the novel concept of back-propagation, which will be defined in this chapter and which is used in combination with the proposed equations in an effort to perform more efficient reconfiguration maneuvers. The back-propagation method is combined with an impulsive controller to simulate a reconfiguration maneuver.

\subsection{Introduction}

Another advantage to the proposed method of determining relative motion of spacecraft in formation is that the equations can be propagated both backward and forward in time. This has potential applications in spacecraft formation flying, as often-times the desired final motion of the spacecraft is known, but at the present the spacecraft may be tracking some unrelated motion. By taking the desired spacecraft dynamics at some known time in the future, and back-propagating through the previously presented equations of relative motion, the ideal motion of each spacecraft at the present time can be calculated. That is, the position and velocity, or corresponding set of orbital elements, of each spacecraft at the present time which will result in the desired formation at the future time can be calculated. In many situations this should result in fuel savings, as instead of letting the spacecraft track some arbitrary relative motion until a specific relative motion is required and then performing the required maneuvers, the spacecraft are initially placed (using smaller maneuvers) onto a trajectory that naturally drifts, due to $J_{2}$, towards the desired final formation. In this section the magnitude of the maneuvers will be compared in terms of $\Delta v$, the change in spacecraft velocity required to place the spacecraft in the desired orbit.

Before implementing the proposed method of back-propagation for more efficient 
spacecraft maneuvers with the proposed equations of relative motion, a simple proof of concept was set up using Hill's, or the Clohessy-Wiltshire, equations (HCW model) [6]. This set of three equations gives the position of a follower spacecraft relative to the leader as function of time based on the initial relative position and velocity. The HCW equations were shown in Sec. 2.8.

Section 4.2: Back-Propagation of Proposed Equations validates the back-propagation concept with the proposed analytical equations of formation motion, and also describes an impulsive controller with will be paired with the proposed equations in a closed-loop guidance and control simulation. Section 4.3 presents the results of the closed-loop implementation.

\subsection{Back-Propagation of Proposed Equations}

Back-propagation can be implemented through the new nonlinear $J_{2}$-perturbed analytical equations of relative motion in a similar manner to what is commonly done for the HCW linear equations of motion. Instead of starting a scenario with initial conditions, the scenario is started with desired final conditions in the form of final osculating orbital elements for each spacecraft. These elements are then propagated backward from zero to the negative of the final time, while taking into account the $J_{2}$ perturbation. Finally, along the way, the relative position of the two spacecraft can be solved for, based on the time-varying orbital elements.

A simulation was performed to validate the accuracy of the back-propagation guidance strategy. A desired final relative position of spacecraft was defined, and then propagated backward to the initial time. The relative position at the initial time was then propagated forward in time with a numerical simulator. Figure 4.1 compares the time-history of the relative position components in $\mathcal{F}_{\mathcal{L}}$ obtained from the back-propagation guidance strategy against those obtained from the numerical simulator.

As can be seen in Fig. 4.1, there are no noticeable differences between the numerical simulator and the proposed analytical solution. Figure 4.2 takes a closer look at the differences by comparing the time-history of the error of the relative position components in $\mathcal{F}_{\mathcal{L}}$ obtained from the back-propagation guidance strategy compared 

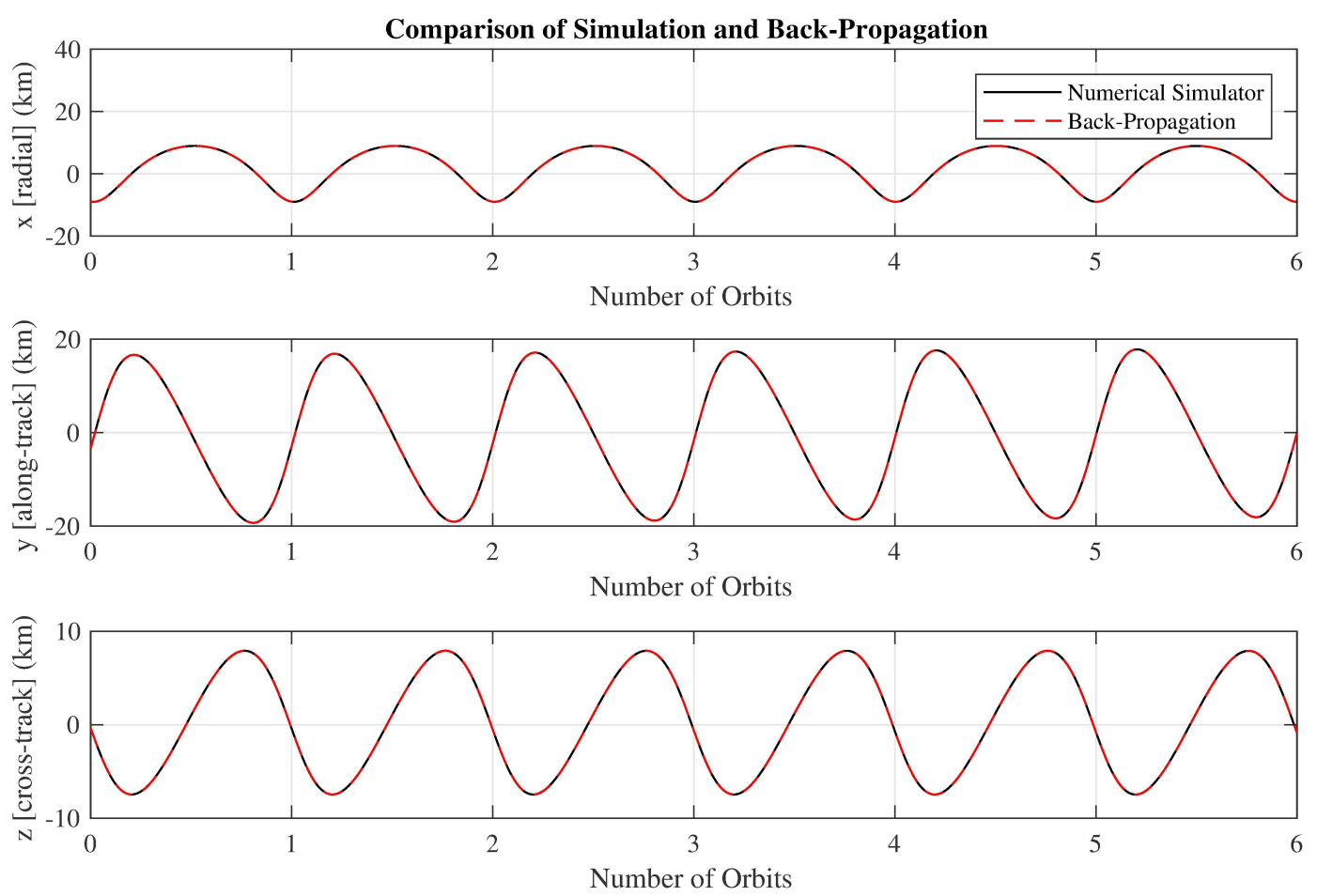

Figure 4.1: Comparison of relative position from numerical simulator and backpropagation (note that the formation is the same as defined in Fig. 3.8)

against those obtained from the numerical simulator.

Looking at Fig. 4.2, it can be seen that, although there are small differences between the relative positions obtained from back-propagation and the numerical simulation, the back-propagation works well, with errors on the order of 5 to 10 meters as would be expected based on the results presented in Sec. 3.3. These results demonstrate that the concept of back-propagation guidance can be used with the proposed equations to accurately determine the necessary initial conditions of spacecraft in formation to passively achieve some desired final relative position in the future.

\subsubsection{Closed-Loop Implementation}

One potential application of the back-propagation guidance strategy is to perform more efficient reconfiguration maneuvers. Instead of performing maneuvers at the time that the spacecraft are required to be in a specific configuration, more efficient maneuvers can be performed at some earlier time, placing the spacecraft in a position which allows them to drift naturally into the desired final configuration. 

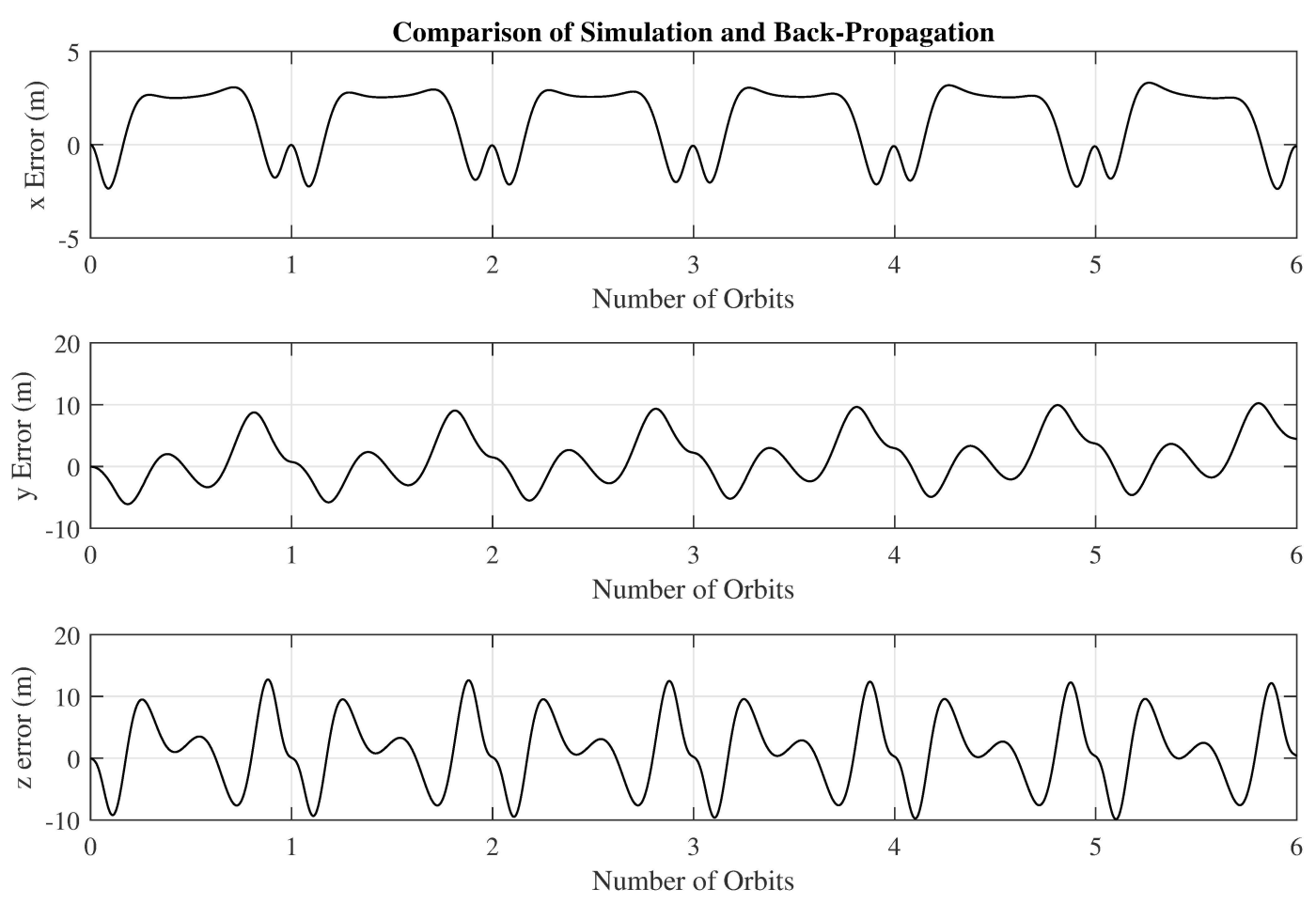

Figure 4.2: Relative position error of back-propagation compared with numerical simulator (note that the formation is the same as defined in Fig. 3.8)

To test the feasibility of this concept, let us assume an active follower spacecraft to be maneuvered closer to an uncontrolled leader spacecraft. The orbital elements of the leader are known, as is a desired set of orbital elements for the follower spacecraft corresponding to a simple in-plane elliptical formation, which should be achieved within ten orbital periods of the leader. The orbital elements for the initial position of the follower are also known.

Two cases can be compared in this scenario, with both accomplishing the desired reconfiguration maneuvers after ten orbital periods. In the first case, a maneuver is performed to achieve the desired final formation such that it will be completed just prior to the time that the formation is required, based on the motion of each spacecraft at that time. In the second case, a more efficient strategy is implemented, which corresponds to the newly-developed back-propagation guidance law that finds the set of orbital elements at the initial time which will lead to the desired final orbital elements at the final time. A set of maneuvers is applied as soon as possible to achieve this ideal set of initial orbital elements, and the spacecraft is allowed to 
drift naturally into the desired configuration, thereby minimizing fuel consumption.

\subsubsection{Control Method Selection}

To assess the potential benefits of the proposed back-propagation method in a closedloop scenario, the back-progagation guidance law is combined with the impulsive feedback control method proposed by Schaub and Alfriend [30]. This particular control scheme is convenient, as the impulsive maneuvers are quite simple to implement, and the $\Delta v$ maneuvers are defined in terms of orbital element differences. For completeness, this impulsive control law is summarized in this subsection.

This control method consists of five different impulsive maneuvers which are applied at three different locations over the course of an orbit. The first maneuver corrects the inclination and the right ascension of ascending node of the spacecraft, and is fired in the orbit normal direction, the magnitude of the impulse is given by

$$
\Delta v_{h}=(h / r) \sqrt{\delta i^{2}+\delta \Omega^{2} \sin \left(i^{2}\right)}
$$

where $h$ is orbital angular momentum, $r$ is the spacecraft position magnitude, and $\delta i$ and $\delta \Omega$ are the orbital element differences, defined as the desired final orbital element subtracted by the current orbital element. This maneuver must take place at a critical argument of latitude angle defined as

$$
u_{c}=\tan ^{-1}(\delta \Omega \sin (i) / \delta i)
$$

This first maneuver is only meant to affect the inclination and right ascension of ascending node, however it should be noted that it will also affect the argument of perigee. This effect will be accounted for in the equations for the next maneuvers, which correct the argument of perigee and mean anomaly errors. To correct these

two elements, two maneuvers are performed, one at perigee and one at apogee, each in the radial direction. The two maneuvers are defined in the following equations

$$
\Delta v_{r_{p}}=-(n a / 4)\left\{\left[(1+e)^{2} / n\right](\delta \omega+\delta \Omega \cos (i))+\delta M\right\}
$$




$$
\Delta v_{r_{a}}=(n a / 4)\left\{\left[(1-e)^{2} / n\right](\delta \omega+\delta \Omega \cos (i))+\delta M\right\}
$$

The final two manuevers are performed to correct the semi-major axis and the eccentricty error, with one taking place at perigee and one at apogee. In this case, each maneuver is fired in the tangential direction, and the magnitude of the respective impulsive maneuvers is defined as

$$
\begin{aligned}
& \Delta v_{t_{p}}=(n a \eta / 4)[\delta a / a+\delta e /(1+e)] \\
& \Delta v_{t_{a}}=(n a \eta / 4)[\delta a / a-\delta e /(1-e)]
\end{aligned}
$$

where $\eta=\sqrt{1-e^{2}}$.

By perfoming these five maneuvers over the course of an orbit, the desired set of orbital elements for a spacecraft can be achieved.

\subsection{Back-Propagation Simulation Results}

To verify the practical application of the proposed equations of relative motion, along with the proposed idea of back-propagation, a simulation was performed. The simulation follows the scenario oulined previously in this chapter. The orbital elements for the leader and follower spacecraft are given in Table 4.1.

Table 4.1: Orbital Elements for Closed-Loop Guidance and Control Simulation

\begin{tabular}{|l|c|c|c|}
\hline Orbital Element & Desired Leader Final & Desired Follower Final & Follower Initial \\
\hline Semi-major Axis (km) & 15,000 & 15,000 & 15,010 \\
Eccentricity & 0.2 & 0.201 & 0.21 \\
Inclination (deg) & 59 & 59 & 58 \\
Argument of Perigee (deg) & 20 & 20 & 20.3 \\
RAAN (deg) & 84 & 84 & 83.5 \\
True Anomaly (deg) & 3 & 3 & 2 \\
\hline
\end{tabular}

In this scenario, the desired final configuration must be achieved ten orbital periods from the initial time, and only the follower is controlled. As previously discussed, there exists two potential methods of achieving the final configuration, both using the same impulsive control method. In Case 1, the follower is uncontrolled until closer to the final time, at which point, based on the current orbital elements, the impulsive control law is applied. In Case 2, back-propagation is used to determine a set of ideal orbital 
elements at the initial time, the impulsive control law is immediately applied, and the spacecraft drift into the desired formation for the rest of the simulation time. Although in each case a similar maneuver is used, the magnitude of impulsive control inputs applied in each case will vary as a result of the orbital elements varying with time due to $J_{2}$. In other words, the objective of Case 2 and the back-propagation method is to take advantage of the effects of the $J_{2}$ perturbation.

It should also be noted here that, in an effort to correct the orbital elements more accurately, two sets of tangential in-plane maneuvers were applied. The magnitudes of the second set of maneuvers was calculated based on the orbital element errors after the first set of maneuvers was applied. Only one set of radial maneuvers were applied, and these maneuvers take place with the second tangential maneuvers, when the semimmajor axis and eccentricity have already been corrected to be closer to the desired values. The magnitude of the impulsive maneuvers are summarized in Table 4.2. Additionally, to validate that the spacecraft was being controlled correctly, the orbital element errors for Case 1 and 2 for the follower spacecraft are provided in Figs. 4.3 and 4.4 .

Table 4.2: Comparison of required $\Delta v$ for the Two Simulation Cases

\begin{tabular}{|l|c|c|}
\hline Maneuver & Case 1 & Case 2 \\
\hline$\Delta v_{h}(\mathrm{~m} / \mathrm{s})$ & 140.6 & 149.7 \\
$\Delta v_{r_{p}, 1}(\mathrm{~m} / \mathrm{s})$ & 0 & 0 \\
$\Delta v_{r_{a}, 1}(\mathrm{~m} / \mathrm{s})$ & 0 & 0 \\
$\Delta v_{t_{p}, 1}(\mathrm{~m} / \mathrm{s})$ & 10.1 & 10.2 \\
$\Delta v_{t_{a}, 1}(\mathrm{~m} / \mathrm{s})$ & 13.5 & 13.5 \\
$\Delta v_{r_{p}, 2}(\mathrm{~m} / \mathrm{s})$ & 42.0 & 11.4 \\
$\Delta v_{r_{a}, 2}(\mathrm{~m} / \mathrm{s})$ & 41.4 & 10.2 \\
$\Delta v_{t_{p}, 2}(\mathrm{~m} / \mathrm{s})$ & 1.2 & 1.3 \\
$\Delta v_{t_{a}, 2}(\mathrm{~m} / \mathrm{s})$ & 0.2 & 0.2 \\
\hline Total $\Delta v(\mathrm{~m} / \mathrm{s})$ & 249.0 & 196.5 \\
\hline
\end{tabular}

As shown in Figs. 4.3 and 4.4, the control maneuvers successfully correct the orbital elements. There are still errors in each orbital element after ten orbits, but these errors are small compared to the initial errors. In a real-world scenario it would be more realistic to perform multiple sets of maneuvers (more than two) over the course of a few orbits to ensure the orbital elements are corrected perfectly. It also should be noted that, as can be seen in Figs. 4.3 and 4.4, each orbital element does vary somewhat with time due to $J_{2}$ affecting the orbital elements through periodic 

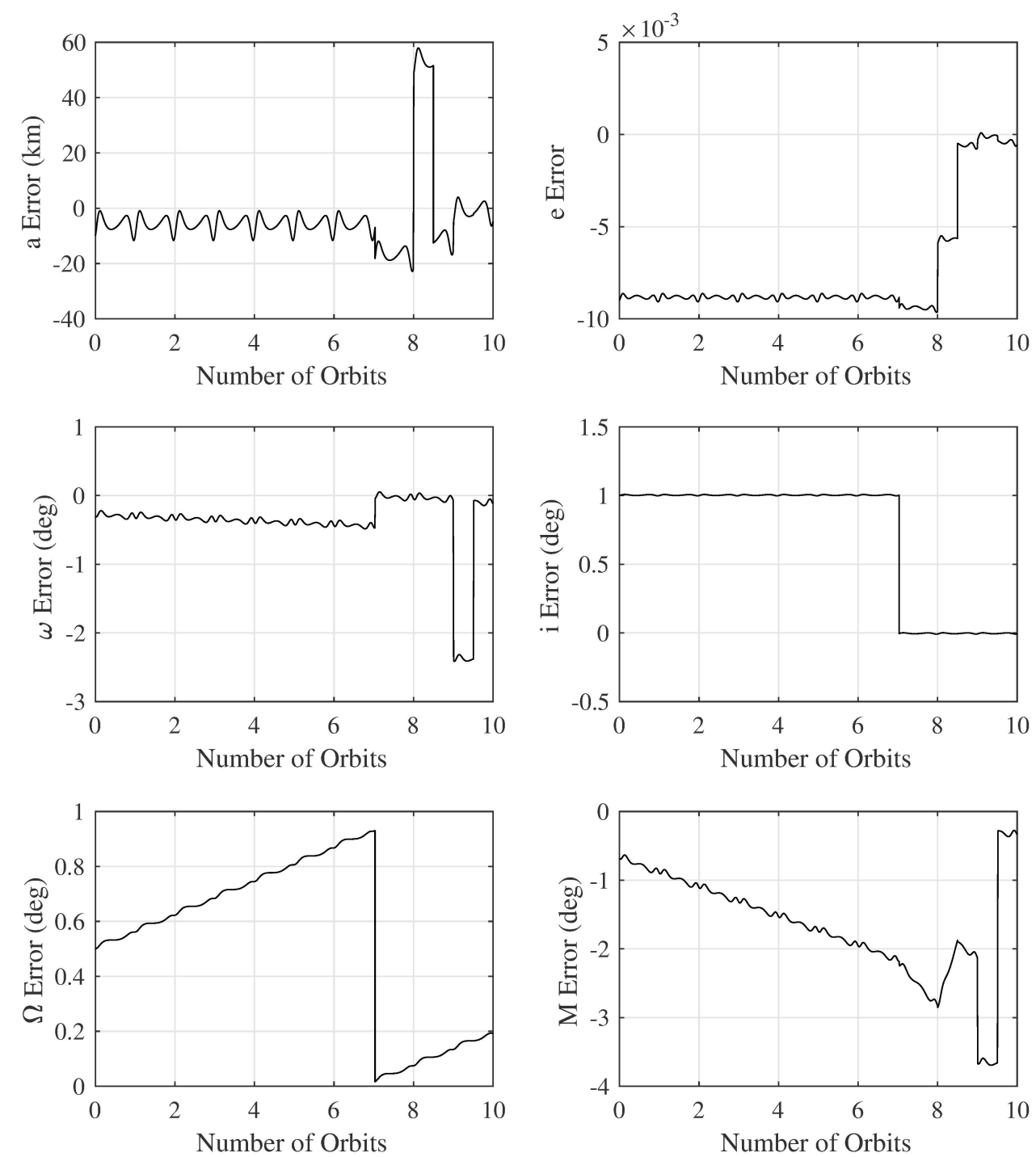

Figure 4.3: Closed-loop back-propagation guidance and impulsive control performance validation for Case 1 . 

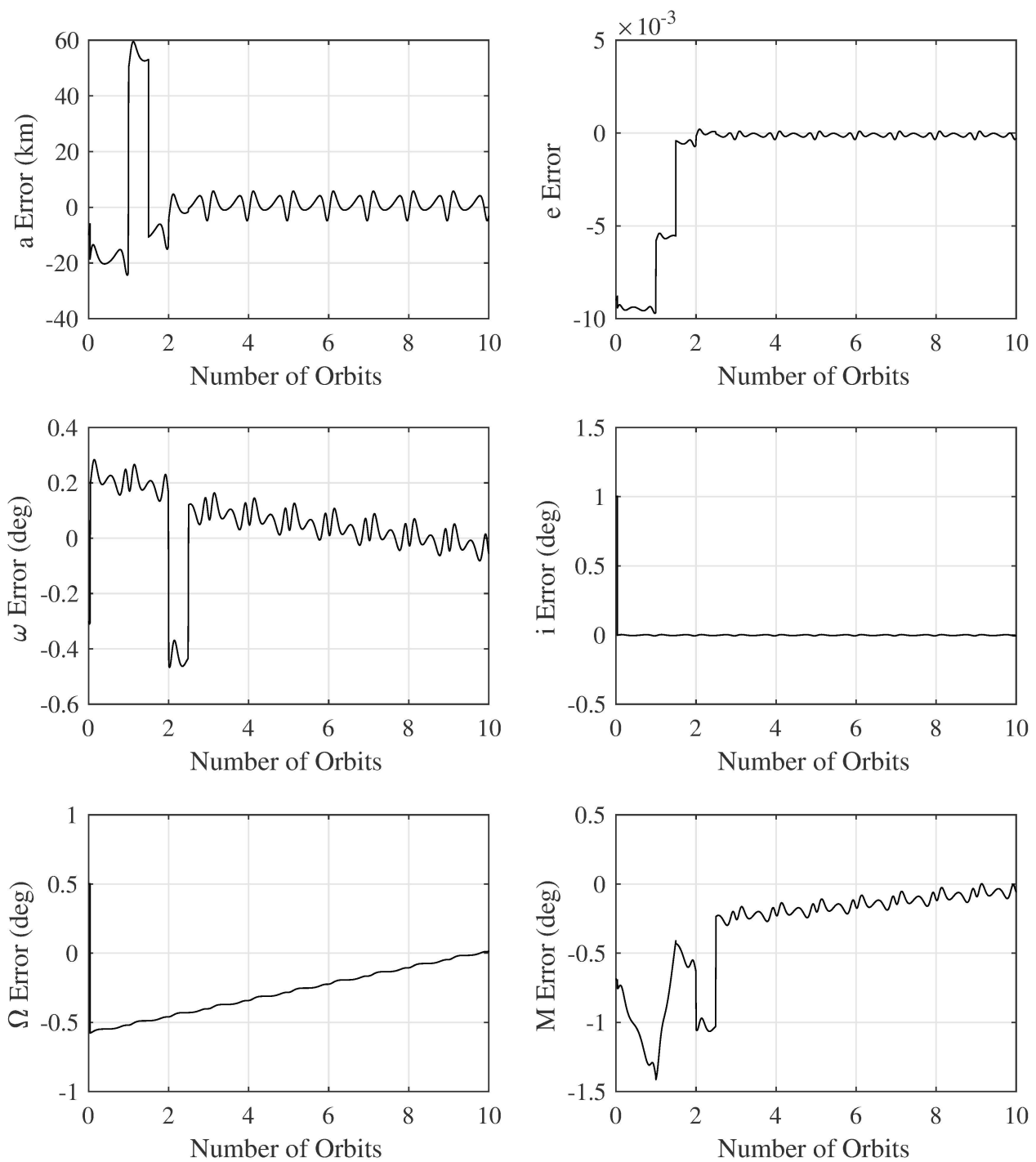

Figure 4.4: Closed-loop back-propagation guidance and impulsive control performance validation for Case 2 . 
variations.

Table 4.2 shows that Case 2 uses significantly less $\Delta v$ to accomplish the same reconfiguration maneuver. From this table, it is clear that, although there might be small differences between the $\Delta v$ for each maneuver, Case 2's decreased fuel useage is mainly a result of much smaller radial $\Delta v$ maneuvers. This is expected, as the $\Delta v$ differences are a function of the effects of the $J_{2}$ perturbation on the orbital elements. For most of the orbital elements, $J_{2}$ has very little effect over the course of ten orbits, or no effect at all. However, the difference between desired and actual mean anomaly does vary significantly over the course of a few orbits, due both to a difference in period of the spacecraft, and a relative drift between the spacecraft due to $J_{2}$. The mean anomaly difference $(\delta M)$ directly affects the radial $\Delta v$ maneuvers.

It should be noted that the $\Delta v$ magnitudes shown in Table 4.2 are of a large magnitude for typical spacecraft. This is a result of the relatively large orbital element errors which are being corrected. This type of large reconfiguration maneuver would be uncommon for most missions, but could be likened to the bringing of two spacecraft in formation to close proximity by corrected their orbital element differences after they have been deployed from a launcher. In any case the situation which is presented demonstrates the potential benefits of the proposed guidance strategy, and the same benefits would apply to closer proximity maneuvers.

It should be noted that in this particular scenario the back-propagation method is more efficient mainly due to the fact that, based on the initial conditions, the spacecraft would tend to drift apart from each other. In cases where the spacecraft would initially drift closer to their desired formation, the improvement from using the back-propagation guidance will almost certainly be much less, and in some cases the better option may actually be to perform the maneuvers later in time without using this guidance strategy. Ideally, spacecraft in formation would be able to plan multiple potential maneuvers based on their predicted motion, and choose the most efficient option.

Overall, the numerical simulation results successfully demonstrate that the backpropagation method for performing reconfiguration maneuvers of spacecraft in formation can be successfully integrated with a impulsive controller for reconfiguration 
purposes. Additionally, the simulation demonstrates a potential application of the back-propagation guidance method and the proposed equations of relative motion, validating that these novel methods can be used to plan more efficient maneuvers. 


\section{Chapter 5}

\section{Continuous Guidance and Control Applications}

The objective of this chapter is to explore some applications of the proposed analytical equations for spacecraft formation motion with a time-continuous PD controller. In these applications, the newly-derived equations are used as the guidance system for the follower spacecraft in formation. Results are presented showing the potential benefits of the novel guidance system, both for formation keeping and reconfiguration maneuvers when used in conjuction with the back-propagation method.

\section{$5.1 \quad$ Introduction}

In this section, two examples of the use of the proposed analytical equations for spacecraft relative position in a closed-loop control scenario will be discussed. These scenarios differ from the results found in Sec. 4.3 in that they will use continuous and not impulsive control. This is more realistic for typical formation keeping operations.

The navigation system for a spacecraft uses instruments to estimate the actual position of the spacecraft, or in the case of spacecraft formation flying the relative position between the spacecraft. In the guidance and control (G\&C) simulations presented in this thesis no realistic navigation system is included, as this is beyond the scope of this research. Instead the spacecraft dynamics are used to obtain the actual position based on the numerical simulator presented in Chap. 2.7. This actual position signal is compared with the desired relative position, or in other words the guidance system of the spacecraft. The error between these signals can then be combined with a time-continuous controller to calculate control inputs such that the error is corrected and the true relative position from the dynamics model matches the desired relative position of the guidance.

The work presented in this chapter is linked by a common controller, the simple PD controller that will be defined in Sec. 5.2: Closed-Loop GECC Simulation, along 
with a description of the full spacecraft guidance and control simulation. Section 5.3: GEC with Clohessy-Wiltshire Equations presents baseline guidance and control results obtained using the G\&C simulator with the Clohessy-Wiltshire equations [6], previously defined in Sec. 2.8 as a guidance system. From here, two separate guidance and control scenarios will be investigated. In Sec. 5.4: Proposed Analytical Solution Guidance System the proposed analytical equations for spacecraft relative motion derived in Chap. 3 are implemented as the guidance system for a full G\&C scenario. Sec. 5.5: Back-Propagation With Continuous Control continues to use the proposed equations for guidance, but this time making use of the back propagation concept introduced in Chap. 4.

\subsection{Closed-Loop G\&C Simulation}

A guidance system may be judged on its accurracy by comparing it with the true motion of the spacecraft, but ultimately they should be validated along with control and spacecraft dynamics in a closed-loop guidance and control (G\&C) scenario.

In this thesis, a full G\&C scenario is set up in MATLAB/Simulink. A simple block diagram showing the basic set up of this G\&C simulation is shown in Fig. 5.1 below.

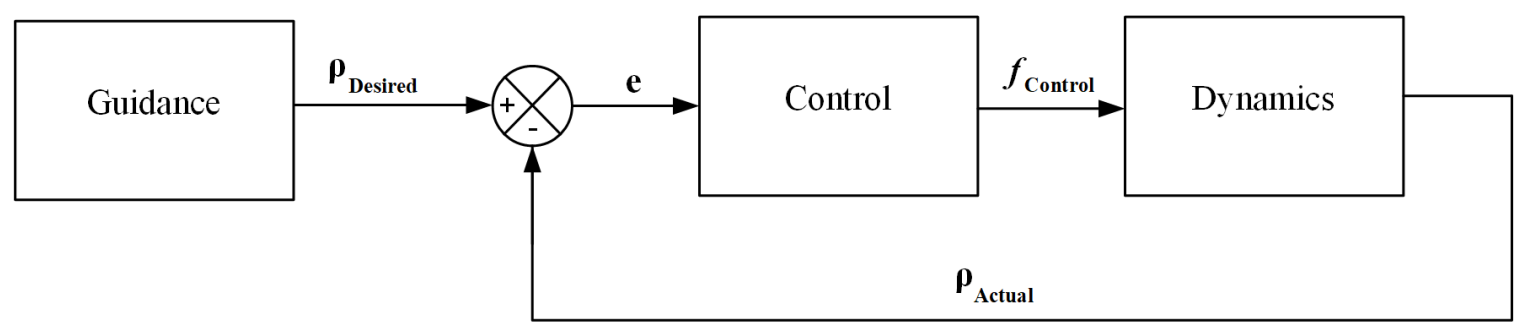

Figure 5.1: Schematic diagram of G\&C simulation.

The desired relative position from the guidance block is compared with the actual position of the follower relative to the leader spacecraft provided by the dynamics block, which uses the numerical simulator defined in Sec. 2.7. Note that in this case the numerical simulator was implemented in Simulink with a constant time step of 1 second. The resulting error found by subtracting the true position from the desired 
position is fed into a PD controller, which outputs an $\mathrm{x}, \mathrm{y}$ and $\mathrm{z}$ control acceleration to be imparted on the follower spacecraft, calculated using the following equations

$$
\begin{aligned}
& a_{x}=K_{p} e_{x}+K_{d} \dot{e}_{x} \\
& a_{y}=K_{p} e_{y}+K_{d} \dot{e}_{y} \\
& a_{z}=K_{p} e_{z}+K_{d} \dot{e}_{z}
\end{aligned}
$$

where the $e$ and $\dot{e}$ terms represent the position and velocity error in each direction respectively, and $K_{p}=0.01$ and $K_{d}=0.1$ are the controller constants (the controller was tuned by trial and error). The resulting control input is taken into account by adding the acceleration to the two-body equation of motion (along with the $J_{2}$ perturbing acceleration). Thus, the closed-loop G\&C system continuously controls the follower spacecraft, maneuvering it to achieve the desired formation. Note that the simple linear control law used in this section and the use of trial and error for tuning the controller reflect the scope of this thesis. The goal of this chapter is not to focus on the advanced or even realistic control of a spacecraft. Instead, the goal is to

demonstrate that the proposed equations for guidance can be applied in a closed-loop control scenario, and to explore the possible benefits of the proposed equations when used as a guidance system.

This $\mathrm{G} \& \mathrm{C}$ simulation is easy to adjust for the different guidance systems that will be compared later in the thesis, as any set of equations which output the $\mathrm{x}, \mathrm{y}$ and $\mathrm{z}$ position of the follower spacecraft with respect to the leader spacecraft may be substituted into the guidance block.

\subsection{G\&C with Clohessy-Wiltshire Equations}

The Hill-Clohessy-Wiltshire (HCW) equations previously defined in Eqs. 2.37-2.39 of Sec. 2.8 are easily adapted to a guidance system, and this guidance system will be used to obtain baseline $\mathrm{G} \& \mathrm{C}$ results for comparison with methods using the proposed equations derived in this thesis. The HCW equations define a time-varying $\mathrm{x}, \mathrm{y}$ and 
z position of the follower spacecraft relative to the leader. Through the careful choice of initial conditions used in these equations, a variety of useful formations can be defined, including along-track, in-plane elliptical, and projected-circular formations. For now, a simple in-plane elliptical formation will be considered, defined by a small difference in eccentricity between the orbits of the leader and follower spacecraft.

As previously mentioned in Sec. 1.3, due to simplifying assumptions made in their derivation, the HCW equations are only able to predict relative motion accurately in unperturbed, circular orbits. As a result, the HCW model makes for a very effective guidance system only if the spacecraft in formation are on such restricted orbits. In this section, two cases using the HCW equations for guidance will be compared. In the first case, the $J_{2}$ perturbation is removed from the numerical simulator and the eccentricity of the orbits is set to 0 . Table 5.1 shows the desired and initial orbital elements for each spacecraft for this case. Note that the follower's initial orbital elements are defined such that there are small errors in the orbital elements, allowing the simulation to test the ability of the G\&C system to correct initial errors and then maintain the desired formation.

Table 5.1: Orbital Elements for Continuous G\&C System (Circular Case)

\begin{tabular}{|l|c|c|c|}
\hline Orbital Element & Desired Leader Final & Desired Follower Final & Follower Initial \\
\hline Semi-major Axis (km) & 15,000 & 15,000 & $15,000.1$ \\
Eccentricity & 0 & 0.00001 & 0 \\
Inclination (deg) & 59 & 59 & 59.003 \\
Argument of Perigee (deg) & 20 & 20 & 19.999 \\
RAAN (deg) & 84 & 84 & 83.998 \\
True Anomaly (deg) & 3 & 3 & 2.9996 \\
\hline
\end{tabular}

Figure 5.2 shows that the implementation of the HCW guidance in the G\&C system works well, with part (b) of the figure showing that the initial errorr in each direction is reduced to approximately zero within about 110 seconds. From that point onwards the desired formation is maintained. Figure 5.3 shows the control inputs with time, with part (a) showing the relatively large control inputs over the first few hundred seconds required to correct the initial errors, and part (b) showing the much smaller control inputs which maintain the desired relative motion over six orbital periods. As can be seen, once the initial errors are corrected very little control input is required. This makes sense, as once the initial errors are corrected, the 
spacecraft should have reached the desired relative motion, and in this unperturbed, circular orbit case there is nothing to push the spacecraft off of this desired motion.

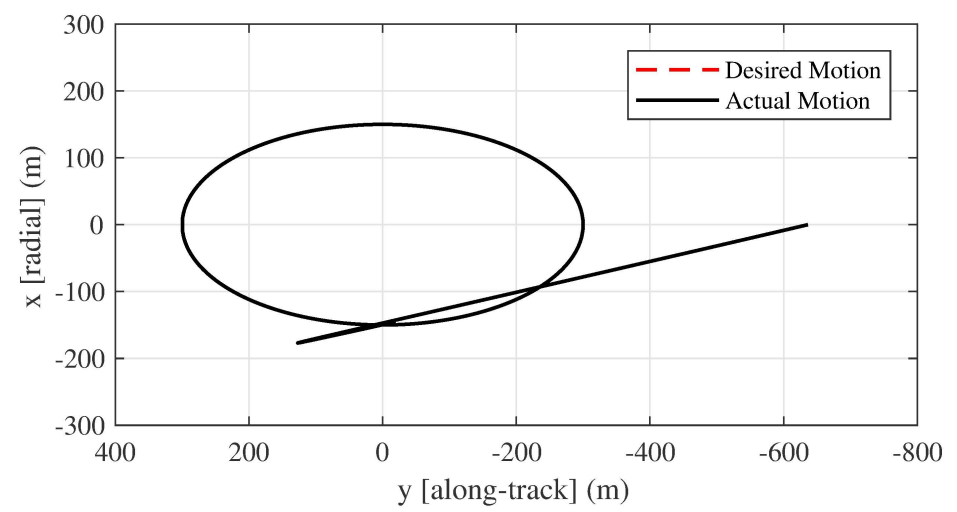

(a) Desired and actual in-plane motion

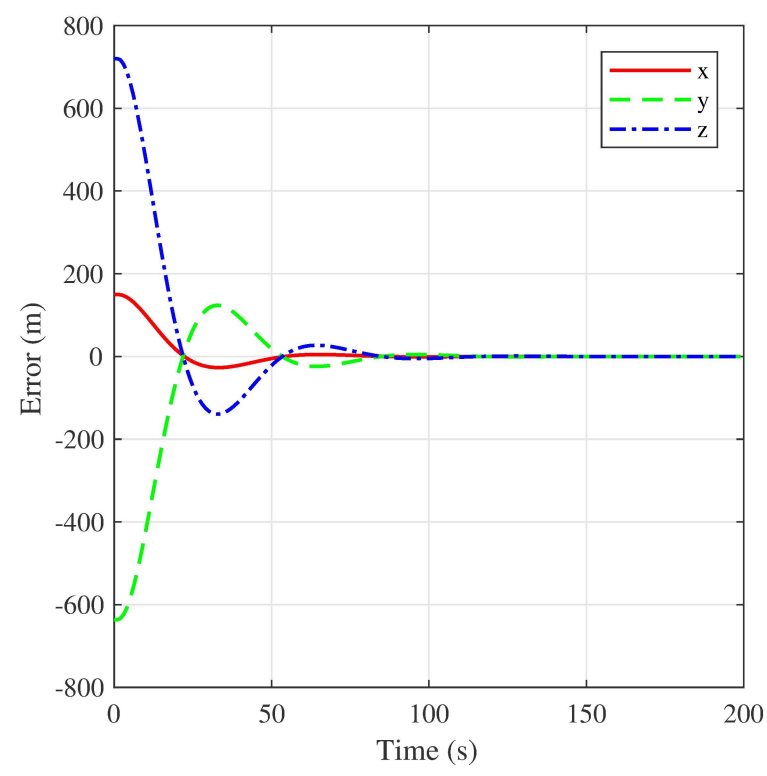

(b) Position error for first 200 seconds of simulation

Figure 5.2: Validation of control effectiveness for G\&C system using HCW model in an unperturbed, circular orbit.

However, assuming no perturbations is unrealistic in real mission scenarios, and practical missions may require a non-zero eccentricity. Case 2 aims to evaluate how the HCW guidance performs in a more realistic situation by adding the acceleration from the $J_{2}$ perturbation back into the numerical simulator, and by using a more elliptical orbit. Table 5.2 shows the desired and initial orbital elements for the the 


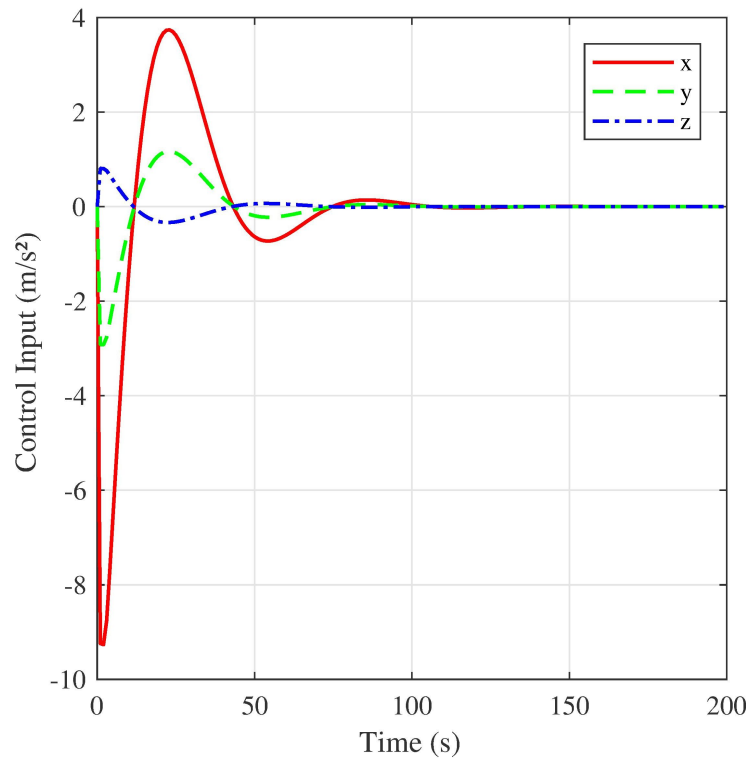

(a) Control input for first 200 seconds of simulation

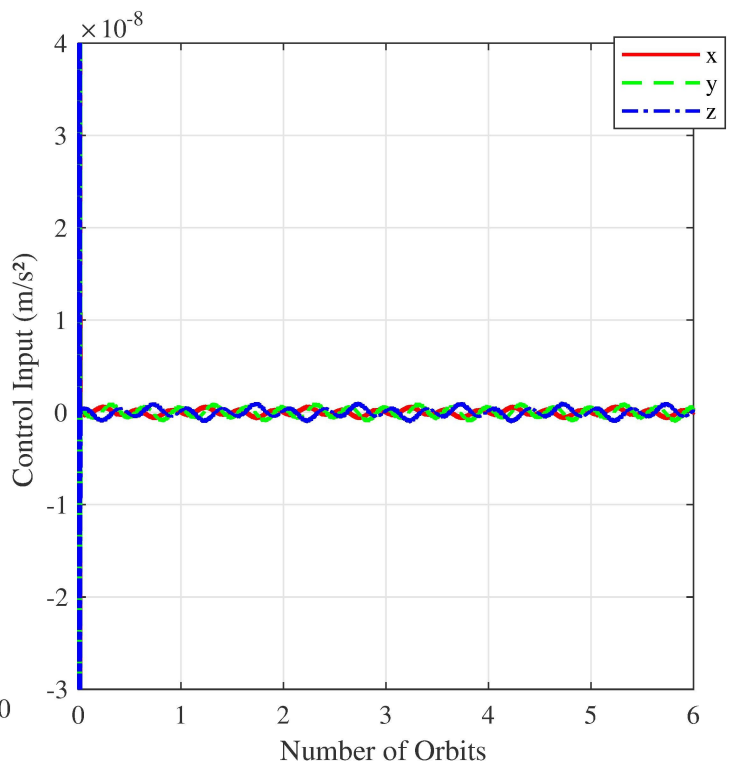

(b) Control input for entire simulation

Figure 5.3: Control inputs for G\&C system using HCW model in an unperturbed, circular orbit.

spacecraft in moderately elliptical orbits with an eccentricity of 0.2 .

Table 5.2: Orbital Elements for Continuous G\&C System (Elliptical Case)

\begin{tabular}{|l|c|c|c|}
\hline Orbital Element & Desired Leader Final & Desired Follower Final & Follower Initial \\
\hline Semi-major Axis (km) & 15,000 & 15,000 & $15,000.1$ \\
Eccentricity & 0.2 & 0.20001 & 0.2 \\
Inclination (deg) & 59 & 59 & 59.003 \\
Argument of Perigee (deg) & 20 & 20 & 19.999 \\
RAAN (deg) & 84 & 84 & 83.998 \\
True Anomaly (deg) & 3 & 3 & 2.9996 \\
\hline
\end{tabular}

Figure 5.4 shows much the same results as Fig. 5.2, the controller still effectively reduces the initial error to zero. This makes sense, as the initial error is the same, and the controller handles it the same way. However, Fig. 5.5 shows a very different result from 5.3. Although part (a) looks the same, once again as a result of the initial relatively large errors being approximately the same, part (b) shows that much larger control inputs are required to maintain the desired formation. This is because the spacecraft no longer naturally stay in their desired formation. The $J_{2}$ perturbation and the eccentricity of the orbits means that the spacecraft follow a more complicated relative motion than the simple in-plane elliptical formation described by the HCW 
equations, and a significant amount of control input is required to force the follower spacecraft to maintain that formation.

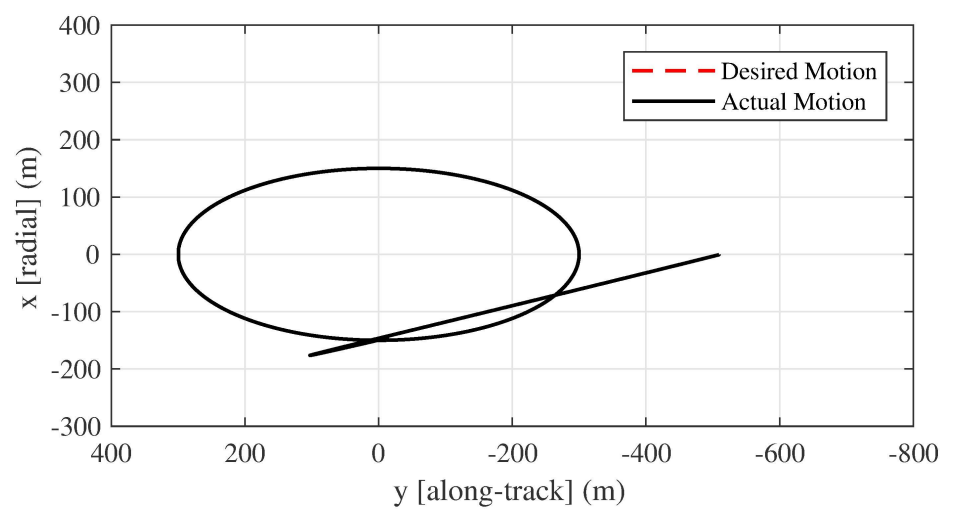

(a) Desired and actual in-plane motion

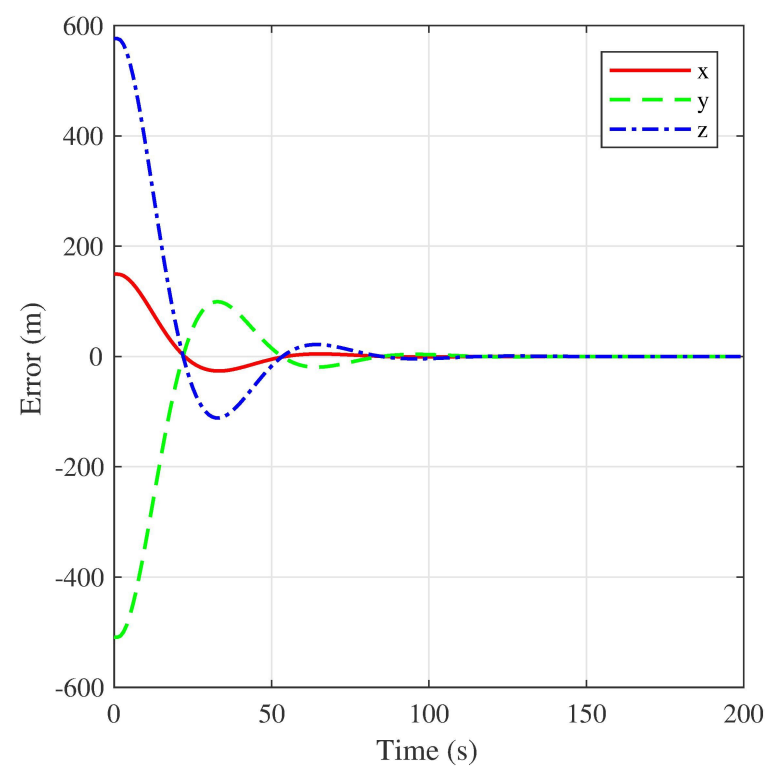

(b) Position error for first 200 seconds of simulation

Figure 5.4: Validation of control effectiveness for G\&C system using HCW model in a $J_{2}$-perturbed, elliptical orbit.

\subsection{Proposed Analytical Solution Guidance System}

This practical application of the newly-derived equations for relative motion involves the use of the analytical expressions as the guidance system in a formation flying G\&C scenario. In other words, the proposed equations will be used to define the 


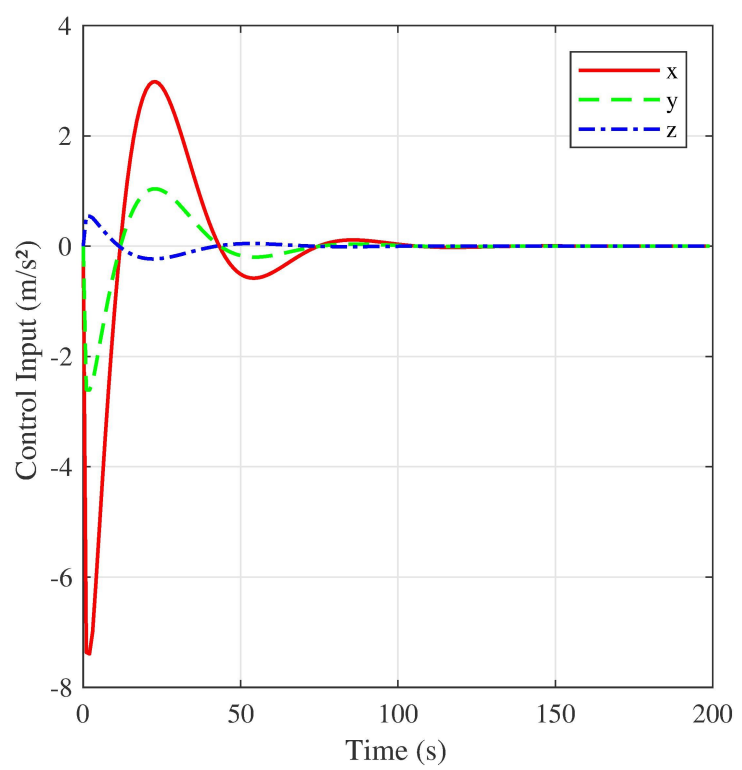

(a) Control input for first 200 seconds of simulation

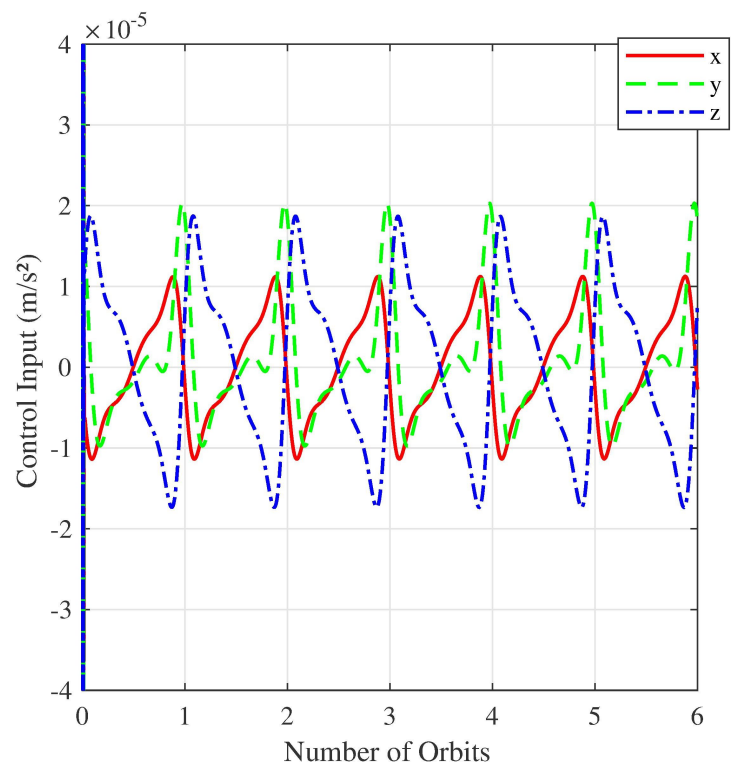

(b) Control input for entire simulation

Figure 5.5: Control inputs for G\&C system using $\mathrm{HCW}$ model in a $J_{2}$-perturbed, elliptical orbit. 
desired spacecraft relative position, much like the Hill-Clohessy-Wiltshire Equations were used in the previous section. The same PD controller and numerical simulator used in the previous section are once again used. The goal of this section is to observe the use of the proposed equations as a guidance system in a continuous G\&C scenario.

As was seen in the previous section, using the HCW equations for guidance may imply a significant amount of $\Delta v$ being used to maintain the formation if the spacecraft are in $J_{2}$-perturbed, eccentric orbits. This is not a problem when using the proposed equations, as they take into account the $J_{2}$ perturbation and the eccentricity of the orbit by definition. Instead of setting up an elliptical, non-drifting formation, the formation will be irregular in shape depending on the eccentricity of the orbits of the spacecraft in formation, and will drift as a result of $J_{2}$. Although this irregular motion may be undesirable compared to the less complicated motion found when using the HCW model it has the advantage of being considerably more true to the natural, passive, spacecraft motion. The spacecraft motion is predictable based on the equations, and will require significantly less control input to maintain when perturbations are used in the numerical simulator.

The implementation of this G\&C system is quite simple. The HCW equations are simply replaced by the proposed equations, being propagated forward in time as described in Chap. 3. The closed-loop control simulation with continuous control was once again run using the orbital elements from Table 5.2.

Figure 5.6 (b) shows very similar results to Figs. 5.2 and 5.4. Once again this is expected, as the initial errors are still similar, and the controller applies large accelerations to reduce this initial error to zero. However, Fig. 5.6 (a) shows the drifting motion of the formation which results from the inclusion of $J_{2}$ in the guidance. The more subtle effect of the non-zero eccentricity can also be seen, with the formation no longer being perfectly elliptical, but more of a bent ellipse shape. Although the resulting motion is a little bit different from the motion seen while using the $\mathrm{HCW}$ guidance, the G\&C system still quickly converges to the desired motion from the guidance system, and maintains this formation for six orbits.

Figure 5.7 (a) shows the same large initial control inputs as in Fig. 5.5 (a). However, comparing part (b) of each of these figures it is clear that the proposed guidance 
system uses about two orders of magnitude smaller control inputs at any given time to maintain the formation. This demonstrates that, although irregular, the predictable motion of the proposed guidance equations can be used to more efficiently maintain a desired formation.

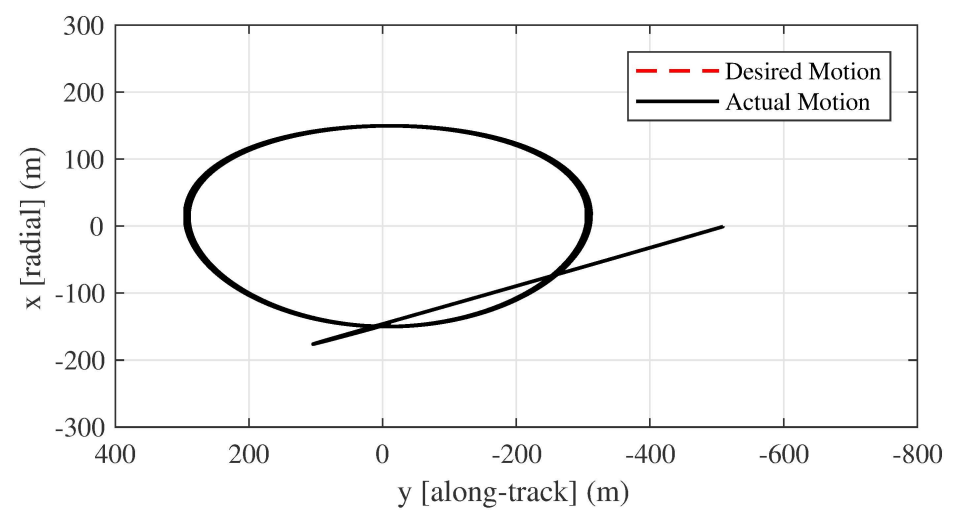

(a) Desired and actual in-plane motion

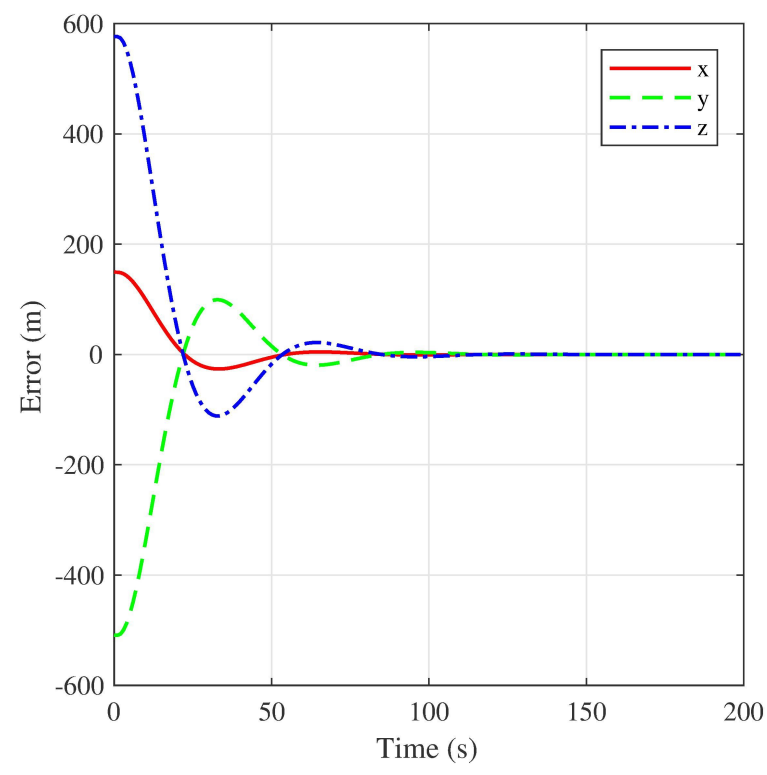

(b) Position error for first 200 seconds of simulation

Figure 5.6: Validation of control effectiveness for G\&C system using proposed analytical equations in a $J_{2}$-perturbed elliptical orbit.

To demonstrate the difference between the HCW model and the proposed equations guidance, Fig. 5.8 was generated. In this figure the effects of eccentricity can be seen a bit more clearly when comparing the HCW model (which assumes the orbits 


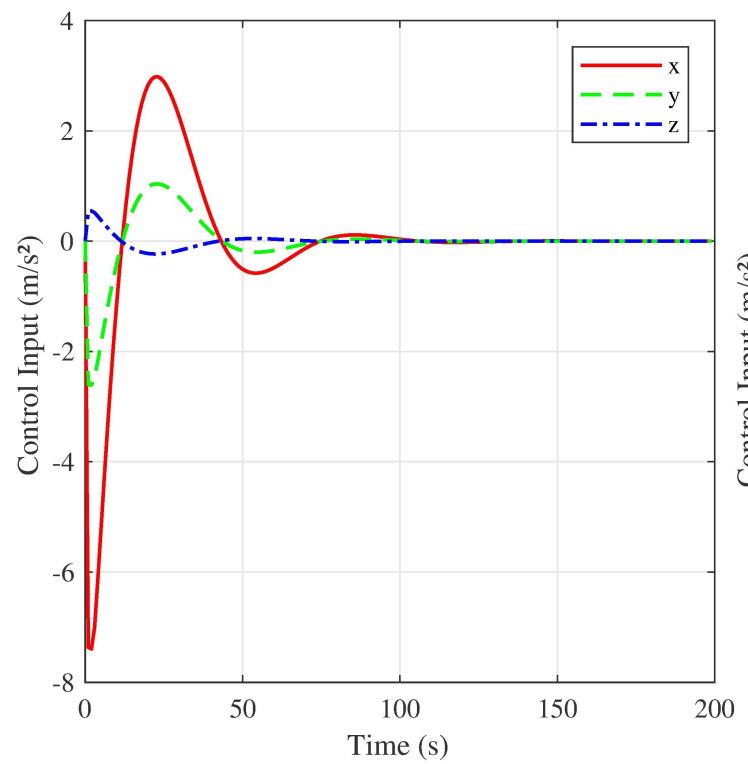

(a) Control input for first 200 seconds of simulation

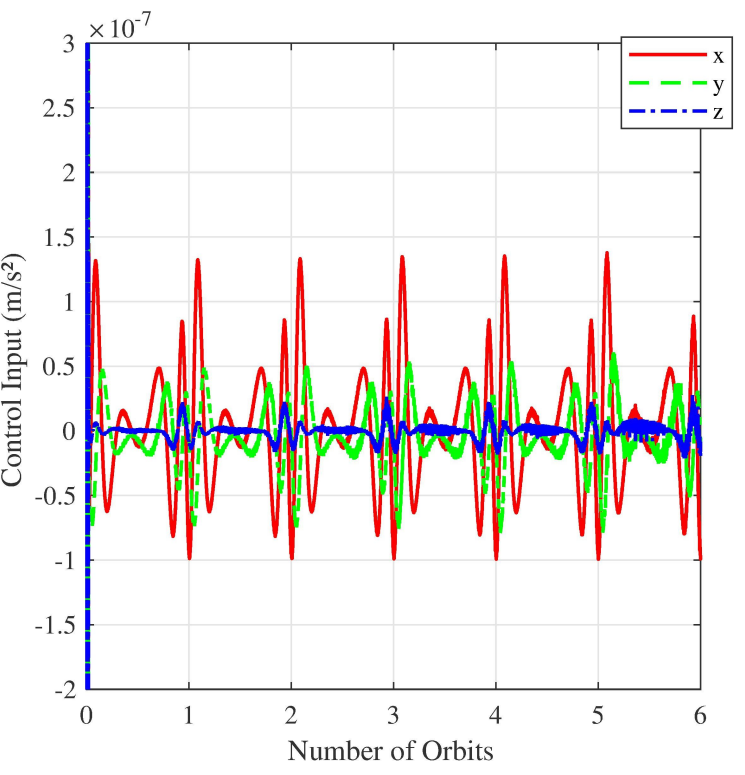

(b) Control input for entire simulation

Figure 5.7: Control inputs for G\&C system using proposed analytical equations in a $J_{2}$-perturbed elliptical orbit.

are circular) and the proposed equations.

The irregular shape of formations in highly eccentric orbits is difficult to avoid, as the magnitude of the position vectors, and in general all of the orbital elements, of each spacecraft can change quickly at times, especially near perigee. Therefore, certain formations, for example true in-plane elliptical formations, will always be a difficult task in high eccentricity orbits. Using the proposed equations of guidance is much more fuel efficient, as long as the mission can handle some irregular motion.

The irregular shape of the natural formation motion caused by non-zero eccentricity can be observed at even greater level by using a highly elliptical orbit formation case. The orbital elements for this case can be found in Table 5.3. Figs. 5.9 and 5.10 show the results for the highly elliptical orbit using the HCW model with an in-plane elliptical formation. Once again, the resulting formation is a simple ellipse, but this is far from the natural motion of the spacecraft, and large control inputs are required to keep the spacecraft in this formation. Figure 5.11 (a) shows what the more natural motion of the formation would look like, and Fig. 5.12 (b) shows that once again, significantly less control input is required to maintain this more natural formation. 


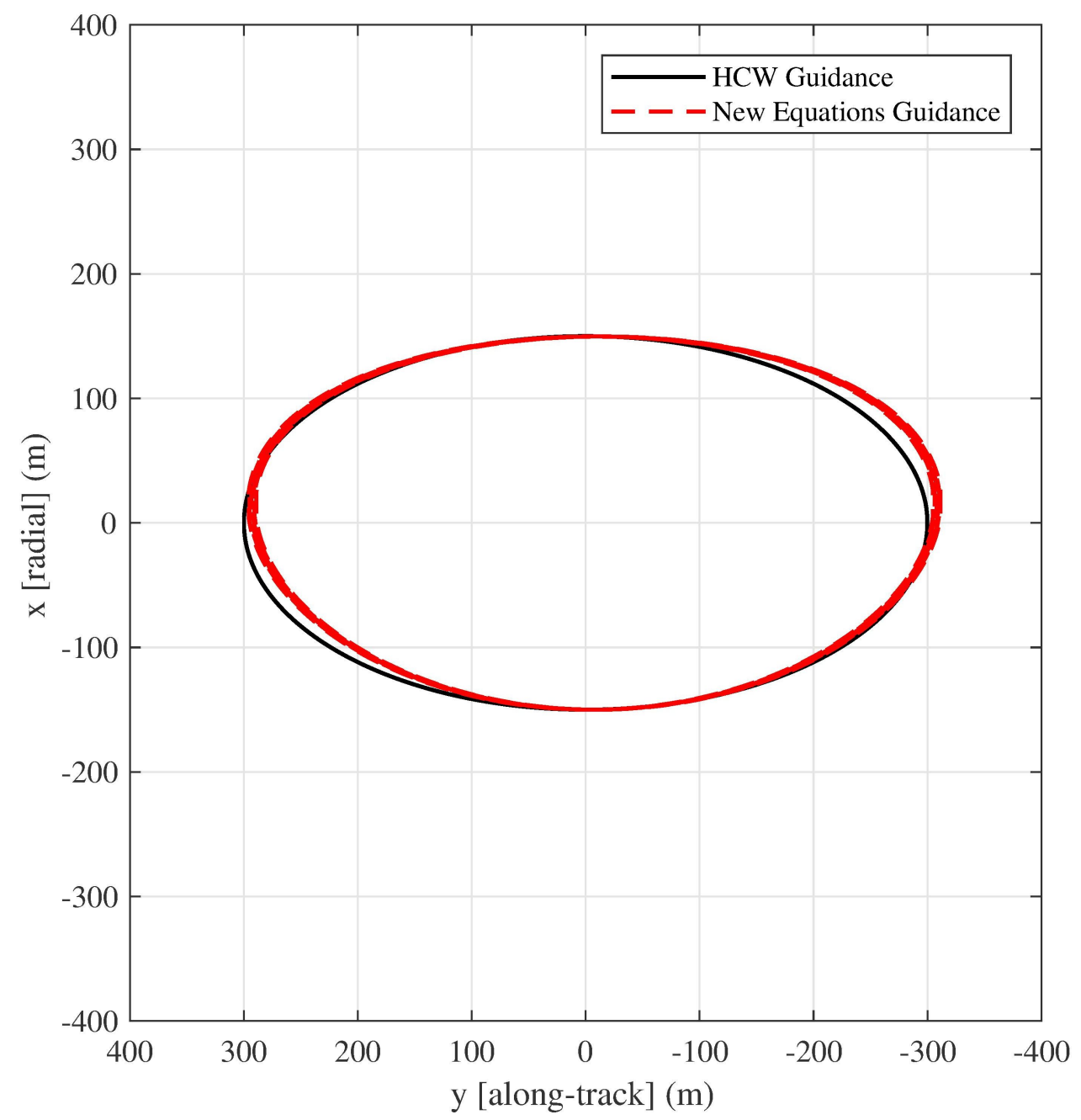

Figure 5.8: Comparison between HCW guidance, and proposed guidance for inplane motion of formation case in Table 5.2.

Table 5.3: Orbital Elements for Continuous G\&C System (Highly Elliptical Case)

\begin{tabular}{|l|c|c|c|}
\hline Orbital Element & Desired Leader Final & Desired Follower Final & Follower Initial \\
\hline Semi-major Axis (km) & 35,000 & 35,000 & $35,000.1$ \\
Eccentricity & 0.8 & 0.80001 & 0.8 \\
Inclination (deg) & 59 & 59 & 59.003 \\
Argument of Perigee (deg) & 20 & 20 & 19.999 \\
RAAN (deg) & 84 & 84 & 83.998 \\
True Anomaly (deg) & 3 & 3 & 2.9996 \\
\hline
\end{tabular}




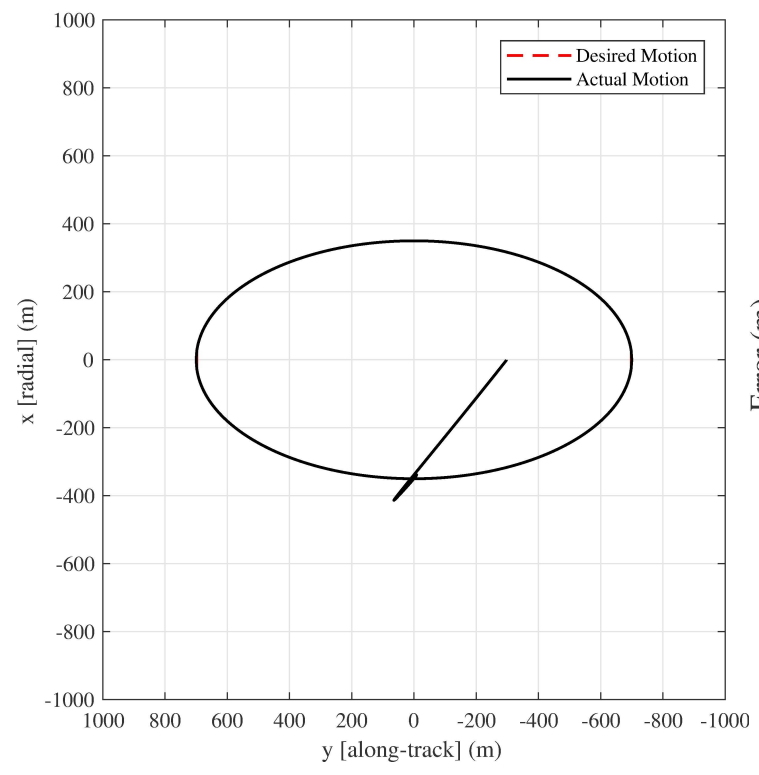

(a) Desired and actual in-plane motion

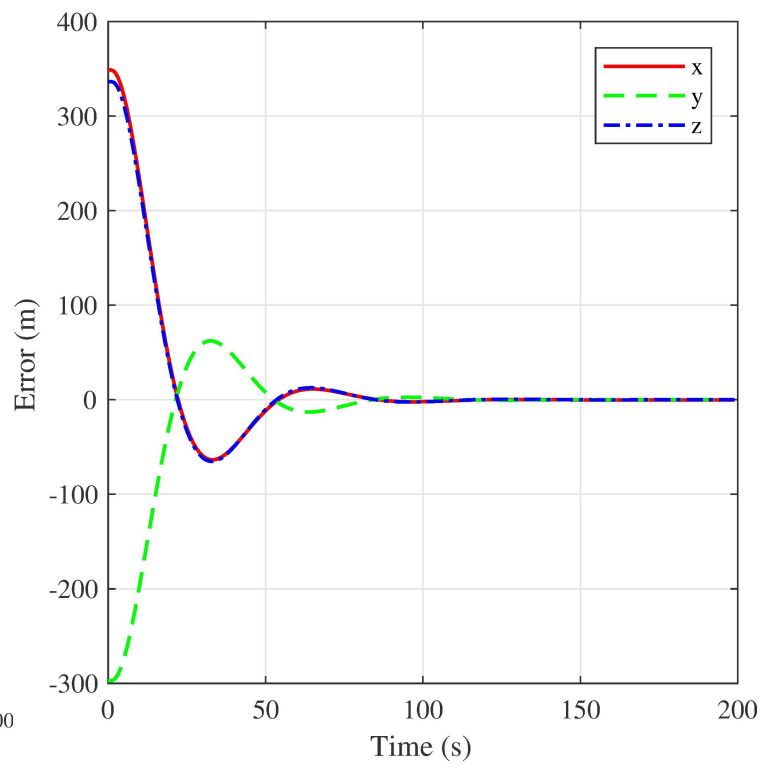

(b) Position error for first 200 seconds of simulation

Figure 5.9: Validation of control effectiveness for G\&C system using HCW model in a $J_{2}$-perturbed, highly elliptical orbit.

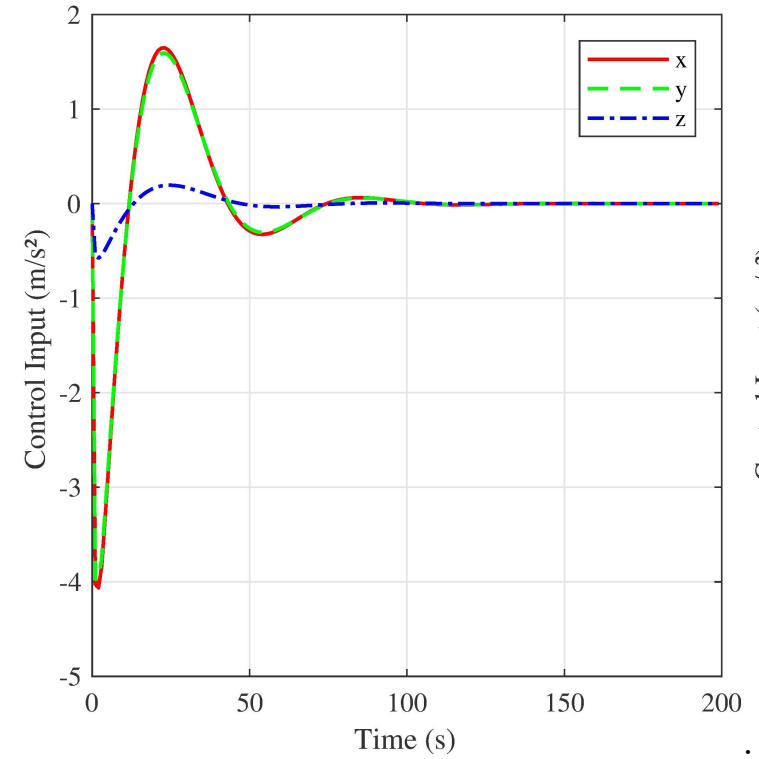

(a) Control input for first 200 seconds of simulation

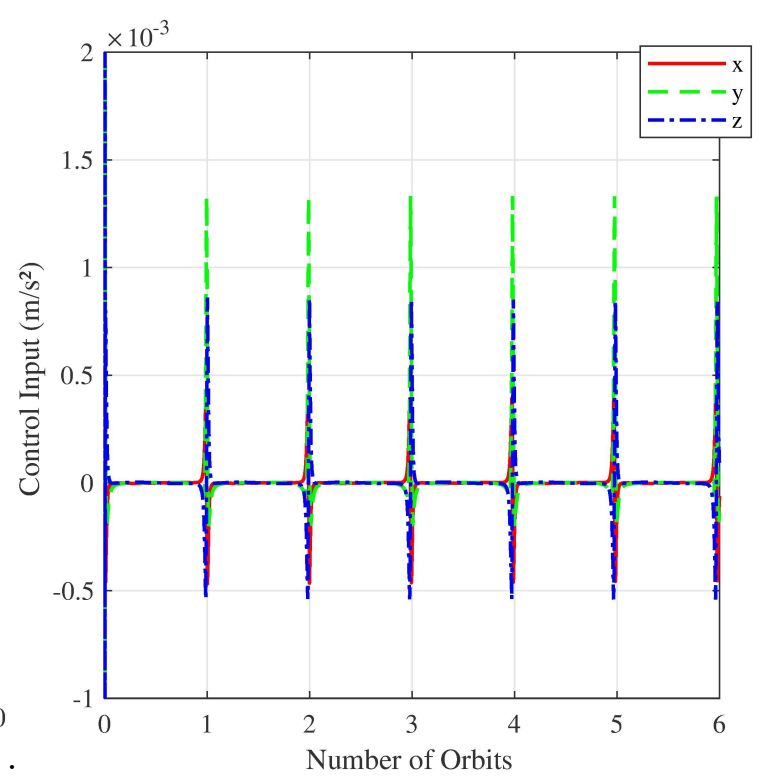

(b) Control input for entire simulation

Figure 5.10: Control inputs for G\&C system using $\mathrm{HCW}$ model in a $J_{2}$-perturbed, highly elliptical orbit. 


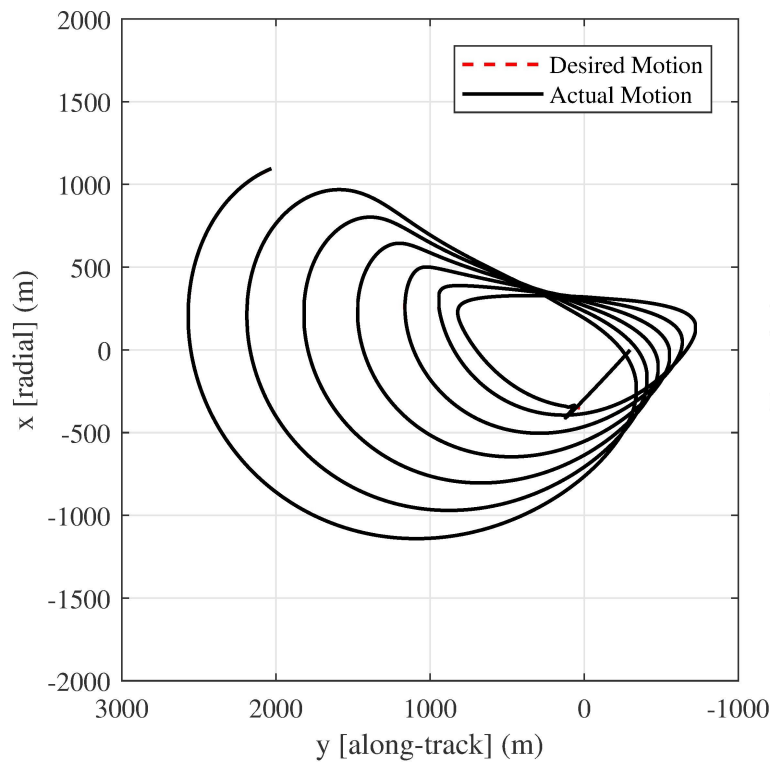

(a) Desired and actual in-plane motion

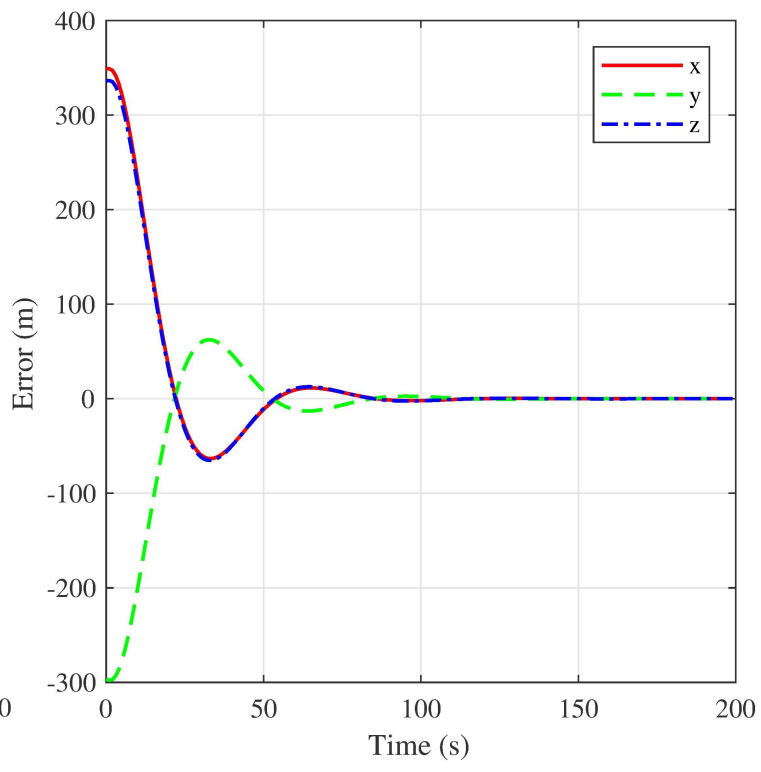

(b) Position error for first 200 seconds of simulation

Figure 5.11: Validation of control effectiveness for G\&C system using proposed analytical equations in a $J_{2}$-perturbed, highly elliptical orbit.

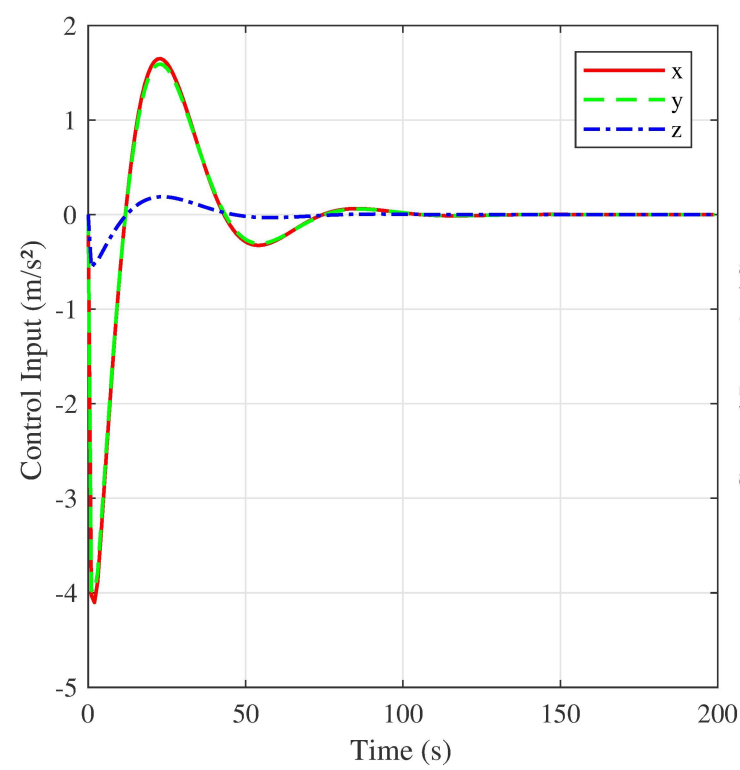

(a) Control input for first 200 seconds of simulation

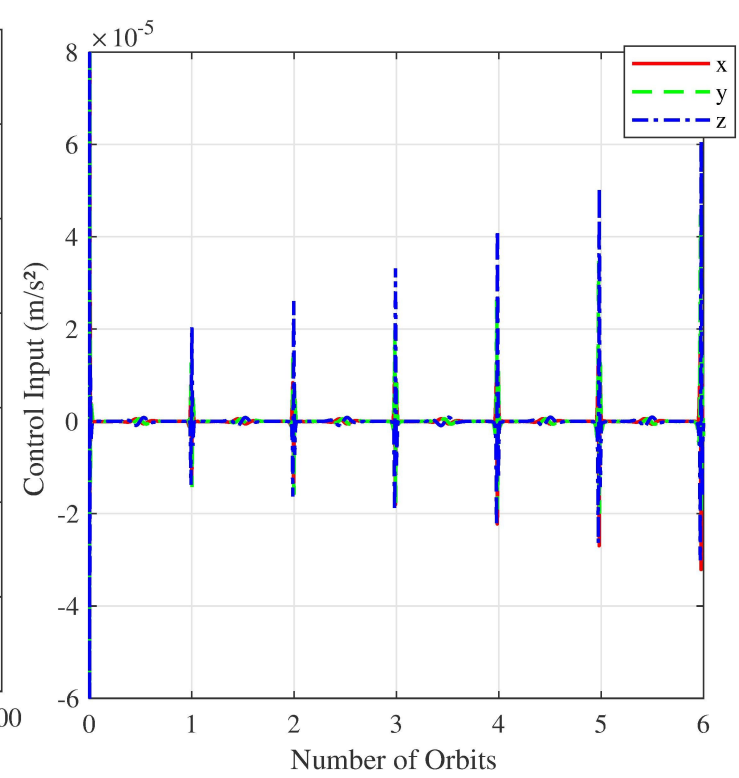

(b) Control input for entire simulation

Figure 5.12: Control inputs for G\&C system proposed analytical equations in a $J_{2}$-perturbed, highly elliptical orbit. 
However, looking at Fig. 5.11 (a), it can be seen that the spacecraft are drifting apart with time. This drifting of the formation is a more serious issue than the irregular shape resulting from an elliptical orbit. This is true for most formations, as typically it is undesirable to have the spacecraft either drifting closer together or further apart. One potential method for addressing the drifting issue is to set up a formation which cancels the drift. Specifically, the drift is caused by the secular variations in the orbital elements caused by $J_{2}$. By the careful choice of orbital elements, this secular variation can be set to be equal for each of the two spacecraft in formation, causing them to drift at the same rate, or in other words causing the relative motion to not drift at all.

This type of orbit is typically called a $J_{2}$-invariant orbit, and work by Schaub et al. [31, 32] can be used to establish such an orbit.

The $J_{2}$-invariant orbit theory states that there are two conditions which should be met to ensure that the drift from the $J_{2}$ perturbation is equal for both spacecraft. These conditions are expressed as differences between the leader and follower orbital elements as follows

$$
\begin{gathered}
\delta \eta=-\frac{\eta^{\prime}}{4} \tan \left(i^{\prime}\right) \delta i \\
\delta a=2 D a^{\prime} \delta \eta
\end{gathered}
$$

where

$$
D=\frac{J_{2}}{2 a^{\prime 2} \eta^{\prime 5}}\left(4+3 \eta^{\prime}\right)\left(1+5 \cos ^{2}\left(i^{\prime}\right)\right)
$$

and where the eccentrity is related to $\eta$ as follows

$$
\eta=\sqrt{1-e^{2}}
$$

These conditions are imposed in mean (not osculating) orbital element space, and result in a small change in three of the desired orbital elements for the chaser spacecraft given in Table 5.3. The eccentricity difference of 0.00001 was maintained as the mean difference, and is used to determine the difference in the initial mean 
semimajor axis and inclination. These were then all translated back to osculating space, and used to initialize the guidance in the G\&C simulation. Using Eqs. (5.4) and (5.5) the three updated osculating orbital elements are $e=0.800010039, a=$ $35000.0126 \mathrm{~km}$ and $i=59.00305$ degrees for the chaser spacecraft.

Running the full G\&C simulation with these updated orbital elements results in Figs. 5.13 and 5.14. Figure 5.14 (a) shows that, although there is still some small drift between the spacecraft likely a result of the assumptions using the proposed equations (for example, neglecting $J_{2}$-induced long-periodic variations in the orbital elements), most of the drift between the spacecraft is eliminated. We are left with an irregularly shaped, flattened elliptical relative motion which, based on Fig. 5.14 (b), requires little control input to maintain. Once again, this irregular shape may be undesirable compared to the even ellipse that results from the HCW model, but the motion is still predictable, and requires much less control input to maintain.

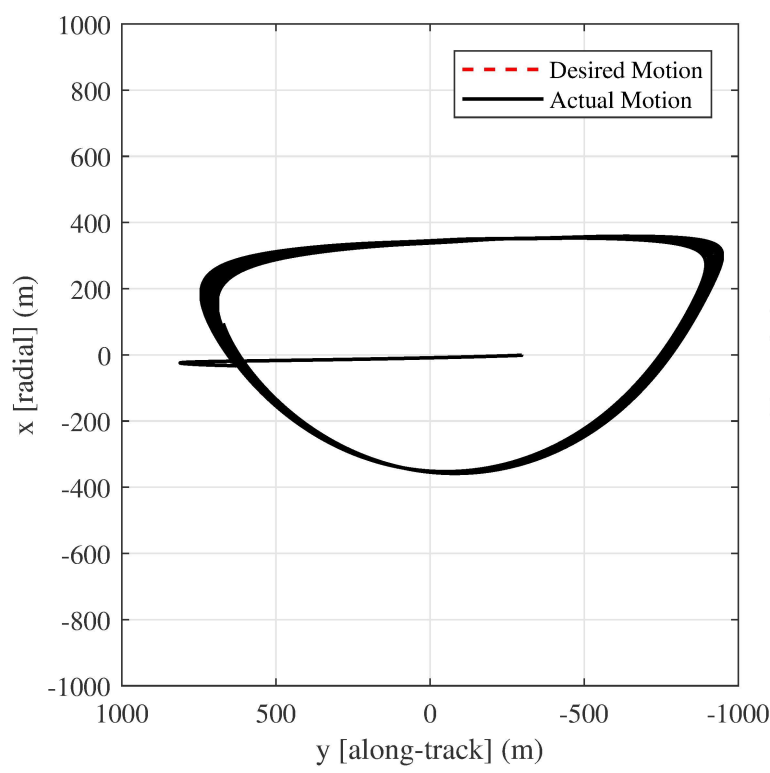

(a) Desired and actual in-plane motion

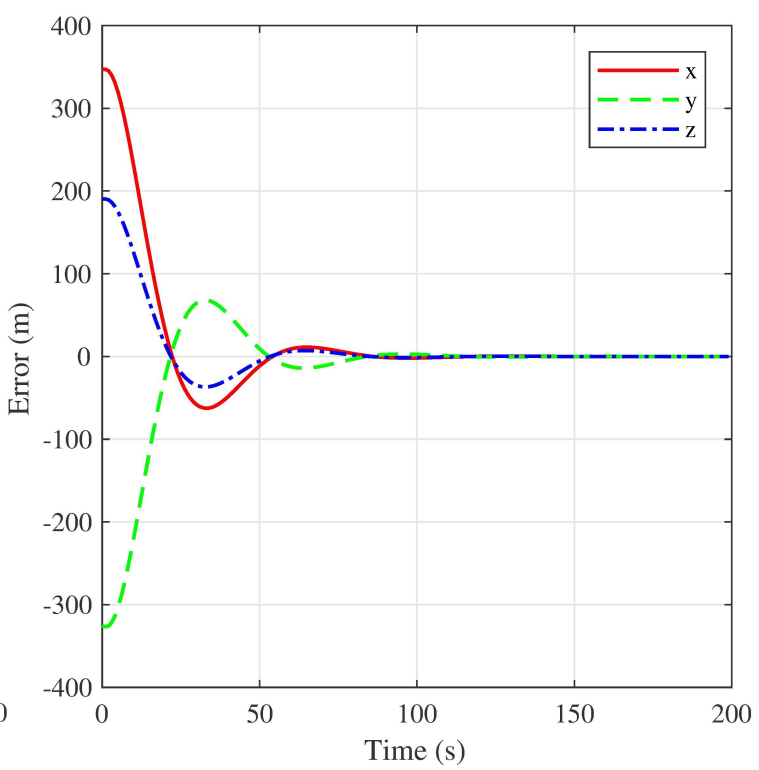

(b) Position error for first 200 seconds of simulation

Figure 5.13: Validation of control effectiveness for G\&C system using proposed analytical equations with a $J_{2}$-invariant orbit in a $J_{2}$-perturbed, highly elliptical orbit.

The differences between the HCW guidance, proposed equations guidance, and proposed equations guidance with the $J_{2}$-invariant orbit are shown in Fig. 5.15 below. 


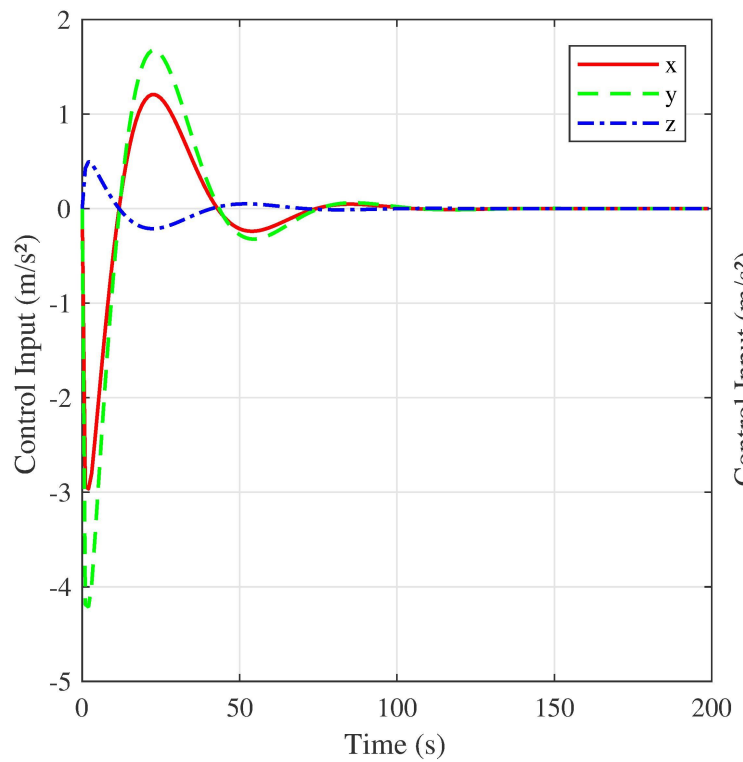

(a) Control input for first 200 seconds of simulation

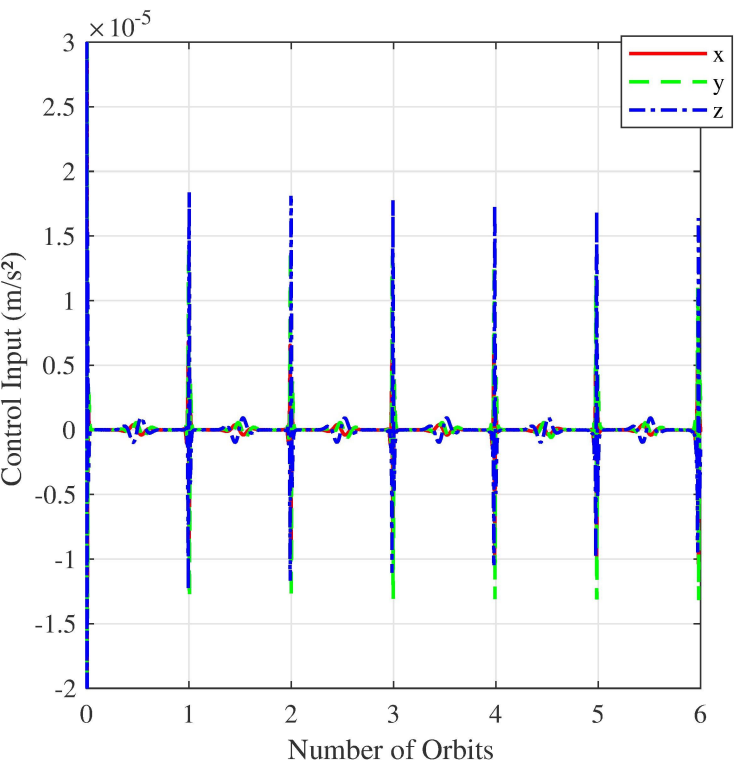

(b) Control input for entire simulation

Figure 5.14: Control inputs for G\&C system using proposed analytical equations with a $J_{2}$-invariant orbit in a $J_{2}$-perturbed, highly elliptical orbit.

\subsection{Back-Propagation With Continuous Control}

The reconfiguration maneuver using impulsive control inputs in the previous chapter works reasonably well, and demonstrates the potential application of back-propagation. However, the impulsive controller used in the previous section is best suited to use with mean orbital elements, and therefore is not the best fit for the proposed equations of motion which use the $J_{2}$-perturbed osculating orbital elements. Therefore, it is desirable to test the concept of back-propagation with another controller.

In this section, a time-continuous PD controller will be used with back-propagation to perform a reconfiguration maneuver. The simulation starts off very similarly to the previous example using impulsive control. The desired final orbital elements for the leader and follower spacecraft are defined, and this final, desired formation is propagated backwards in time with the proposed analytical equations. However, the initial orbital elements or relative position are no longer what is desired. Instead the proposed equations are used to define the relative position of the spacecraft in formation at each time step between the initial time and the final time. 


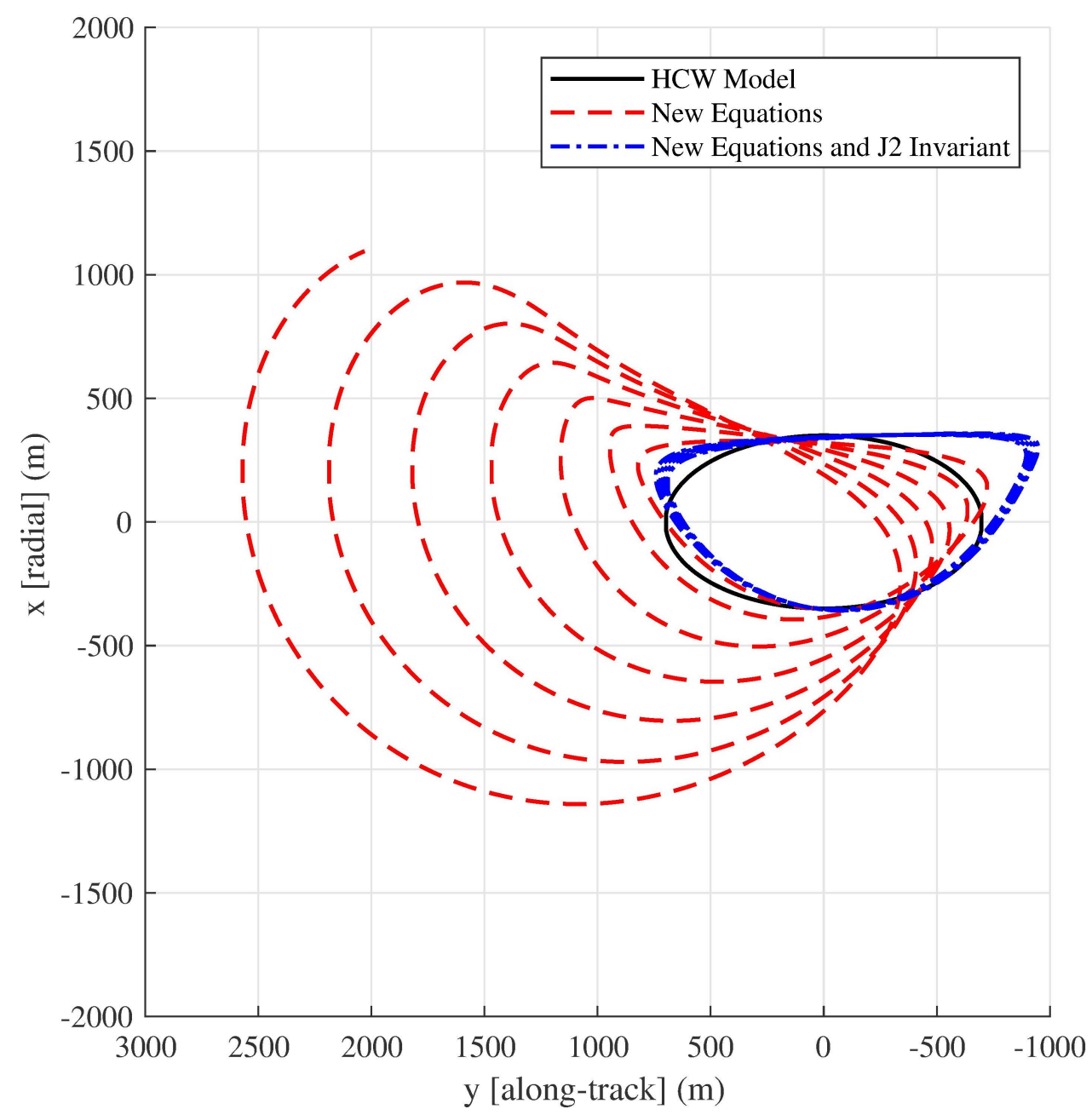

Figure 5.15: Comparison between HCW guidance, proposed guidance, and proposed guidance with a $J_{2}$ invariant orbit for in-plane motion of formation case in Table 5.3. 
This relative position and time data can then be used as the guidance in a G\&C simulation, as described in Sec. 5.2, and if the spacecraft is controlled to follow this guidance it will ultimately reach its desired position.

Two cases will once again be compared, both based on the formation initial conditions defined in Table 5.4. Once again, Case 1 will be representative of a more conventional approach to the reconfiguration maneuver. The spacecraft will be left in their respective initial orbits until right before the time at which the desired formation is required. For simplicity the control will be applied one orbital period before the end of the ten orbital period simulation, although with the time-continuous PD controller the desired orbit will be achieved well before the final time at which it is required.

Case 2 once again is the back-propagation case. Instead of letting the spacecraft drift apart in their initial orbits the desired relative position at each time step such that the formation drifts into the desired final formation is evaluated with backpropagation, and used as guidance. The PD controller is activated immediately, and the spacecraft once again reach the desired formation after 10 orbital periods.

Table 5.4: Orbital Elements for Continuous G\&C System (Back Propagation Case)

\begin{tabular}{|l|c|c|c|}
\hline Orbital Element & Desired Leader Final & Desired Follower Final & Follower Initial \\
\hline Semi-major Axis (km) & 315,000 & 15,000 & 15,001 \\
Eccentricity & 0.2 & 0.2001 & 0.2003 \\
Inclination (deg) & 59 & 59 & 58.95 \\
Argument of Perigee (deg) & 20 & 20 & 20.05 \\
RAAN (deg) & 84 & 84 & 83.8 \\
True Anomaly (deg) & 3 & 3 & 2.97 \\
\hline
\end{tabular}

Comparing Figs. 5.16 (a) and 5.18 (a) the differences between the two cases can clearly be seen. In Case 1, the spacccecraft drift apart for several orbits before the reconfiguration is applied, causing the initial error to be much larger once the controller is turned on. In Case 2, the less extreme initial errors are immediately corrected, and the spacecraft are placed into a drifting relative motion which follows the back-propagation guidance at each time step.

Figures. 5.16 (b) and 5.18 (b) and Figs. 5.17 (a) and 5.19 (a) once again show that the controller is working, reducing error via control accelerations applied to the spacecraft. Note that in the cases of Figs. 5.16 and 5.17, the first 200 seconds 
after the controller is applied are shown, as opposed to the first 200 seconds of the simulation as has been used in previous figures in this chapter. Also note that the relatively high accelerations needed show one downside of using a PD controller. This can be compared with the impulsive controller from Chap. 4, which corrects larger orbital element errors with significantly smaller maneuvers. Because the PD controller applies maneuvers continuously, with no regard for where in the orbit the spacecraft are, very large maneuvers are required. This is certainly not the most efficient way to perform reconfiguration maneuvers of this magnitude, but the results are still indicative of the advantages of the back-propagation guidance.

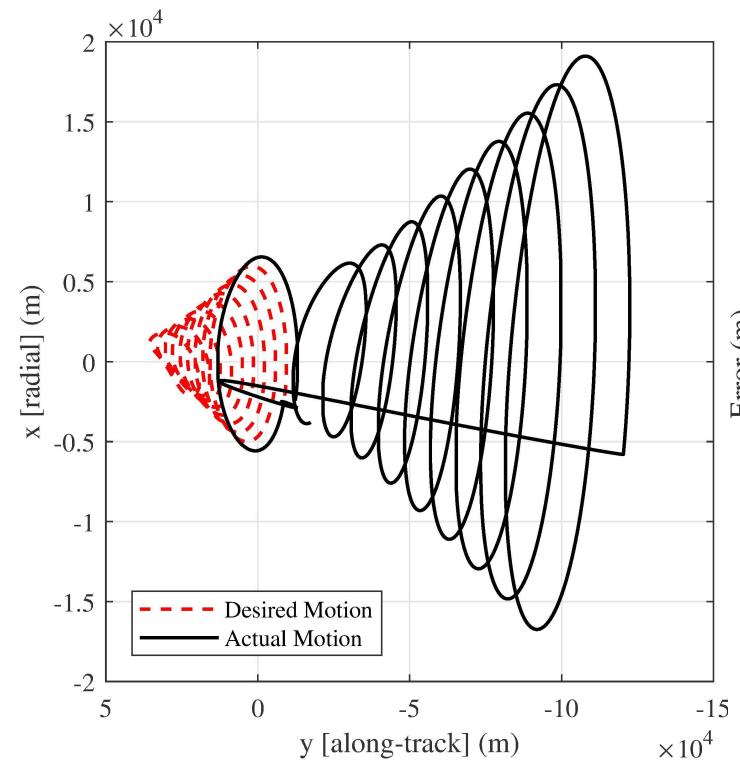

(a) Desired and actual in-plane motion

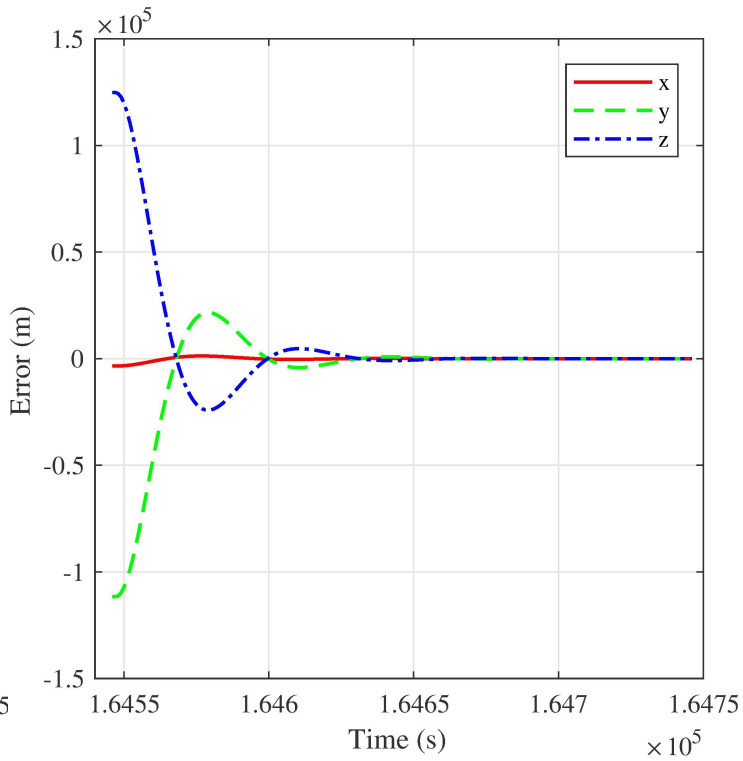

(b) Position error for first 200 seconds of simulation

Figure 5.16: Validation of control effectiveness for G\&C system using proposed analytical equations for back-propagation guidance in Case 1. 


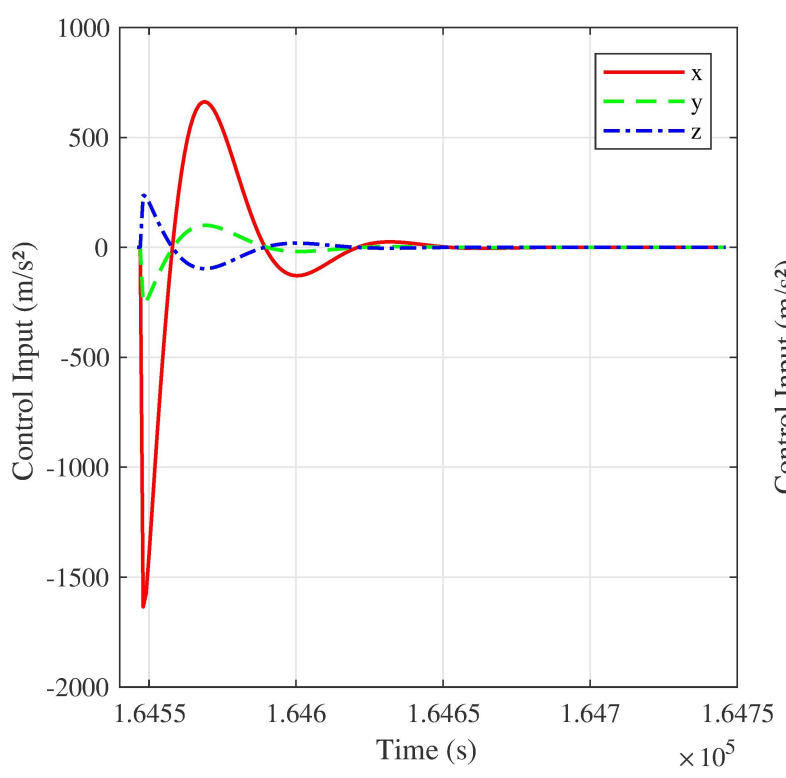

(a) Control input for first 200 seconds of simulation

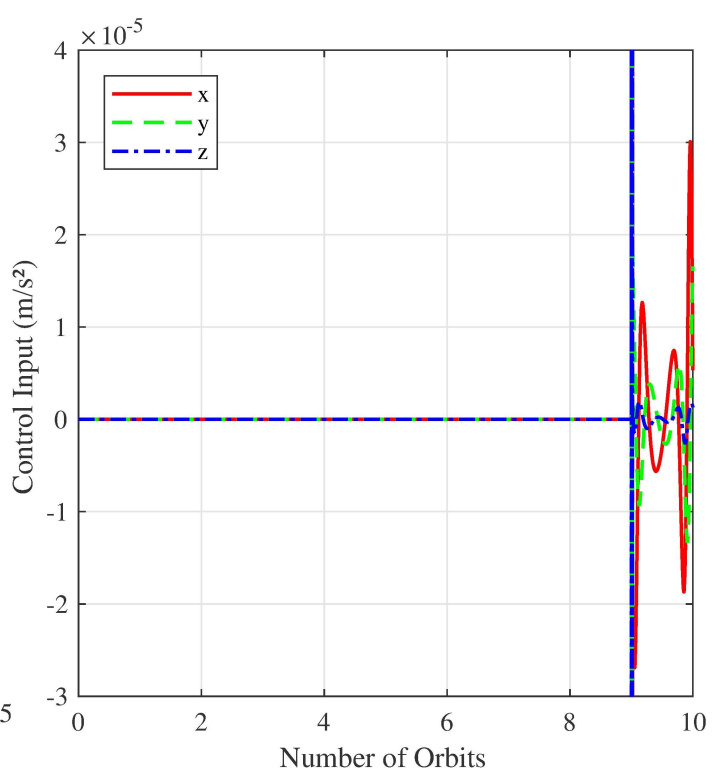

(b) Control input for entire simulation

Figure 5.17: Control inputs for G\&C system using proposed analytical equations for back-propagation guidance in Case 1.

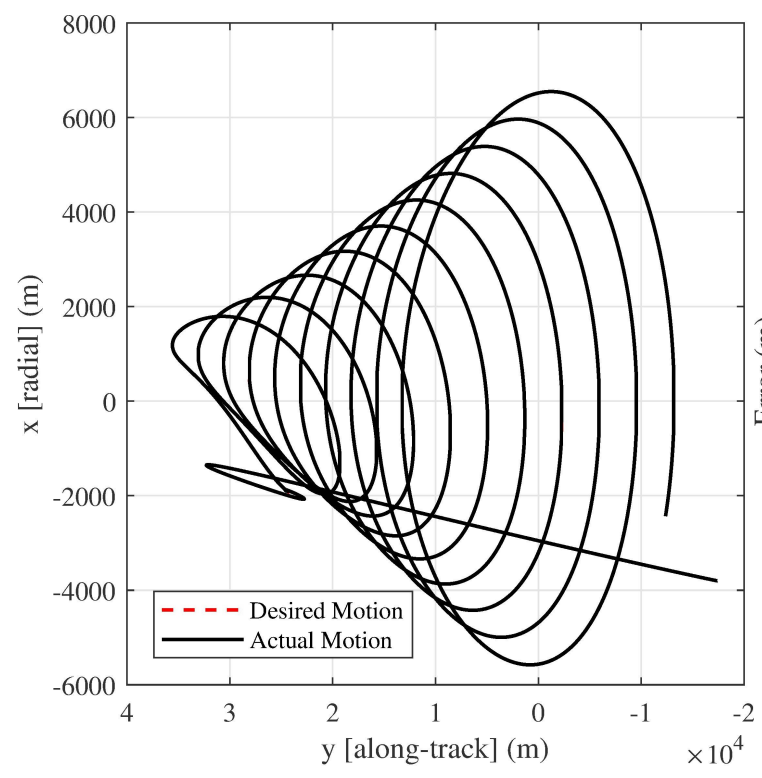

(a) Desired and actual in-plane motion

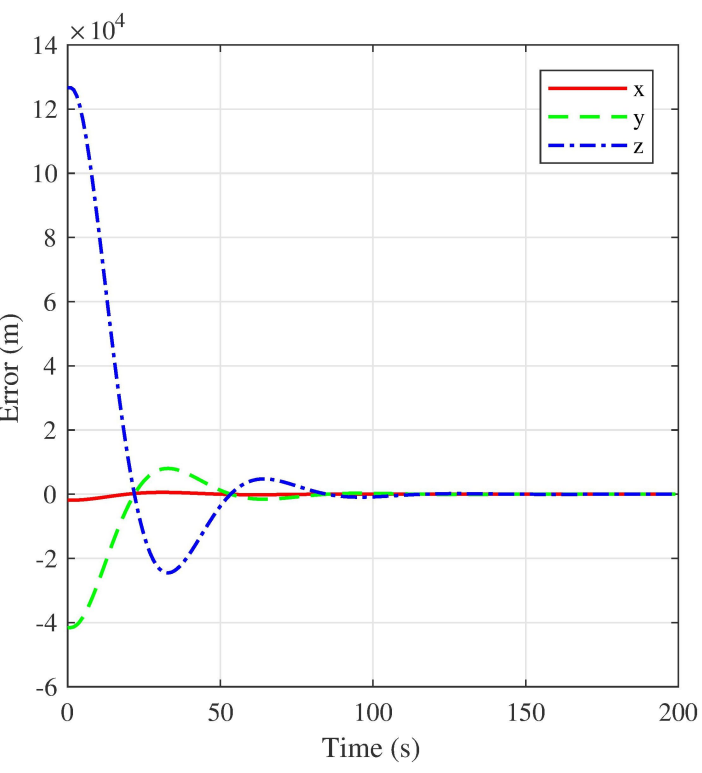

(b) Position error for first 200 seconds of simulation

Figure 5.18: Validation of control effectiveness for G\&C system using proposed analytical equations for back-propagation guidance in Case 2 . 


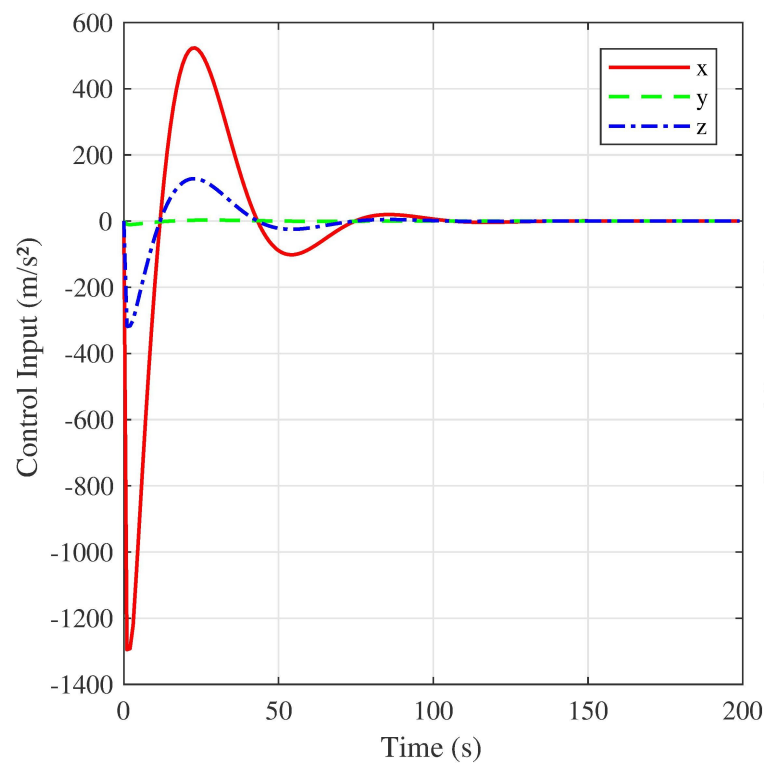

(a) Control input for first 200 seconds of simulation

Figure 5.19: Control inputs for G\&C system using proposed analytical equations for back-propagation guidance in Case 2. (b) Control input for entire simulation

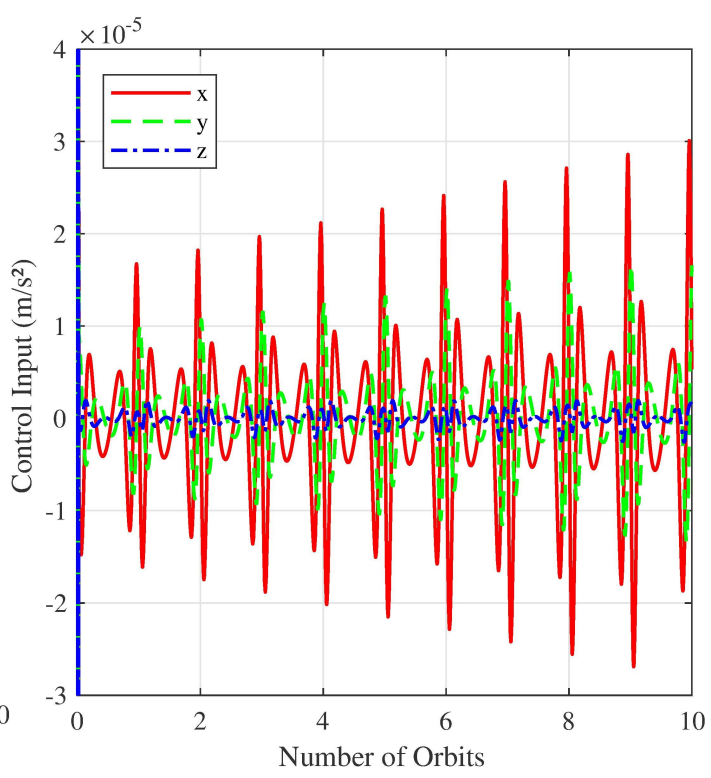




\section{Chapter 6}

\section{Conclusion}

This chapter presents a brief review of the motivation, objectives, and results of this thesis. The publications which resulted from the research for this thesis are also mentioned. Finally, the future work that could be undertaken to extend this research is discussed.

\subsection{Summary of Thesis}

The use of spacecraft formations in place of single spacecraft for a multitude of mission scenarios is an exciting but challenging prospect. The advantages of using formations are numerous. Redundancy of the mission could be increased as any problem that could render a spacecraft unusable will only affect one of a number of spacecraft, leaving the rest operational. Typically, spacecraft in formation are smaller and cheaper than a single spacecraft for a similar mission, making the cost of replacing one failed spacecraft much lower. Formations may also allow for more operational flexibility in the future, allowing missions to adapt the formation, and maybe even the number of spacecraft in formation to better meet changing mission goals. Finally there are certain missions which require formations to be practical, for example ESA's current PROBA-3 mission, and potential astronomy missions making use of interferometry which would require multiple spacecraft making observations at precise positions relative to each other.

All of these potential benefits of spacecraft formation flying are mainly limited by the ability of formations to hold the desired relative motion for the mission effectively and efficiently in terms of propellant use. In other words, although spacecraft formation flying may benefit many or most spacecraft missions, the difficulty in operating formations from an orbit guidance, navigation and control standpoint has limited the use of spacecraft formations thus far. Operating two or more spacecraft in close 
proximity is a more difficult task than controlling a single spacecraft, especially when the multiple spacecraft may be required to hold some specific relative motion.

This thesis sought to address some of the G\&C challenges faced by spacecraft formations; specifically the guidance system, which outputs the desired relative motion of the spacecraft formation. Many guidance methods exist for spacecraft formation flying, but most include certain assumptions which limit their ability to efficiently guide spacecraft in realistic orbits. For example, many potential guidance methods assume that the orbits of the spacecraft are circular, or that they are unperturbed. Making these assumptions may result in simple, convenient equations for guidance, but also results in guidance which is not accurate in most practical applications. When the guidance system does not accurately predict the natural spacecraft motion, it means that the G\&C system works harder to force the spacecraft into an unrealistic formation. For a guidance system to be effective it should be as simple (especially from a computational perspective) yet as accurate as possible. This allows the guidance to be used onboard the spacecraft, while also minimizing the effort (and the fuel required) for the $\mathrm{G} \& \mathrm{C}$ to maintain the formation.

In this context, novel analytical equations for the relative position of spacecraft in formation were derived in this thesis, which are valid for high eccentricity, $J_{2^{-}}$ perturbed orbits even with large separation distances. The accuracy of these equations was first validated by comparing the relative motion found using them with a numerical simulator. The result was that the proposed equations typically had error of about three orders of magnitude less than the spacecraft separation distances, and that the error was kept relatively low even after six orbital periods of propagation. In cases of very high eccentricity values (greater than 0.8), the errors did reach magnitudes of two orders of magnitude less than the spacecraft separation distance after six orbits, but this still satisfies the goal set out in Sec. 1.2.

With the knowledge that the proposed equations of motion were accurate, they were implemented in a number of closed-loop $\mathrm{G} \& \mathrm{C}$ simulations to demonstrate their practical application. They were first used as the guidance system with an impulsive controller making use of orbital element errors. The proposed equations were used to propagate the desired spacecraft relative position backwards in time to determine 
ideal initial conditions which allow the spacecraft to passively drift into their desired position. In this scenario, it was shown that using the proposed equations in this back-propagation scheme allows reconfiguration maneuvers with large initial position errors to be performed with less delta-v than a more conventional method. The proposed equations were then used as the guidance system in a time continuous G\&C system using a PD controller. In this case, relatively large initial errors were corrected to achieve a desired formation, and then this formation was maintained with smaller maneuvers. The novel guidance system worked well, demonstrating that a more natural formation which accounts for the eccentricity of the orbit and the $J_{2}$ perturbation, when combined with the novel guidance system, lowers the fuel cost of maintaining the formation when compared with the HCW model. Multiple formations were considered for this case, including formations with predictable drift caused by $J_{2}$ and formations in $J_{2}$-invariant orbits.

Ultimately the analytical expression for spacecraft relative motion sought by this thesis was achieved, with the resulting formulation meeting the goals of being analytical, applicable to large separation distances, and including the effects of $J_{2}$ and high eccentricity orbits. The application of this novel work in closed-loop G\&C scenarios serves to further validate the relative motion solution, and demonstrate the potential benefits of the work.

\subsection{Significance of Work}

The significance of this work is demonstrated by the publications and presentations that have resulted from the research, listed below:

B. Kuiack and S. Ulrich, Orbital Element-Based Relative Motion Guidance on J2-Perturbed Eccentric Orbits, in 2017 AAS/AIAA Astrodynamics Specialist Conference, Stevenson, WA, 2017. [33]

B. Kuiack and S. Ulrich, Nonlinear Analytical Equations of Relative Motion on J2-Perturbed Eccentric Orbits, in 26th AAS/AIAA Space Flight Mechanics Meeting, Napa, CA, 2016. [34] 


\subsection{Recommendations for Future Work}

There are a few different directions that this research could be taken for future work. The first, and perhaps most straightforward, is to implemenent the proposed guidance analytical equations as a guidance system in a hardware-in-the-loop G\&C simulation. This could be as simple as taking the simulation from Sec. 5.3 and adding real GPS hardware to measure the relative state variables. This would add a layer of realistic error into the simulation, and more realistic results could be analyzed as a result.

The poposed equations derived in this thesis could be demonstrated in a number of other practical mission scenarios. Specifically, interesting future work would involve adapting the equations to set up useful spacecraft formations. These may not be exactly the same as the typical formations like the projected circular arrangement in high eccentricity cases where the natural motion of the spacecraft will never be a perfectly circular shape, but formations with a variety of useful attributes may be possible.

Another interesting extension of this work would be to work on a more sophisticated controller to be used with the proposed guidance equations. The development of a controller which also accounts for the effects of the $J_{2}$ perturbation would pair well with the proposed guidance system to achieve highly efficient reconfiguration and formation keeping maneuvers.

The concept of back-propagation guidance for reconfiguration maneuvers could also be expanded on. Specifically, it would be interesting to study the possibility of optimizing a reconfiguration maneuver, determing the optimal time to begin a maneuver which most efficiently places the formation in a position such that they will drift into the desired formation.

Finally, it would be interesting to investigate the use of the proposed guidance equations with typical spacecraft processing hardware in an effort to determine whether or not the analytical equations of motion would be computationally simple enough to be used as on-board, autonomous guidance. More generally, future work should assess the computational performance of the proposed analytical solution compared to the numerical simulator in an effort to quantify how much of an improvement the nwe analytical solution offers. 


\section{Bibliography}

[1] D. Presti, J. Herman, and A. Codazzi. Mission Operations System Design and Adaptations for the Twin-Satellite Mission GRACE. In SpaceOps Conference, Montreal, Canada, 2004.

[2] R. Kahle, B. Schlepp, S. Aida, M. Kirschner, and M. Wermuth. Flight Dynamics Operations of the TanDEM-X Formation. In SpaceOps Conference, Stockholm, Sweden, 2012.

[3] S. D'Amico, J.-S. Ardaens, and R. Larsson. Spaceborne Autonomous FormationFlying Experiment on the PRISMA Mission. Journal of Guidance, Control, and Dynamics, 35(3):834-850, 2012.

[4] G. Di Mauro, M. Lawn, and R. Bevilacqua. Survey on Guidance Navigation and Control Requirements for Spacecraft Formation-Flying Missions. Journal of Guidance, Control, and Dynamics, 41(3):581-602, 2018.

[5] G. Bonin, N. Roth, S. Armitage, J. Newman, B. Risi, and R. E. Zee. CanX-4 ad CanX-5 Precision Formation Flight: Mission Accomplished! In 29th AIAA/USU Conference on Small Satellites, Logan, UT, 2015.

[6] W. H. Clohessy and R. S. Wiltshire. Terminal Guidance System for Satellite Rendezvous. Journal of the Aerospace Sciences, 27(9):653-658, 1960.

[7] C. Sabol and C. A. McLaughlin. Satellite Formation Flying Design and Evolution. Journal of Spacecraft and Rockets, 38(2):270-278, 2001.

[8] S. A. Schweighart and R. J. Sedwick. High-Fidelity Linearized $J_{2}$ Model for Satellite Formation Flight. Journal of Guidance, Control, and Dynamics, 25(6):10731080, 2002.

[9] G. Inalhan, M. Tillerson, , and J. How. Relative Dynamics and Control of Spacecraft Formations in Eccentric Orbits. Journal of Guidance, Control, and Dynamics, 25(1):48-59, 2002.

[10] H. Schaub. Relative Orbit Geometry Through Classical Orbit Element Differences. Journal of Guidance, Control, and Dynamics, 27(5):839-848, 2004.

[11] R. A. Broucke. Solution of the Elliptic Rendezvous Problem with the Time as Independant Variable. Journal of Guidance, Control, and Dynamics, 26(4):615$621,2003$. 
[12] D. J. Zanon and M. E. Campbell. Optimal Planner for Spacecraft Formations in Elliptical Orbits. Journal of Guidance, Control, and Dynamics, 29(1):161-171, 2006 .

[13] L. Breger, G. Inalhan, M. Tillerson, and J. How. Cooperative Spacecraft Formation Flying: Model Predictive Control with Open- and Closed-Loop Robustness. In P. Gurfil, editor, Modern Astrodynamics, chapter 8, pages 237-277. Elsevier Astrodynamics Series, 2006.

[14] T. E. Carter and M. Humi. Fuel-Optimal Rendezvous near a Point in General Keplerian Orbit. Journal of Guidance, Control, and Dynamics, 10(6):567-573, 1987.

[15] D. Lawden. Optimal Trajectories for Space Navigation. Butterworths, 1963.

[16] T. E. Carter. New Form for the Optimal Rendezvous Equations near a Keplerian Orbit. Journal of Guidance, Control, and Dynamics, 13(1):183-186, 1990.

[17] J. P. Marec. Optimal Space Trajectories. Elsevier, 1979.

[18] C. Lane and P. Axelrad. Formation Design in Eccentric Orbits Using Linearized Equations of Relative Motion. Journal of Guidance, Control, and Dynamics, 29(1):146-160, 2006.

[19] R. G. Melton. Time-Explicit Representation of Relative Motion Between Elliptical Orbits. Journal of Guidance, Control, and Dynamics, 23(4):604-610, 2000 .

[20] T. Guffanti, S D'Amico, and M. Lavagna. Long-Term Analytical Propagation of Satellite Relative Motion in Perturbed Orbits. In 27th AAS/AIAA Space Flight Mechanics Meeting, San Antonio, TX, 2017. AAS 17-355.

[21] P. Gurfil and K. V. Kholshevnikov. Distances on the Relative Spacecraft Motion Manifold. In AIAA Guidance, Navigation and Control Conference and Exhibit, San Francisco, CA, 2005. AIAA paper 2005-5859.

[22] P. Gurfil and K. V. Kholshevnikov. Manifolds and Metrics in the Relative Spacecraft Motion Problem. Journal of Guidance, Control, and Dynamics, 29(4):10041010, 2006.

[23] G. W. Hill. Researches in the Lunar Theory. American Journal of Mathematics, 1:5-26, 1878.

[24] P. Gurfil and N. J. Kasdin. Nonlinear Modeling of Spacecraft Relative Motion in the Configuration Space. Journal of Guidance, Control, and Dynamics, 27(1):154-157, 2004. 
[25] H. Schaub. Incorporating Secular Drifts into the Orbit Element Difference Description of Relative Orbits. Advances in the Astronautical Sciences, 114:239-258, 2003.

[26] D. Brouwer. Solution of the Problem of Artificial Satellite Theory without Drag. The Astronomical Journal, 64(1274):378-396, 1959.

[27] D. A. Vallado. Fundamentals of Astrodynamics and Applications, pages 604-612. Microcosm Press, El Segundo, CA, 2nd edition, 2001.

[28] R. R. Bate, D. D. Mueller, and J. E. White. Fundamentals of Astrodynamics, pages 419-425. Dover Publications, Inc., Mineola, NY, 1971.

[29] J. S. Llorente, A. Agenjo, Carrascosa C., C. de Negueruela, A. Mestreau-Garreau, A. Cropp, and A. Santovincenzo. PROBA-3: Precise Formation Flying Demonstration Mission. Acta Astronautica, 82(1):388-46, 2013.

[30] H. Schaub and K. T. Alfriend. Impulsive Feedback Control to Establish Specific Mean Orbit Elements of Spacecraft Formations. Journal of Guidance, Control, and Dynamics, 24(4):739-745, 2001.

[31] H. Schaub and K. T Alfriend. J2 Invariant Relative Orbits for Spacecraft Formations. Celestial Mechanics and Dynamical Astronomy, 79(2):77-95, 2001.

[32] H. Schaub, V. R. Vadali, J. L. Junkins, and K. T Alfriend. Spacecraft Formation Flying Control using Mean Orbit Elements. Journal of the Astronautical Sciences, 48(1):69-87, 2000.

[33] B. Kuiack and S. Ulrich. Orbital Element-Based Relative Motion Guidance on $J_{2^{-}}$ Perturbed Eccentric Orbits. In AAS/AIAA Astrodynamics Specialist Conference, Stevenson, WA, 2017. AAS 17-688.

[34] B. Kuiack and S. Ulrich. Nonlinear Analytical Equations of Relative Motion on $J_{2}$-Perturbed Eccentric Orbits. In AAS/AIAA Space Flight Mechanics Meeting, Napa, CA, 2016. AAS 16-495. 


\section{Appendix A}

\section{Step-By-Step Procedure for Proposed Relative Motion Solution}

This appendix presents a step-by-step procedure to propagate forward in time $\boldsymbol{\rho}$, the $J_{2}$-perturbed components of the relative position vector in the local-vertical-localhorizontal (LVLH) reference frame.

1. Two sets of Keplerian initial osculating orbital elements are specified, i.e., $\left[a_{0}, e_{0}, i_{0}, \omega_{0}, \Omega_{0}, \nu_{0}\right]^{T}$ and $\left[a_{0}^{\prime}, e_{0}^{\prime}, i_{0}^{\prime}, \omega_{0}^{\prime}, \Omega_{0}^{\prime}, \nu_{0}^{\prime}\right]^{T}$, where $a, e, i, \omega, \Omega, \nu$ denote the semi-major axis, eccentricity, inclination, argument of perigee, right ascension of ascending node, and true anomaly, respectively. The \{\}$^{\prime}$ symbol refers the leader spacecraft in the formation.

2. The mean anomaly of each spacecraft most also be initialized, i.e., $M_{0}$ and $M_{0}^{\prime}$. This is accomplished by working backwards from the true anomaly to the eccentric anomaly, denoted as $E$, and finally mean anomaly, as follows

$$
\begin{gathered}
E=M+e \sin (M+e \sin (M+e \sin (M+\cdots+e \sin (M)))) \\
\cos \nu=\frac{\cos E-e}{1-e \cos E} \\
\sin \nu=\frac{\sqrt{1-e^{2}} \sin E}{1-e \cos E} \\
\nu=\tan ^{-1} \frac{\sin \nu}{\cos \nu}
\end{gathered}
$$

3. The initial short-periodic variations, $\Delta\{\}_{s p}$, for each spacecraft are then calculated as a function of the initial osculating orbital elements, as follows[26] 


$$
\Delta a_{s p}=\frac{J_{2} R_{e}^{2}}{a}\left[\left(\frac{a}{r}\right)^{3}-\frac{1}{\left(1-e^{2}\right)^{3 / 2}}+\left\{-\left(\frac{a}{r}\right)^{3}+\frac{1}{\left(1-e^{2}\right)^{3 / 2}}+\left(\frac{a}{r}\right)^{3} \cos (2 \omega+2 \nu)\right\} \frac{3 \sin ^{2} i}{2}\right]
$$

$$
\begin{aligned}
\Delta e_{s p}= & \frac{J_{2} R_{e}^{2}}{4}\left[\frac{-2}{a^{2} e \sqrt{1-e^{2}}}+\frac{2 a\left(1-e^{2}\right)}{e r^{3}}+\left\{\frac{3}{a^{2} e \sqrt{1-e^{2}}}-\frac{3 a\left(1-e^{2}\right)}{e r^{3}}\right.\right. \\
& -\frac{3\left(1-e^{2}\right) \cos (\nu+2 \omega)}{p^{2}}-\frac{3 \cos (2 \nu+2 \omega)}{a^{2} e\left(1-e^{2}\right)}+\frac{3 a\left(1-e^{2}\right) \cos (2 \nu+2 \omega)}{e r^{3}} \\
& \left.\left.-\frac{\left(1-e^{2}\right) \cos (3 \nu+2 \omega)}{p^{2}}\right\} \sin ^{2} i\right]
\end{aligned}
$$

$$
\Delta i_{s p}=\frac{J_{2} R_{e}^{2} \sin (2 i)}{8 p^{2}}[3 \cos (2 \omega+2 \nu)+3 e \cos (2 \omega+\nu)+e \cos (2 \omega+3 \nu)]
$$

$$
\Delta \Omega_{s p}=\frac{J_{2} R_{e}^{2} \cos (i)}{4 p^{2}}[6(\nu-M+e \sin \nu)-3 \sin (2 \omega+2 \nu)-3 e \sin (2 \omega+\nu)-e \sin (2 \omega+3 \nu)]
$$

$$
\begin{aligned}
\Delta \omega_{s p}= & \frac{3 J_{2} R_{e}^{2}}{2 p^{2}}\left[\left(2-\frac{5}{2} \sin ^{2} i\right)(\nu-M+e \sin \nu)\right. \\
& +\left(1-\frac{3}{2} \sin ^{2} i\right)\left\{\frac{1}{e}\left(1-\frac{1}{4} e^{2}\right) \sin \nu+\frac{1}{2} \sin (2 \nu)+\frac{e}{12} \sin (3 \nu)\right\} \\
& -\frac{1}{e}\left\{\frac{1}{4} \sin ^{2} i+\left(\frac{1}{2}-\frac{15}{16} \sin ^{2} i\right) e^{2}\right\} \sin (\nu+2 \omega)+\frac{e}{16} \sin ^{2} i \sin (\nu-2 \omega) \\
& -\frac{1}{2}\left(1-\frac{5}{2} \sin ^{2} i\right) \sin (2 \nu+2 \omega) \\
& +\frac{1}{e}\left\{\frac{7}{12} \sin ^{2} i-\frac{1}{6}\left(1-\frac{19}{8} \sin ^{2} i\right) e^{2}\right\} \sin (3 \nu+2 \omega) \\
& \left.+\frac{3}{8} \sin ^{2} i \sin (4 \nu+2 \omega)+\frac{e}{16} \sin ^{2} i \sin (5 \nu+2 \omega)\right]
\end{aligned}
$$




$$
\begin{aligned}
\Delta M_{s p}= & \frac{3 J_{2} R_{e}^{2} \sqrt{1-e^{2}}}{2 e p^{2}}\left[-\left(1-\frac{3}{2} \sin ^{2}(i)\right)\left\{\left(1-\frac{e^{2}}{4}\right) \sin \nu+\frac{e}{2} \sin (2 \nu)+\frac{e^{2}}{12} \sin (3 \nu)\right\}\right. \\
& +\sin ^{2} i\left\{\frac{1}{4}\left(1+\frac{5}{4} e^{2}\right) \sin (\nu+2 \omega)-\frac{e^{2}}{16} \sin (\nu-2 \omega)\right. \\
& \left.\left.-\frac{7}{12}\left(1-\frac{e^{2}}{28}\right) \sin (3 \nu+2 \omega)-\frac{3 e}{8} \sin (4 \nu+2 \omega)-\frac{e^{2}}{16} \sin (5 \nu+2 \omega)\right\}\right]
\end{aligned}
$$

where $J_{2}$ is the value of the perturbation due to the oblateness of the Earth, $R_{e}$ is the mean equatorial radius of the Earth, $\mathrm{r}$ is the magnitude of the position vector of the spacecraft in the Earth-Centered-Inertial (ECI) reference frame, and $p$ is the semilatus rectum.

4. By rearranging Eq. (A.17), the initial mean orbital elements for each spacecraft are calculated from the initial short-periodic variations subtracted from the initial osculating orbital elements, that is

$$
\begin{aligned}
& \bar{a}_{0}=a_{0}-\Delta a_{0_{s p}} \\
& \bar{e}_{0}=e_{0}-\Delta e_{0_{s p}} \\
& \bar{i}_{0}=i_{0}-\Delta i_{0_{s p}} \\
& \bar{\omega}_{0}=\omega_{0}-\Delta \omega_{0_{s p}} \\
& \bar{\Omega}_{0}=\Omega_{0}-\Delta \Omega_{0_{s p}} \\
& \bar{M}_{0}=M_{0}-\Delta M_{0_{s p}}
\end{aligned}
$$

and similarly for the leader spacecraft.

5. The mean semi-major axis, eccentricity and inclination have no secular variation, and are therefore equal to their initial mean values, as calculated in the previous step, i.e., $\bar{a}=\bar{a}_{0}, \bar{e}=\bar{e}_{0}$, and $\bar{i}=\bar{i}_{0}$, as well as $\bar{a}^{\prime}=\bar{a}_{0}^{\prime}, \bar{e}^{\prime}=\bar{e}_{0}^{\prime}$, and $\bar{i}^{\prime}=\bar{i}_{0}^{\prime}$. The mean orbital motion and mean semilatus rectum for each spacecraft, $\bar{n}, \bar{p}, \bar{n}^{\prime}$, and $\bar{p}^{\prime}$, are calculated at this point as these parameters are only 
functions of mean semi-major axis and mean eccentricity

$$
\begin{gathered}
\bar{n}=\sqrt{\frac{\mu}{\bar{a}^{3}}} \\
\bar{p}=\bar{a}\left(1-\bar{e}^{2}\right)
\end{gathered}
$$

6. The remaining mean orbital elements, $[\bar{\omega}, \bar{\Omega}, \bar{M}]^{T}$ and $\left[\bar{\omega}^{\prime}, \bar{\Omega}^{\prime}, \bar{M}^{\prime}\right]^{T}$, can be propagated forward in time, from $t_{0}$ to $t$, by summing mean orbital elements with the $J_{2}$-induced secular variations, as follows

$$
\begin{aligned}
& \bar{\omega}=\bar{\omega}_{0}+\dot{\bar{\omega}} t \\
& \bar{\Omega}=\bar{\Omega}_{0}+\dot{\bar{\Omega}} t \\
& \bar{M}=\bar{M}_{0}+\dot{\bar{M}} t
\end{aligned}
$$

where[27]

$$
\begin{gathered}
\dot{\bar{\omega}}=\frac{1}{2} \bar{n} J_{2}\left(\frac{R_{e}}{\bar{p}}\right)^{2}\left(4-5 \sin ^{2} \bar{i}\right) \\
\dot{\bar{\Omega}}=-\frac{3}{2} \bar{n} J_{2}\left(\frac{R_{e}}{\bar{p}}\right)^{2} \cos \bar{i} \\
\dot{\bar{M}}=\bar{n}+\frac{3}{2} \bar{n} J_{2}\left(\frac{R_{e}}{\bar{a}}\right)^{2} \frac{1}{\left(1-\bar{e}^{2}\right)^{3 / 2}}\left(1-\frac{3}{2} \sin ^{2} \bar{i}\right)
\end{gathered}
$$

7. Based on the average mean anomalies, $\bar{M}$ and $\bar{M}^{\prime}$, the true anomaly for each spacecraft, $\nu$ and $\nu^{\prime}$, are propagated forward in time using Eqs. (A.1)-(A.4).

8. Now that the true anomalies have been propagated forward in time, the shortperiodic variations can then be calculated for each spacecraft using Eqs. (A.5)(A.10). The osculating orbital elements are then propagated forward in time as 


$$
\begin{aligned}
& a=\bar{a}+\Delta a_{s p} \\
& e=\bar{e}+\Delta e_{s p} \\
& i=\bar{i}+\Delta i_{s p} \\
& \omega=\bar{\omega}+\Delta \omega_{s p} \\
& \Omega=\bar{\Omega}+\Delta \Omega_{s p} \\
& M=\bar{M}+\Delta M_{s p}
\end{aligned}
$$

9. Finally, $\boldsymbol{\rho}$ is given by

$$
\boldsymbol{\rho}=\boldsymbol{C}_{L P^{\prime}}\left(\nu^{\prime}\right) \boldsymbol{C}_{P^{\prime} I}\left(\omega^{\prime}, i^{\prime}, \Omega^{\prime}\right) \boldsymbol{C}_{I P}(\omega, i, \Omega) \boldsymbol{r}_{P}-\boldsymbol{r}_{L}^{\prime}
$$

where the different rotation matrices between LVLH, $\mathcal{F}_{L}$, ECI, $\mathcal{F}_{I}$, and perifocal, $\mathcal{F}_{P}$, reference frames are obtained as

$$
\begin{gathered}
\boldsymbol{C}_{I L}(\omega, i, \Omega)=\left[\begin{array}{ccc}
c_{\Omega} c_{u}-s_{\Omega} s_{u} c_{i} & -c_{\Omega} s_{u}-s_{\Omega} c_{u} c_{i} & s_{\Omega} s_{i} \\
s_{\Omega} c_{u}+c_{\Omega} s_{u} c_{i} & -s_{\Omega} s_{u}-c_{\Omega} c_{u} c_{i} & -c_{\Omega} s_{i} \\
s_{u} s_{i} & c_{u} s_{i} & c_{i}
\end{array}\right] \\
\boldsymbol{C}_{L^{\prime} I}\left(\omega^{\prime}, i^{\prime}, \Omega^{\prime}\right)=\left[\begin{array}{ccc}
c_{\Omega^{\prime}} c_{u^{\prime}}-s_{\Omega^{\prime}} s_{u^{\prime}} c_{i^{\prime}} & s_{\Omega^{\prime}} c_{u^{\prime}}+c_{\Omega^{\prime}} s_{u^{\prime}} c_{i^{\prime}} & s_{u^{\prime}} s_{i^{\prime}} \\
-c_{\Omega^{\prime}} s_{u^{\prime}}-s_{\Omega^{\prime}} c_{u^{\prime}} c_{i^{\prime}} & -s_{\Omega^{\prime}} s_{u^{\prime}}-c_{\Omega^{\prime}} c_{u^{\prime}} c_{i^{\prime}} & c_{u^{\prime}} s_{i^{\prime}} \\
s_{\Omega^{\prime}} s_{i^{\prime}} & -c_{\Omega^{\prime}} s_{i^{\prime}} & c_{i^{\prime}}
\end{array}\right]
\end{gathered}
$$

where $\mathrm{u}$ is the argument of latitude, defined as $u=\nu+\omega$ and $u^{\prime}=\nu^{\prime}+\omega^{\prime}$. The components of the follower position vector in $\mathcal{F}_{P}$ and those of the leader position vector in $\mathcal{F}_{L}$ are evaluated with the $J_{2}$-perturbed osculating orbital elements.

$$
\begin{gathered}
\boldsymbol{r}_{P}=\left[\begin{array}{lll}
r \cos \nu & r \sin \nu & 0
\end{array}\right]^{T} \\
\boldsymbol{r}_{L}^{\prime}=\left[\begin{array}{lll}
r^{\prime} & 0 & 0
\end{array}\right]^{T}
\end{gathered}
$$

